\title{
Untersuchungen zur Optimierung eines Gammakameradetektors durch die Auswertung seiner verrauschten Antwort auf die Gammaquanten aus einer verfahrbaren Feinnadelstrahlquelle
}

\author{
Dissertation \\ zur Erlangung des Doktorgrades \\ der Mathematisch-Naturwissenschaftlichen Fakultäten \\ der Georg-August-Universität zu Göttingen
}

\author{
vorgelegt von \\ Uwe Engeland \\ aus Wyk auf Föhr
}

Göttingen 2000 
D 7

Referent: Prof. Dr. A.Zippelius

Koreferent: Prof. Dr. Dr. H.Luig

Tag der mündlichen Prüfung: 31.1.2001 


\section{Zusammenfassung}

In dieser Arbeit wird ein neues Verfahren vorgestellt, mit dem das Abbildungsverhalten einer Gammakamera durch eine Korrektion verbessert wird. Die Korrektion setzt sich aus zwei unabhängigen Teilen zusammen.

Der erste Teil betrifft die Korrektion der Ortskoordinaten und der mit der Energie des $\gamma$-Quants in Beziehung stehender Impulsantwort detektierter $\gamma$-Quanten und wird in Kapitel 3 dargestellt. Grundlage dieser Korrektion stellen dabei Daten dar, die mit Hilfe einer Feinnadelstrahlquelle aufgenommen wurden. Der feine Strahl von $\gamma$-Quanten aus dieser Art Quelle stellt die beste zur Zeit durchführbare Annäherung an einen Deltapuls im Sinne der Orts- und Impulshöhenkoordinaten dar. Damit wird erstmalig die Abbildungscharakteristik der Gammakamera einer örtlich fein aufgelösten Analyse zugänglich. Zusätzlich wurde ein Computerprogramm erstellt, das das Abbildungsverhalten der Gammakamera in Einzelheiten simuliert. So war es möglich, einen Test des neuen Korrekturverfahrens durchzuführen. Daneben wurde das Verfahren durch die Erstellung von planaren Szintigrammen verschiedener Testmuster geprüft. Die dabei verwendete Gammakamera wurde absichtlich dekalibiert. Es konnte gezeigt werden, daß das Verfahren mit einer derart dekalibrierten Kamera in erwarteter Weise arbeitet und die bildwirksamen Folgeerscheinungen der Dekalibrierung weitgehend aufheben kann.

Der zweite Teil ist ein neues Korrekturverfahren im Sinne einer neuen Statistik der Zählung von $\gamma$-Quanten mit einer Gammakamera und findet sich in Kapitel 4. Dabei wird der Verwerfung von Ereignissen als Folge der Totzeit der Verarbeitungselektronik Rechnung getragen. Über ein Modell des Zählprozesses kann für eine Klasse von Kameras eine gute Schätzung der tatsächlichen Zählrate aus der gemessenen Zählrate abgeleitet werden. Es wird zum ersten Mal ein Schätzer vorgestellt, welcher den gesamten Zählratenbereich der Gammakamara überdeckt und im Unterschied zu anderen nicht nur für bestimmte Zählraten gültig ist. Das Modell basiert auf der konsequenten Berücksichtung der der Zählung zugrunde liegenden Vorgänge in der Gammakamera selbst, genauer: der Überlagerung der einzelnen Impulse. Das Ergebnis ist ein neuer Schätzer der Zählraten, der unabhängig von gegebenenfalls verwendeten Impulshöhen- oder Ortsfenstern ist. 


\section{Inhaltsverzeichnis}

1 Einleitung 1

2 Grundlagen der szintigraphischen Abbildung 5

2.1 Funktionsweise der Gammakamera nach Anger: Überblick . . . . . . . . . 5

2.2 Beschreibung des Abbildungsvorgangs . . . . . . . . . . . . . . 7

2.2.1 Teil 1: Emission - Kristall . . . . . . . . . . . . . . . . 9 9

2.2.2 Teil 2: Kristall - Photomultiplier . . . . . . . . . . . . . 11

2.2 .3 Teil 3: Signalverarbeitung . . . . . . . . . . . . . . . . . 16

2.3 Charakterisierung der Abbildungsfehler . . . . . . . . . . . . . . . 18

2.4 Simulation . . . . . . . . . . . . . . . . . . 21

2.4.1 Das Modell . . . . . . . . . . . . . . . . . 22

2.4.2 Szintigramme aus der Simulation . . . . . . . . . . . . 24

3 Korrektion $\quad 27$

3.1 Das Konzept. . . . . . . . . . . . . . . . . . . . . 29

3.1.1 Die Feinnadelstrahlquelle . . . . . . . . . . . . . . . 32

3.1.2 Die Abbildung der $\gamma$-Quanten aus der Feinnadelstrahlquelle durch die Gammakamera . . . . . . . . . . . . . . . . . . 37

3.1.3 Pixel-Definition . . . . . . . . . . . . . . . . . . . . . . . 40

3.1 .4 Das Pixelraster . . . . . . . . . . . . . . . . . 46

3.2 Das Verfahren in der Anwendung . . . . . . . . . . . . . . . . 51

4 Statistik der Zählung mit einer Gammakamera 63

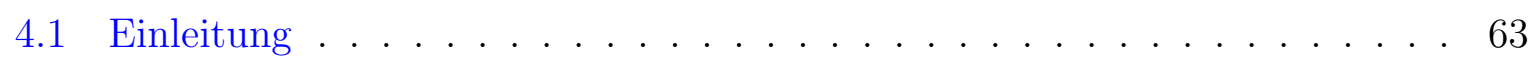

4.2 Bisherige Verfahren . . . . . . . . . . . . . . . . . . 64

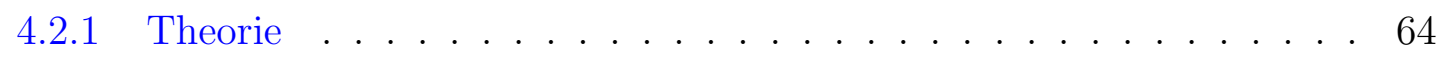


4.2.2 Anwendung auf die Gammakamera . . . . . . . . . . . . . . . 66

4.3 Das Modell der Signalüberlagerungsverluste . . . . . . . . . . . . . . . . . 68

4.3.1 Die Signalüberlagerung (engl:Pile-up) . . . . . . . . . . . . . . . 68

4.3.2 Das neue Modell . . . . . . . . . . . . . . . . . . . . . . . . . 70

4.4 Der neue Schätzer . . . . . . . . . . . . . . . . . . . . . . 75

4.5 Messungen und Simulationen . . . . . . . . . . . . . . . 78

4.5.1 Computersimulation . . . . . . . . . . . . . . . . 78

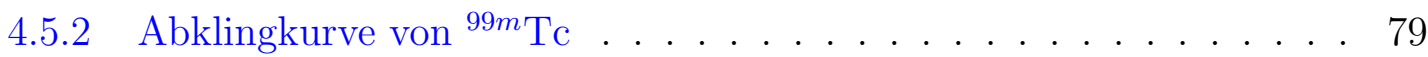

4.5.3 ${ }^{57}$ Co Punktquelle neben einer initial hochaktiven ${ }^{99 m} \mathrm{Tc}$ Punktquelle . . . . . . . . . . . . . . . . 80

5 Schlußbemerkungen und Ausblick $\quad 83$

A Zur Simulation $\quad 89$

A.1 Bestimmung der Brechungswinkel . . . . . . . . . . . . . . . . . 89

A.2 Berücksichtigung von Reflektionen . . . . . . . . . . . . . . . . . . 90 


\section{Einleitung}

Mit Hilfe einer Gammakamera werden räumliche Verteilungen von Radionukliden detektiert. Die Kamera weist dabei jedem detektierten Gammaquant zwei Ortskoordinaten, eine der Energie des Gammaquants entsprechende Impulshöhe ${ }^{1}$ und einen Zeitpunkt der Detektion zu. Eine Gammakamera stellt also einen ortsauflösenden Zähler mit eingeschränkten spektroskopischen Fähigkeiten dar.

Die Menge aller detektierter Gammaquanten einer Aufnahme kann als zweidimensionales Bild dargestellt, oder besser, interpretiert werden. Aus der Menge aller detektierter Gammaquanten werden diejenigen herausgesucht, deren Impulshöhe in einem anzugebenden Bereich liegt. Aus den Ortskoordinaten dieser ausgesuchten Gammaquanten wird ein zweidimensionales Histogramm erstellt. Dieses kann als eine projektive Abbildung der dreidimensionalen Ortsdichteverteilung des Radionuklids interpretiert werden. Ein in dieser Art gebildetes Histogramm wird als Szintigramm bezeichnet.

Das Abbildungsverhalten der Gammakamera weicht von einer idealen Abbildung z.T. erheblich ab, so daß sich die Qualität der entstehenden Szintigramme u.U. erheblich verschlechtern können. Die Abweichungen müssen daher korrigiert werden. Als Abbildungsfehler werden in dieser Arbeit sowohl die verfälschten Zuweisungen zu den Orts- und Impulshöhenkoordinaten eines detektierten Gammaquants verstanden, wie auch die Unterschlagung einzelner oder mehrerer Gammaquanten aufgrund des endlichen zeitlichen Auflösungsvermögens der Gammakamera. Es muß auf den Umstand hingewiesen werden, daß es sich bei der Korrektion der Orts- und Impulskoordinaten um die Korrektion gemessener Werte handelt, wohingegen die Korrektur der Wirkung des endlichen zeitlichen Auflösungsvermögens durch Abschätzung fehlender Meßwerte bei der Zählung mit Hilfe

\footnotetext{
${ }^{1}$ Unter dem Begriff der Impulshöhe versteht man die Summe der zeitintegrierten Photomultipliersignale von allen an der Detektion eines $\gamma$-Quants beteiligten Photomultiplier. Die Höhe dieses Signals ist u.a. abhängig von der Energie des $\gamma$-Quants. Aufgrund des komplizierten Zusammenhangs ist es nur als ein grobes Maß für die Energie zu sehen, nicht als regulärer Meßwert der Energie.
} 
einer Modelltheorie vorgenommen wird.

Da Gammaquanten hoher Energie, hier etwa $50 \mathrm{keV}$ - $1 \mathrm{MeV}$, keinen optischen Manipulationen zugänglich sind, findet die Korrektion erst dem eigentlichen Abbildungsvorgang nachgeschaltet auf rechnerische Art statt.

Allen Verfahren zur Korrektion der Orts- und Impulshöhendetektion ist gemeinsam, daß eine als bekannt vorausgesetzte Verteilung eines Radionuklids aufgenommen wird. Aus dem Vergleich der tatsächlichen Abbildung mit dem zu erwartenden Bild werden die Abbildungsfehler bestimmt und Korrektionsvorschriften abgeleitet. Die Formulierung „als bekannt vorausgesetzte Verteilung“" macht die damit verbundene Problematik deutlich: Inwieweit die Voraussetzung der Kenntnis der Verteilung gegeben ist, ist oft zumindestens zweifelhaft und stellt somit auch das Wissen um das zu erwartende Bild in Frage. Allerdings bildet die Güte dieser Kenntnis die Grundlage für die Güte der Korrektionen. So setzt das hier beschriebenen Verfahren genau an diesem Punkt an, um neue Wege zur Korrektion der Abbildung einer Gammakamera zu finden. Vorbild ist die durchaus übliche Herangehensweise an abbildende lineare Systeme: Um den Einfluß des Abbildungsvorganges herauszurechnen wird dem System ein $\delta$-Impuls angeboten. Die Antwort stellt die sog. Systemfunktion dar, mit deren Hilfe eine Entfaltung zur Erlangung des korrigierten Bildes durchgeführt werden kann. Auch wenn eine Entfaltung aus Gründen des äußerst geringen Signal-Rauschabstandes im Szintigramm nicht das Ziel sein kann, so enthielte die Antwort auf einen $\delta$-Impuls doch die am besten aufgelöste Information.

Ein idealer $\delta$-Impuls ist für $\gamma$-Quanten mit den zur Verfügung stehenden Mitteln sicher nicht zu erreichen. Um dem Ideal möglichst nahe zu kommen und gleichzeitig ein praktikables Verfahren zu erhalten, wurde im Rahmen dieser Arbeit die sog. Feinnadelstrahlquelle konzipiert. Mit ihr steht eine gute Annährung an einen $\delta$-Impuls zur Verfügung. Mit dem hochkollimierten Strahl ist es möglich, dem Detektorkopf in einem sequentiell durchfahrenen feinen Raster $\gamma$-Quanten anzubieten und die Antworten überlagerungsfrei - hier im Sinne unterschiedlicher Detektororte - zu registrieren. Somit ist die Kenntnis der angebotenen Nuklidverteilung hervorragend gegeben und darauf aufbauend konnte eine wirkungsvolle Korrektionsvorschrift entwickelt werden.

Das Verhalten der Gammakamera als ortender Zähler führt unter Berücksichtigung des endlichen zeitlichen Auflösungsvermögens zu einer neuen Statistik der Zählung von $\gamma$ Quanten mit einer Gammakamera, weil die Forderung der korrekten Ortung des $\gamma$-Quants eine stärke Einschränkung darstellt: während der Verarbeitung der durch die Szintillation ausgelösten Signale darf kein weiteres $\gamma$-Quant eine Szintillation auslösen. Die reine 
Zählung des ersten und u.U. auch des folgenden $\gamma$-Quants ist durchaus möglich, jedoch keine Ortung, die mit den tatsächlichen Orten der beteiligten Quanten in einen sinnvollen Zusammenhang gebracht werden kann. Somit werden beide Quanten nicht nur für die Erstellung eines Szintigramms wertlos, sondern führen auch zu schlicht falschen Bildinformationen und müssen verworfen werden. Im Rahmen dieser Arbeit ist ein Schätzer entwickelt worden, der aus den überlagerungsfrei - hier im Sinne der beteiligten Signale detektierten auf die Anzahl der verworfenen $\gamma$-Quanten schließt und somit berücksichtigt. 


\section{Grundlagen der szintigraphischen Abbildung mit einer Gammakamera}

In diesem Kapitel werden die Grundlagen und das Ziel der szintigraphischen Abbildung mit einer Gammakamera nach Anger [4] dargestellt. In Abschnitt 2.1 werden die Grundprinzipien einer Gammakamera erläutert. In den darauf folgenden Abschnitten 2.2.1, 2.2.2 und 2.2.3 werden diejenigen Phänomene des Abbildungsvorgang diskutiert, die die Bildqualität einer szintigraphischen Aufnahme beeinflussen. Die hieraus gewonnenen Einsichten werden im Abschnitt 2.4 zur Simulation des Abbildungsverhaltes der Gammakamera genutzt.

\subsection{Funktionsweise der Gammakamera nach Anger: Überblick}

Die Gammakamera ist ein Gerät zur Detektion von Gammastrahlung. Neben ihrer Funktion als Zählgerät der einfallenden Gammaquanten weist sie diesen ebenfalls einen Detektionsort und eine Impulshöhe zu. Jedes Gammaquant ist also durch vier Koordinatenwerte $t, x, y$ und $e$ ausgezeichnet: Zeitpunkt $t$ der Detektion, Schwerpunktskoordinatenpaar $x$ und $y$ der Wechselwirkung mit dem Detektionskristall und Impulshöhe $e$. Die Impulshöhe ist zu der Energie des Gammaquants im Rahmen der Meßgenauigkeit proportional. Der Energiebereich der Quanten, die mit einer Gammakamera sinnvoll abgebildet werden können, liegt je nach Bauart zwischen $50 \mathrm{keV}$ und $1 \mathrm{MeV}$.

Ziel der Szintigraphie mit einer Gammakamera ist es, ein Bild der räumlichen Verteilung von mit Gammastrahlern markierten Radiopharmaka zu gewinnen. Der Begriff Bild ist dabei so zu verstehen, daß der Inhalt - z.B. kodiert durch eine Graustufe - eines Bildpunktes proportional zur Aktivität $a$ ist, die innerhalb desjenigen Volumens liegt, welches 


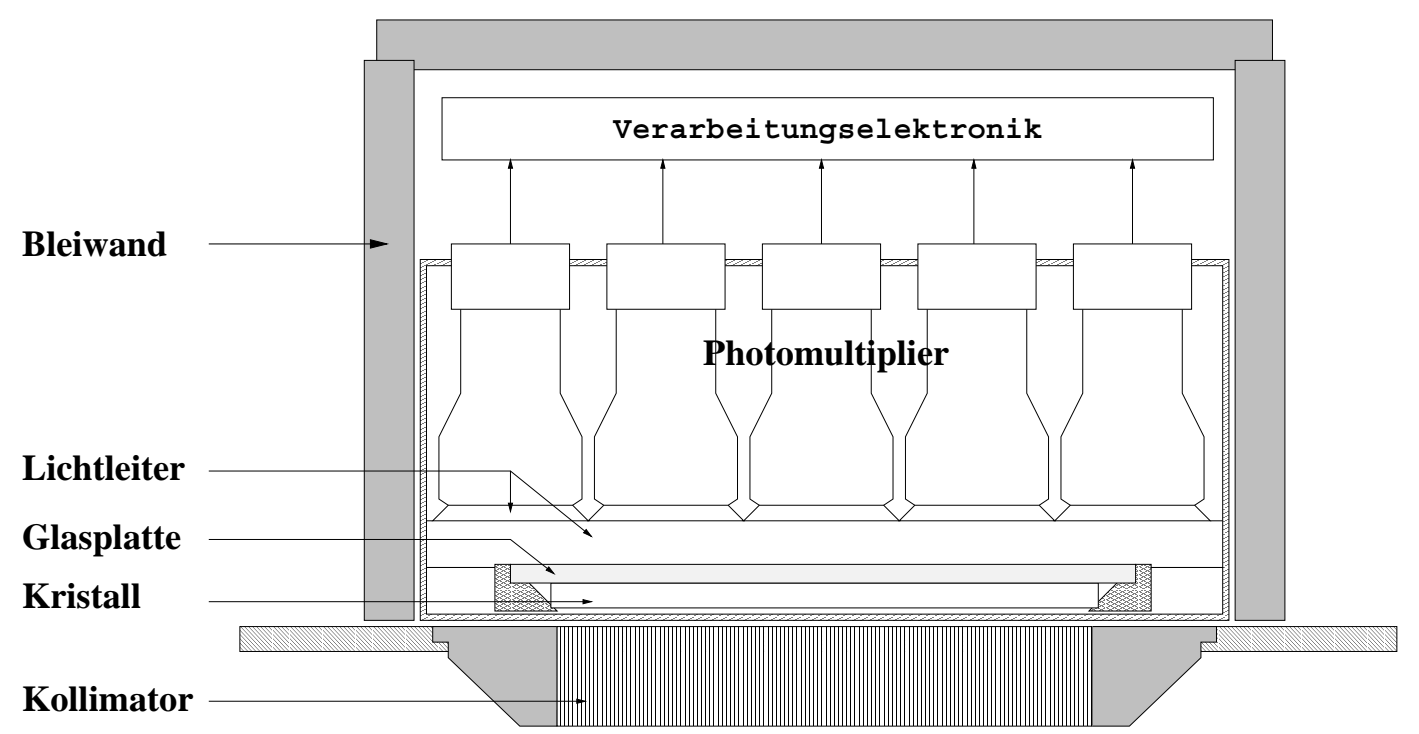

Abbildung 2.1: Schematischer Schnitt durch einen Gammakamerakopf.

von diesem Bildpunkt gesehen wird. Die szintigraphische Abbildung ist also nicht die Abbildung von Flächen wie in der Photographie, sondern stellt eine additive Projektion von Inhalten von Volumina dar ${ }^{1}$. Die Projektion der Aktivität wird gemessen, indem diejenigen Quanten, deren Richtung mit der Projektionslinie übereinstimmen, gezählt werden. Die Richtungsauswahl geschieht mit Hilfe eines Kollimators.

In Abb.2.1 ist ein schematischer Schnitt durch einen Kamerakopf mit 19 hexagonal angeordneten Photomultipliern gezeigt. Die Gammaquanten, die den Kollimator passiert haben, werden indirekt mit Hilfe eines Szintillationskristalls nachgewiesen und gezählt. Dabei wird die von dem primären Gammaquant an den Kristall abgegebene Energie in Szintillationslicht aus niederenergetischen Quanten umgewandelt, welches dann durch die Photomultiplier in Spannungssignale umgewandelt und verstärkt wird. Die Lokalisation geschieht parallel zur Zählung. Realisiert wird dies durch die Bewertung der unterschiedlichen Signale des Photomultiplierarrays, die zu einem Paar analoger Spannungen zusammengefaßt werden und deren Amplituden ein Maß für die $x$ und $y$ Koordinaten der Wechselwirkung sind. Auf die expliziten Formen der Bewertung der Signale wird in 2.2.3

1 Die Richtung der Projektion muß nicht zwangsläufig senkrecht zur Bildebene verlaufen (Parallellochkollimator), sondern es können auch Abbildungen mit Brennpunkten (z.B. Fanbeamkollimator) vorkommen. Die einfachste Form der Projektion stellt das Camera Obscura Prinzip dar, welches bei der Gammakamera mit sogenannten Pinhole-Kollimatoren umgesetzt wird. 
eingegangen.

\subsection{Beschreibung des Abbildungsvorgangs}

Es gibt eine ganze Reihe von Faktoren, die die Qualität der szintigraphischen Abbildung beeinflussen. Der Prozeß von der Emission eines Gammaquants bis zur Verarbeitung seiner erfolgreichen Detektion kann in drei Ebenen eingeteilt werden, die sich aus der Physik der betrachteten Prozesse zwanglos ergeben [31], siehe Abb. 2.2. In dieses Schema lassen sich auch die qualitätsbeeinflussenden Faktoren sinnvoll einordnen.

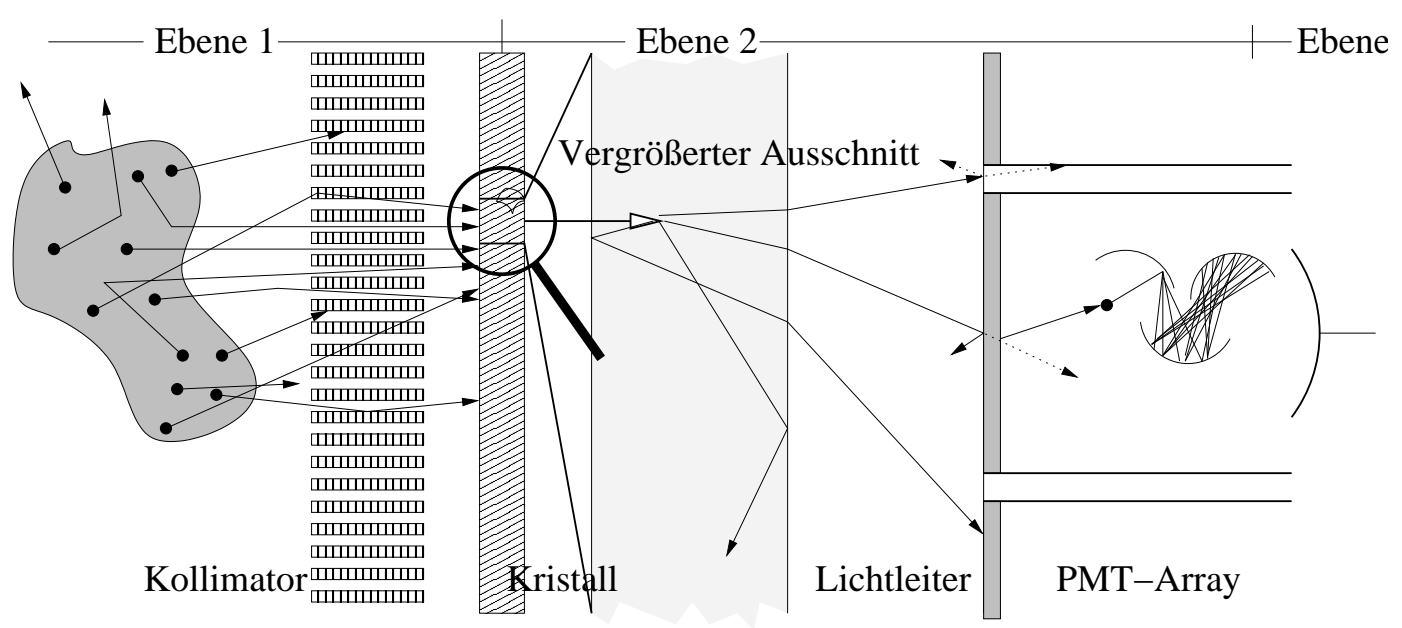

Abbildung 2.2: Die drei Ebenen der Szintigraphischen Abbildung.

1. Emission - Kristall. Der erste Schritt beinhaltet die Phänome von der Emission des primären $\gamma$-Quants bis zu dessen (möglicher) Wechselwirkung mit dem Detektionskristall. Grundlegend zur Beschreibung dieser Phänomene ist die Statistik des radioaktiven Zerfalls, also die Poissonverteilung, und die Physik der harten Röntgenstrahlung. Folgende Faktoren wirken sich qualitätsbeeinflussend aus:

- Die stochastische Natur des radioaktiven Zerfalls führt dazu, daß die Zählung der $\gamma$-Quanten nur eine Stichprobe darstellt. Somit ist dem Bild unvermeidlich ein mehr oder weniger starkes Rauschen aufgeprägt. Dieser Punkt ist insbesondere deshalb so bedeutsam, weil bei der Aufnahme von Patienten die 
applizierbare Aktivitätsmenge aus Gründen der Strahlungsbelastung sehr eingeschränkt ist und zusätzlich die Kollimation nur einen geringen Anteil der insgesamt emittierten $\gamma$-Quanten zur Registrierung zuläßt. Man muß im allgemeinen mit einem sehr geringen Signal-Rausch Abstand rechnen.

- Die Comptonstreuung führt einerseits zu falscher Ortsinformation und andererseits hat sie auch Einfluß auf die detektierte Impulshöhe, da sie einen inelastischen Prozeß darstellt. Die Comptonstreuung findet überwiegend im Patienten selbst, im Kollimator und im Szintillationskristall statt. Zusammen mit der ebenfalls vorkommenden Absorption durch Photoeffekt subsumiert man diesen schwächenden Effekt unter dem Begriff der szintigraphischen Schwächung.

- Reale Kollimatoren zeigen immer eine imperfekte Richtungsauswahl, weil die Bohrungen für eine brauchbare Ausbeute einen gewissen Durchmesser aufweisen müssen und demnach einen definierten Öffnungswinkel besitzen. Daneben sind die zur Verfügung stehenden Materialien nicht zu 100 Prozent undurchlässig für $\gamma$-Quanten (Septenpenetration). Die Durchlässigkeit steigt mit der Energie des primären $\gamma$-Quants.

2. Kristall - Photomultiplier. Im zweiten Schritt ist die Physik der Szintillation, der Lichtausbreitung innerhalb des Kristalls und der Funktionsweise der Photomultiplier von entscheidender Bedeutung.

Die Entstehung des Szintillationslichtes ist ebenfalls ein stochastischer Prozeß, d.h. die Anzahl der erzeugten Lichtquanten ist zufällig. Dies hat vor allem Einfluß auf die detektierte Impulshöhe.

Auch die Tiefe des Ortes der Szintillation innerhalb des Kristalls ist zufällig und hängt von der Energie des wechselwirkenden Gammaquants ab. Aufgrund der vielfachen Reflektionen und Spiegelungen führt dies unter Einfluß der Geometrie des Detektorsystems zu unterschiedlichen Ortsinformationen.

3. Signalverarbeitung. Neben den rein physikalischen Aspekten beeinflußt auch die Art der Verarbeitung der von den Photomultipliern zur Verfügung gestellten Signale die Qualität der Abbildung. Hier stehen vielfältige Formen der Pulsarithmetik zur Diskussion.

Da die Szintillation ein zeitlich ausgedehnter Prozeß ist, muß eine Signalverarbeitung auch entscheiden, was zu einem einzelnen Signal gehört und was nicht. Um 
richtige Ortsinformationen zu erzeugen, muß sichergestellt werden, daß das Signal nur zu einem einzelnen Ereignis gehört. Dies hat weitreichende Konsequenzen auf die Statistik: zeitlich überlappende Ereignisse müssen verworfen werden. Es handelt sich somit nicht mehr um eine Poissonstatistik.

In den nun folgenden drei Abschnitten soll auf die Einzelheiten der beschriebenen drei Ebenen eingegangen werden.

\subsubsection{Teil 1: Emission - Kristall}

Der erste Schritt beginnt bei der Emission der $\gamma$-Quanten und führt über die Betrachtung des Kollimators bis zum Erreichen des Detektionskristalls.

Der radioaktive Zerfall unter Emission eines $\gamma$-Quants ist ein stochastischer Prozeß der auch Punktprozeß genannt wird [15]. Der Begriff "Punkt" bezieht sich auf die Zeitpunkte $\left(t_{1}, \ldots, t_{n}, \ldots\right)$ des Zerfalls. Beschrieben wird der radioaktive Zerfall durch den Poissonprozeß, der wiederum durch eine einzelne Konstante $\lambda$ (hier: die mittlere Zerfallsrate $[1 / \mathrm{sec}]$ ) charakterisiert ist. Die Wahrscheinlichkeit $p_{n}(T)$, daß in einem Zeitintervall $T$ genau $n$ Zerfälle stattfinden, ist durch

$$
p_{n}(T)=\frac{(\lambda T)^{n}}{n !} e^{-\lambda T}
$$

gegeben. Im Falle der Szintigraphie bleibt $\lambda$ zwar weiterhin im zeitlichen Sinn konstant, jedoch bezieht es sich auf ein betrachtetes Volumen $V$, in dem die Zerfälle stattfinden. Die verschiedenen $\lambda\left(V_{i}\right)$ sind es, welche bildlich dargestellt werden sollen. Somit ist eines der wesentlichen Probleme der Szintigraphie angesprochen: Der stochastische Charakter des radioaktiven Zerfalls prägt dem Bild ein unvermeidliches Rauschen auf. Da die Varianz des Poissonprozesses

$$
\operatorname{Var}=\sqrt{(\lambda T)}
$$

ist, und typische Werte von $\lambda T$ für ein Szintigramm im Bereich 2 - 20 liegen, sind die stochastischen Schwankungen erheblich. Eine weitere unerwünschte Rauschkomponente kommt durch die allgegenwärtige Hintergrundstrahlung hinzu.

Um dennoch möglichst aussagekräftige Bilder zu erhalten, sucht man nach einem Verfahren, aus der gemessenen zufälligen Anzahl von Quanten eine möglichst zuverlässige und genaue Schätzung des Parameters $\lambda$ zu erhalten.

Auf dem Wege zur Gammakamera unterliegen die Gammaquanten der Wechselwirkung mit Materie. Wichtig für die Szintigraphie sind drei Prozesse: Comptonstreuung, Ray- 
leighstreuung und Photoeffekt. Alle drei Wechselwirkungen sind ebenso wie die Emission selbst stochastischer Natur.

1. Der Comptoneffekt: Dieser ist aufgrund seiner hohen Wahrscheinlichkeit von größter Bedeutung, weil er zu Bildverfälschung führt. Es handelt sich dabei um die inelastische Streuung von hochenergetischen Photonen an Elektronen. Der Comptoneffekt führt zu einer Richtungsänderung des primären $\gamma$-Quants und damit zu einer falschen Ortsinformation, weil der Ort des Zerfalls nicht in dem zu diesem Bildpunkt gehörenden Volumen liegt. Zudem kann es passieren, daß dieses Quant ohne Streuung ebenfalls detektiert und somit einem anderen Bildpunkt hätte zugeordnet werden können und nun dort fehlt. Der Effekt der Comptonstreuung ist also nicht nur eine falsch positive Zählung an dem einen Bildpunkt, sondern auch eine falsch negative an einem anderen.

Der Anteil der Quanten, die durch die Comptonstreuung fälschlicherweise einem Bildpunkt zugerechnet werden oder fehlen, kann erheblich sein und das Bild völlig unbrauchbar machen. Ein comptongestreutes $\gamma$-Quant verliert bei der Streuung Energie, und daher ist die Bestimmung der Impulshöhe die einzige Möglichkeit, gestreute Quanten zu erkennen. Um die Möglichkeit der Aussortierung dieser Quanten zu bieten, muß eine Gammakamera neben der Eigenschaft, ein ortender Zähler zu sein, auch noch spektroskopische Fähigkeiten zeigen.

2. Die Rayleighstreuung ist ein elastischer Prozeß, d.h. die Energie des Photons bleibt bei dieser Wechselwirkung erhalten. Allerdings bekommt das Photon eine andere Richtung. Die Wirkung der Rayleighstreuung wird in der Nuklearmedizin i.A. vernachlässigt, weil ihr Anteil im Vergleich zu den anderen Prozessen gering ist und die auftretenden Streuwinkel klein sind.

3. Der Photoeffekt führt zu Absorption von Photonen und damit zu Kontrastverfälschung. Der Photoeffekt äußert sich also durch das Fehlen von $\gamma$-Quanten. Zusammen mit dem oben beschriebenen Comptoneffekt bildet er die sogenannte szintigraphische Schwächung.

Die $\gamma$-Strahlung im Energiebereich zwischen $50 \mathrm{keV}$ und $500 \mathrm{keV}$ ist keinen optischen Manipulationen zugänglich. Um zu einer Abbildung zu gelangen, also um einem detektierten Quant ein Volumen $V$ zuordnen zu können, in dem der Emissionsort liegt, ist man auf 
Strahlzuordnung durch einen Kollimator angewiesen. Ein großer Nachteil eines Kollimators ist, daß die Anzahl von Quanten, die noch bildwirksam werden können, sehr gering ist. Dadurch wird das Rauschen verstärkt, und der relative Fehler, also das Verhältnis von Varianz zu Erwartungswert wird größer. Die Art der Statistik bleibt unberührt.

Neben der eklatanten Verringerung der Photonendichte zeigen Kollimatoren noch weitere, die Bildqualität entscheidend beeinflussende Eigenschaften. Dies ist z.B. die Richtungsauswahl, die nur in bestimmten Fehlergrenzen getroffen werden kann, um die Ausbeute nicht gegen Null gehen zu lassen. Da das Material eines Kollimators aus Kernen mit hoher Kernladungszahl und Dichte bestehen muß (meist Blei, seltener Wolfram), finden in ihm zusätzlich die oben beschriebenen Streuungen statt. Auch die Herstellung eines Kollimators ist nicht perfekt und kann zu lokalen Unterschieden in der Richtungsauswahl führen.

\subsubsection{Teil 2: Kristall - Photomultiplier}

Diejenigen Gammaquanten, die den Kollimator auf eine im vorhergehenden Abschnitt 2.2.1 beschriebene Weise erreicht und durchquert haben, können - müssen aber nicht - mit dem Szintillationskristall in Wechselwirkung treten. An dieser Stelle sollen die Einzelheiten derjenigen Prozesse erläutert werden, die sich vor, während und nach der Szintillation ergeben. Dabei lassen sich fünf aufeinander folgende Ebenen unterscheiden [7]:

1. Die Absorption der einfallenden Strahlung durch den Szintillator.

2. Der Szintillationsprozeß selbst, der die auf den Szintillator übertragende Energie in Lumineszenz-Photonen umwandelt.

3. Der Weg der emittierten Photonen zur Photokathode der Photomultiplier.

4. Die Absorption der Photonen an der Kathode, die Emission der Photoelektronen und deren Sammlung an der ersten Dynode.

5. Die Elektronenvervielfachung.

In dieser Reihenfolge wird nun auf die Einzelheiten der oben genannte Punkte im Detail eingegangen. 


\section{Absorption}

Der Szintillation voraus geht die Wechselwirkung des primären $\gamma$-Quants mit der Materie des Szintillationskristalls. Die $\gamma$-Strahlung wird dabei absorbiert. Quantitativ wird die Absorption durch das Lambert-Beer-Gesetz beschrieben,

$$
I=I_{0} e^{-\mu x},
$$

wobei $\mu\left[\mathrm{cm}^{-1}\right]$ der lineare Absorptionskoeffizient ist. Angeben wird oft der Massenabsoptionskoeffizient $\frac{\mu}{\rho}\left[\mathrm{g} \mathrm{cm}^{-2}\right]$, wobei $\rho$ die Dichte des Materials ist. Die Größe des Massenabsorptionskoeffizienten wird bestimmt durch die Parameter

1. Energie des primären $\gamma$-Quants und der

2. Art des Materials des Szintillationskristalls.

Die Energie des primären $\gamma$-Quants bestimmt nicht nur den numerischen Wert des linearen Absorptionskoeffizienten, sondern auch das Verhältnis der Wahrscheinlichkeiten der möglichen Prozesse wie Photo- und Comptoneffekt. Tabellen für unterschiedliche Materialien finden sich z.B. in [13].

Der für die Detektion gewünschte Prozeß ist der Photoeffekt, weil die räumliche Ausdehnung des Bereichs, in dem der Effekt innerhalb des Kristalls abläuft, klein ist und die gesamte Energie an den Kristall abgegeben wird. Die Wahrscheinlichkeit für den Photoeffekt ist eine Funktion der Kernladungszahl und der Dichte des verwendeten Materials. Für eine hohe Kernladungszahl in den gebräuchlichen Szintillationskristallen in Gammakameras aus NaI sorgt das Jod (Dichte $3.67 \mathrm{~g} / \mathrm{cm}^{3}, Z_{\text {eff }}=45$ ).

\section{Szintillationsprozeß}

Die Szintillation selbst ist kein unmittelbar auf die Energieübertragung folgender Prozeß. Die absorbierte Energie wird vielmehr von der Kristallstruktur aufgenommen. Dabei können die Elektronen entweder in das Leitungsband gehoben werden oder sog. Excitonen bilden. Excitonen sind gebundene Zustände von Elektron-Lochpaaren. Excitonen werden gebildet, wenn die Energie nicht vollständig zur Anhebung des Elektrons in das Leitungsband ausreicht oder durch Rekombination von ungebundenen Elektron-Lochpaaren. Die Energie der Elektronen im Leitungsband oder der Excitonen kann an sog. Szintillationszentren oder -keimen in Licht umgewandelt werden. Konkurierend dazu existieren 
andere, ohne Lichtabstrahlung verbundene Prozesse die unter dem Begriff Quenching zusammengefaßt sind. Will man $\gamma$-Spekroskopie mit einer Gammakamera betreiben, so ist zu berücksichtigen, daß die Summe der Impulshöhen nicht unmittelbar die Energie der $\gamma$-Quanten repräsentieren, sondern die der Elektronen und Excitonen, die aus den Wechselwirkungsprozessen der $\gamma$-Quanten stammen. So ist die Proportionalität zur Energie des primären $\gamma$-Quants nur annähernd gegeben[12]. Aus diesem Grunde ist die Verwendung der Impulshöhen als Energieäquivalent problematisch.

Eine Folge des mittelbaren Charakters der Szintillation ist die damit verbundene, vergleichsweise große zeitliche Spanne, die dieser Prozeß in Anspruch nimmt. Eine andere Folge ist, daß der Ort der Energieübertragung und der Ort der Szintillation(en) nicht derselbe ist, und es stellt sich die Frage nach der räumlichen Ausdehnung. Glücklicherweise ist im Rahmen der Szintigraphie der Vorgang trotz der erwähnten Zusammenhänge als quasi-punktuell zu betrachten: Beim Photoeffekt liegen die an der Lichtaussendung beteiligten Szintillationskeime in einem Raumgebiet mit einem Durchmesser von weniger als einem Millimeter, weil die mittlere Reichweite von Elektronen im betrachteten Energiebereich sehr klein $\left(<10^{-2} \mathrm{~g} / \mathrm{cm}^{2}\right)$ ist.

Szintillationskeime stellen im reinen NaI-Kristall die Kristallfehlstellen dar, es sind also schon am undotierten Kristall Szintillationserscheinungen zu beobachten. Die Effektivität der Lichtumwandlung läßt sich jedoch deutlich durch Einbau von Fremdatomen in den Kristallverband steigern; diese übernehmen die Rolle der Fehlstellen und erhöhen dabei nicht nur die Wahrscheinlichkeit eines optischen Übergangs, sondern auch die Anzahl der erzeugten Lumineszens-Phontonen. Hier hat sich das $\mathrm{Tl}$ als besonders effektiv gezeigt, weshalb nahezu alle praktisch verwendeten Detektionskristalle aus NaI mit Thallium „aktiviert" sind. Das Thallium zeigt zusätzlich das Vermögen, die mittlere Abklingzeit der Szintillation von etwa $3.5 \cdot 10^{-7}$ Sek. auf $2.5 \cdot 10^{-7}$ Sek. herabzusetzen [10]. Ebenfalls wird das Spektrum der Szintiallation günstig beeinflußt, indem die ultravioletten Anteile, die beim reinen $\mathrm{NaI}$ das Maximum ausmachen, zugunsten langwelliger Anteile (Maximum bei etwa $430 \mathrm{~nm}$ ) fast vollständig unterbunden werden [35]. Diese Beeinflussung ist deshalb so günstig, weil die spektrale Empfindlichkeit der Photomultiplier im langwelligeren Bereich deutlich höher ist [7].

Im Mittel wird bei $\mathrm{NaI}(\mathrm{Tl})$ ein Lichtquant pro $50 \mathrm{eV}$ des primären $\gamma$-Quants erzeugt [7]. Dies bedeutet bei vollständiger Abgabe der Energie in etwa 2800 bzw. 10000 Quanten für ein ${ }^{99 m} \mathrm{Tc}$ bzw. $511 \mathrm{keV}$ (Positronen-Zerfall, z.B. ${ }^{18} \mathrm{~F}$ ) $\gamma$-Quant.

Die beiden entscheidenden Punkte der Physik des Szintillationsprozesses sind der Zu- 
fallscharakter der Anzahl der erzeugten Lumineszenz-Photonen und die zeitliche Dauer der Szintillation. Ersteres hat Einfluß auf das Auflösungsvermögen der Impulshöhe, wohingegen Zweiteres das zeitliche Auflösungsvermögen der Gammakamera beeinflußt und nicht nur zu falschen Zählungen, sondern auch zu falschen Orts- und Impulshöheninformationen führt. Dies hat weitreichende Konsequenzen auf die Zählratenstatistik, weil die Filterung zeitlich zu dicht beieinander liegender Ereignisse sämtliche daran beteiligte Impulse verwerfen muß. Dieser Zusammenhang wird in Kapitel 4.3 noch ausgiebig erörter.

\section{Geometrie und Elektronenvervielfachung}

Die Szintillationsquanten unterliegen ebenfalls verschiedenen Einflüssen. Vom Szintillationszentrum ausgehend, können sie durch Totalreflektion an den Übergängen Kristall/Lichtleiter und Lichtleiter/Photokathode und durch Spiegelung an der kollimatorseitigen Versiegelung des Detektionskristalls (z.B. Aluminium) mehrfach das Systems Kris-

Abbildung 2.3:

Mögliche beteiligte Prozesse. Szintillation(1), isotrope Lichtausbreitung(2), Lichtweg durch Kristall(3) und Lichtleiter(4), Absorption auf der Photokathode(6), Reflektionen(5,3), Absorptionen (4,7) und Verlust (8). Entnommen aus [26].

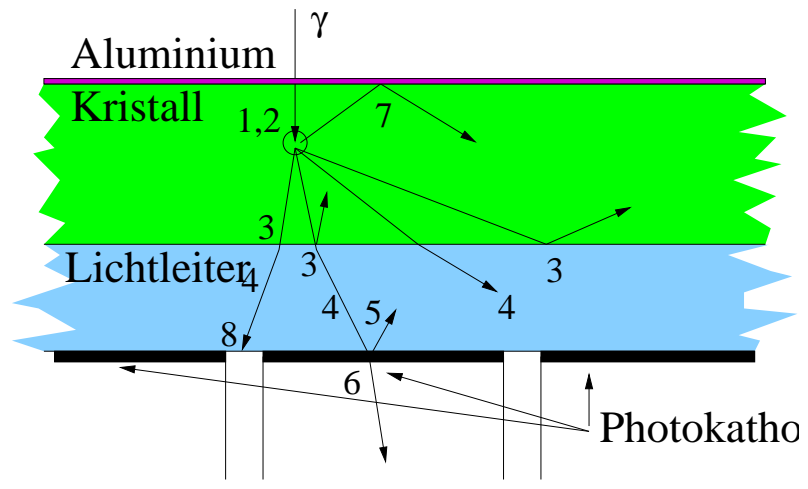

tall/Lichtleiter durchlaufen, bevor sie an der Photokathode absobiert werden. Diese und weitere Möglichkeiten sind in Abbildung 2.3 [26] aufgeführt. Es ist klar, daß durch diese Prozesse die Geometrie des System Kristall/Lichtleiter, also z.B. die Dicke von Kristall und Lichtleiter Einfluß auf die Gestalt der Signale hat.

Als Beispiel für die Gestalt von Signalen einzelner Photomulitplier als Konsequenz der geometrischen Verhältnisse ist die Abbildung 2.4 aufgetragen. Mit Hilfe der Feinnadelstrahlquelle (Eigenschaften siehe Abschnitt 3.1.1) ist es möglich, das Signal einzelner Photomultiplier als Funktion des Abstandes des Szintillationszentrums von der Achse des Photomultipliers zu vermessen. Dazu wird die Feinnadelstrahlquelle entlang der roten Linie, die im rechten Teilbild der Abbildung 2.4 eingezeichnet ist, in Abständen von $5.3 \mathrm{~mm}$ verfahren und für jede Position jeweils zehnmal das Signal mit Hilfe eines Oszilloskops 


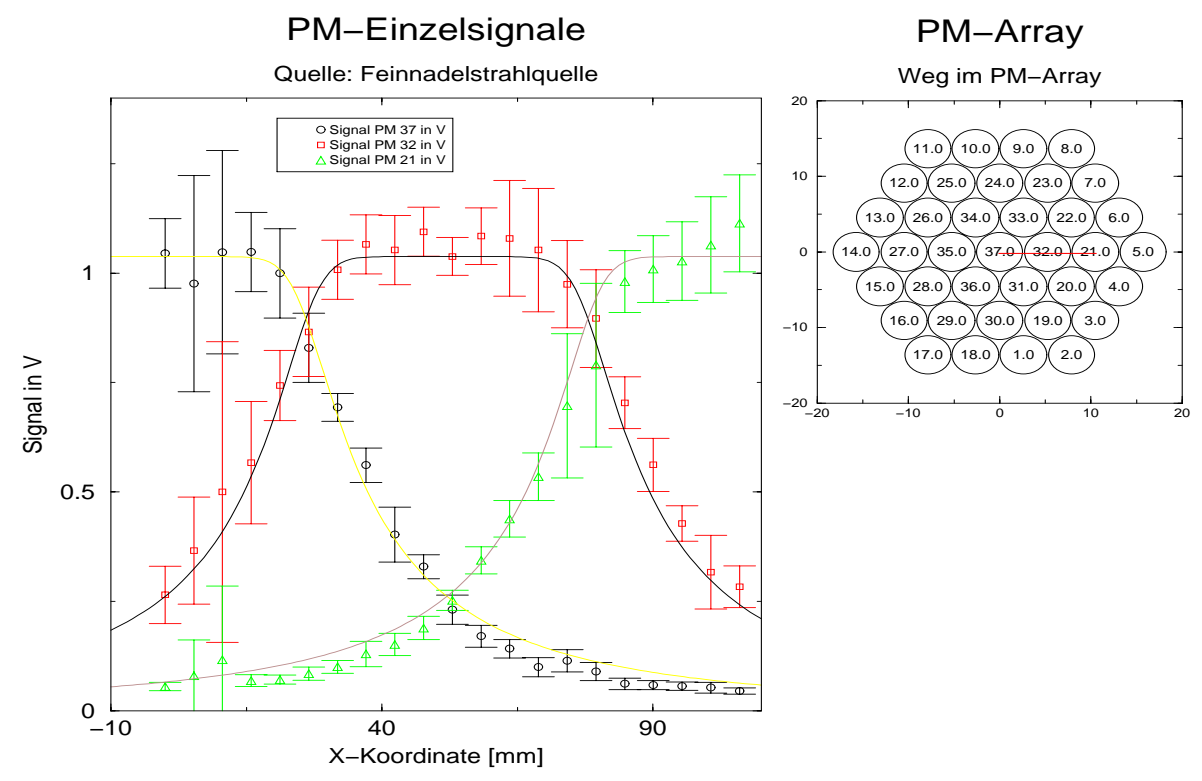

Abbildung 2.4: Signale der drei Photomultiplier 37,32 und 21 als Antwort auf unterschiedliche Positionen der Feinnadelstrahlquelle. Diese ist auf der roten Achse des rechten Teilbildes in Abständen von $5.3 \mathrm{~mm}$ verfahren worden. Die durchgezogenen Linien im linken Teilbild entsprechen Fits mit der Funktion (2.4).

abgenommen. Im linken Teilbild der Abbildung 2.4 sind die Mittelwerte und Standardabweichung der Antworten der drei Photomultiplier 37,32 und 21 einer Gammakamera vom Typ Picker CX-250 dargestellt.

Zusätzlich zu den Meßwerten ist eine Funktion aufgetragen, die einen Fit nach kleinsten Fehlerquadraten an die jeweilige Antwort der Photomultiplier darstellt. Die Gestalt dieser Funktion ist

$$
f(x)=\frac{a}{\left(1+\left(\frac{\left|x-x_{0, i}\right|}{r}\right)^{b}\right)^{c}} .
$$

Die freien Parameter des Fits stellen $a, b$ und $c$ dar. Der Parameter $r$ stellt den Radius der Photokathode und $x_{0, i}$ die Koordinate der Achse eines Photomultipliers $i$ dar. Die Kenntnis der Funktion (2.4) und der in ihr enthaltenen Parameter kann zur Simulation des Abbildungsvorgangs eingesetzt werden.

Besonders bemerkenswert an der Auftragung 2.4 ist die relativ kleine Schwankung der Signale bei Variation des Abstandes der Feinnadelstrahlquelle in der Nähe der Achse des Photomultipliers. Diese ist nicht auf Besonderheiten in der Geometrie des KristallLichtleiter Systems zurückzuführen, sondern hat seine Ursache in der Existenz einer sog. Maske zwischen Lichtleiter und Kristall. Die Maske besteht aus geschwärzten Ab- 
schnitten auf der Oberfläche des Lichtleiters, die den Lichtfluß beeinflußt. Eine solche Maske stellt die erste Form von permanenter Korrektion des Abbildungverhaltens dar, da mit ihrer Hilfe ein Besserung der Nichtlinearitäten zu erreichen ist. Ein großer Nachteil dieses Vorgehens besteht in dem Verzicht auf Teile des Szintillationslichtes mit der Folge eines geringeren Signal-Rausch Abstandes.

\subsubsection{Teil 3: Signalverarbeitung}

Nach der Verstärkung durch die Photomultiplier stehen entsprechend der Anzahl der Photomultiplier $N$ verschiedene Spannungssignale zur Bestimmung des Detektionsortes und der Impulshöhe zur Verfügung. Durch arithmetische Verknüpfung der in bestimmter Weise gewichteten Signale enstehen die Orts- und Impulshöhenkoordinaten. Jedem Photomultiplier mit der Nummer $i$ werden Gewichte $q_{x i}$ bzw. $q_{y i}$ zugewiesen, die proportional zu der $x$ - bzw. $y$-Koordinate der Mittelpunkte der Photokathode des jeweiligen Photomultipliers sind. Der Ursprung dieses Koordinatensystems liegt meist zentral auf dem Kristall, fällt bei runden Gammakamaraköpfen also mit dem Mittelpunkt der Photokathode des zentralen Photomultipliers zusammen.

Abbildung 2.5:

Positionen für eine Probeaktivität zur Bestimmung der $g_{i^{-}}$ Faktoren zum Abgleich der Impulshöhenantwort der Gammakamera. Der Kreis markiert den zugänglichen Bereich.

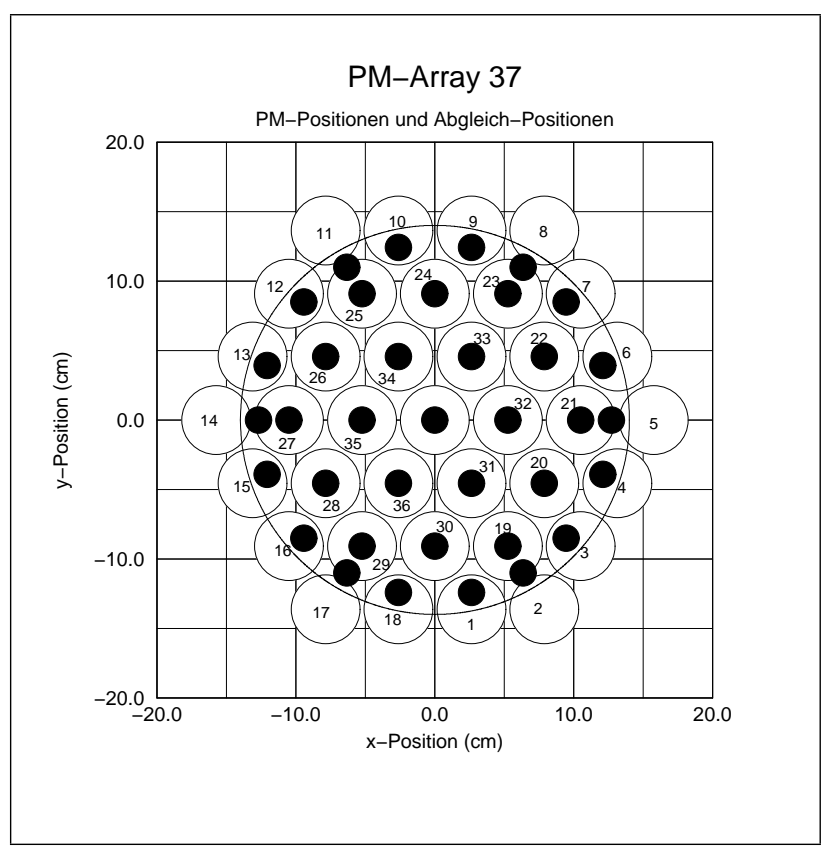

Aus den im vorhergehenden Abschnitt 2.2.2 beschriebenen Gründen würde man bei der Bestimmung der Impulshöhe durch Addition aller Einzelsignale eine örtlich stark va- 
riierende Antwort erhalten. Deshalb werden die Einzelsignale bei der Berechnung der Impulshöhe ebenfalls mit einem Faktor $g_{i}$ bewertet. Diese sind so gewählt, daß eine möglichst homogene Impulsantwort entsteht. Praktisch werden diese Faktoren bestimmt, indem nacheinander jeweils zentral vor einem Photomultiplier eine Probeaktivität positioniert wird und die Wichtungsfaktoren solange verändert werden, bis das Summensignal für alle Positionen gleich ist. Dies Verfahren entspricht dem Lösen eines linearen Gleichungssystems $A_{i j} \cdot g^{i}=$ konst, wobei $g_{i}$ den Wichtungsfaktor des $i$-ten Photomultipliers darstellt und $A_{i j}$ eine Matrix ist, deren Spalten $i$ aus den Signalen des Photomultipliers $i$ in Abhängigkeit von den $j$ Positionen der Probeaktivität.

Da die Signale von Photomultipliern, die relativ weit entfernt zum Szintillationszentrum stehen, so klein sind, daß neben dem Rauschen so gut wie keine Information im Signal enthalten ist, wird oft auf diese Signale völlig verzichtet. Implementiert wird dies durch eine Schwelle, die ein Signal mindestens überschreiten muß, damit es in die Berechnungen eingeht. Man erreicht so einen etwas höheren Signal-Rausch Abstand, vor allem zum Rand des Detektionskristalls hin.

Tatsächlich stehen mehrere alternative Impulsarithmetiken zur Auswahl um an die Orts- und Impulshöheninformation zu gelangen. Sie können in fünf verschiedene Klassen eingeteilt werden [31, 6], wobei hier eine Schwellenwertberücksichtigung nicht explizit mit angegeben wird. In den Gleichungen (2.5) stellen $X, Y$ und $Z$ die von der Kamera ausgegebenen (und an nachgeschaltete Verarbeitungseinheiten weitergereichte) Signale dar, die als Orts- bzw. Impulshöhenkoordinatenwerte interpretiert werden. Werden keine Wichtungsfaktoren $g_{i}$ bestimmt für die Impulshöhensumme (PA I und PA IV), so werden die $q_{x i}$ und $q_{y i}$ durch $p_{x i}$ und $p_{y i}$ zur besseren Unterscheidbarkeit ersetzt. Neben dem mit Hilfe der $g_{i}$ gebildeten Impulshöhensummensignals $Z$ verwenden einige Impulsarithmetiken auch noch das einfache ohne Wichtung der Signale der einzelnen Photomultiplier gebildete Impulshöhensummensignal $Z^{\prime}$, welches manchmal anstelle von $Z$ verwendet wird, um durch Division die Ortssummensignale unabhängig von der Energie des $\gamma$-Quants zu machen.

$$
\text { PA I }\left\{\begin{array} { r l } 
{ X } & { = \sum _ { i = 1 } ^ { N } p _ { x i } V _ { i } } \\
{ Y } & { = \sum _ { i = 1 } ^ { N } p _ { y i } V _ { i } } \\
{ Z } & { = \sum _ { i = 1 } ^ { N } V _ { i } , }
\end{array} \quad \text { PA II } \left\{\begin{array}{rl}
X & =\sum_{i=1}^{N} q_{x i} g_{i} V_{i} \\
Y & =\sum_{i=1}^{N} q_{y i} g_{i} V_{i} \\
Z & =\sum_{i=1}^{N} g_{i} V_{i},
\end{array}\right.\right.
$$




$$
\begin{aligned}
& \text { PA III }\left\{\begin{aligned}
X & =\sum_{i=1}^{N} q_{x i} g_{i} \frac{V_{i}}{Z} \\
Y & =\sum_{i=1}^{N} q_{y i} g_{i} \frac{V_{i}}{Z} \\
Z & =\sum_{i=1}^{N} g_{i} V_{i},
\end{aligned}\right. \\
& \text { PA IV }\left\{\begin{array} { r l } 
{ X } & { = \sum _ { i = 1 } ^ { N } p _ { x i } \frac { V _ { i } } { Z ^ { \prime } } } \\
{ Y } & { = \sum _ { i = 1 } ^ { N } p _ { y i } \frac { V _ { i } } { Z ^ { \prime } } } \\
{ Z ^ { \prime } } & { = \sum _ { i = 1 } ^ { N } V _ { i } } \\
{ Z } & { = \sum _ { i = 1 } ^ { N } g _ { z i } V _ { i } , }
\end{array} \quad \mathrm { PAV } \quad \left\{\begin{array}{rl}
X & =\sum_{i=1}^{N} q_{x i} \frac{V_{i}}{Z^{\prime}} \\
Y & =\sum_{i=1}^{N} q_{y i} \frac{V_{i}}{Z^{\prime}} \\
Z^{\prime} & =\sum_{i=1}^{N} V_{i} \\
Z & =\sum_{i=1}^{N} g_{z i} V_{i} .
\end{array}\right.\right.
\end{aligned}
$$

Die Realisation der obigen Arithmetiken erfolgt in älteren Gammakameras mit Hilfe einer analogen Elektronik und in neueren Gammakameras bereits auf digitale Art. Historisch bedingt nennt man deshalb die Arithmetiken PA I bis PA II auch Differenzschaltungen (difference-circuits) und die Arithmetiken PA III bis PA IV Verhältnisschaltungen (ratio-circuits). Die Verhältnisschaltungen zeichnen sich durch eine deutlich verringerte Verzerrung in der Ortsbestimmung aus. Darüberhinaus zeigen sie im Gegensatz zu den Differenzschaltungen keine andere Skalierung des Bildes für unterschiedliche $\gamma$-Energien. Deshalb werden heute Verhältnisschaltungen den Differenzschaltungen vorgezogen [32]. Die für diese Arbeit zur Verfügung stehende Gammakamera vom Typ Picker CX-250 realisiert die Arithmetik PA III.

\subsection{Charakterisierung der Abbildungsfehler}

Zwei Typen von Fehlern können unterschieden werden. Das begrenzte zeitliche Auflösungsvermögen kann dazu führen, daß die Szintillationen aufgrund der gleichzeitigen ${ }^{2}$ Wechselwirkung mehrerer $\gamma$-Quanten mit dem Kristall als ein einziges Ereignis detektiert werden, mit Koordinatenwerten, die einer Art Schwerpunkt der Koordinaten der einzelnen $\gamma$-Quanten entsprechen. Die Abbildung 2.6 zeigt beispielhaft zwei punktförmige Quellen, die direkt auf den Detektionskristall gebracht sind. Auf der Verbindungslinie zwischen den Schwerpunkten der beiden Verteilungen sind diejenigen Ereignisse lokalisiert, die nicht einem einzelnen Quant entsprechen. Hier kann man nicht von einem Fehler als Abweichung von einem wahren Wert sprechen, sondern schlicht von falschen Werten. Diese Ereig-

\footnotetext{
${ }^{2}$ Gleichzeitig im Sinne eines zeitlichen Abstandes, der kleiner als die Verarbeitungszeit ist.
} 
Abbildung 2.6:

Zwei punktförmige Quellen mit sehr hoher Aktivität. Die Quellen befinden sich direkt auf dem Detektionskristall. Bei sehr hohen Aktivitäten funktioniert der Verwerfungsmechanismus nicht mehr perfekt und es kommt zu dem „Geisterbild“ entlang der Verbindungslinie zwischen den beiden Quellen. Die Verschmierung zu den äußeren horizontalen Rändern ist eine Folge der Verzerrung der Abbildung an den Rändern der Gammakamera.

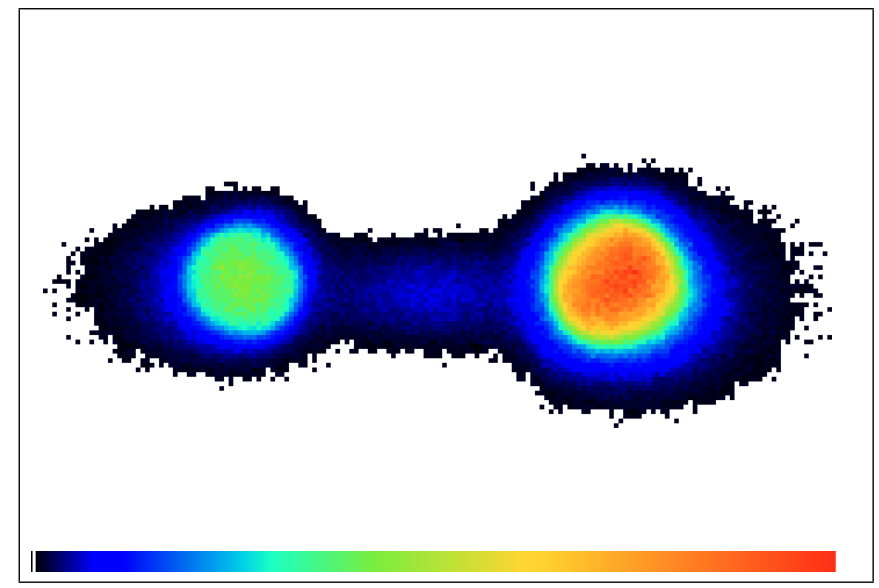

nisse müssen und werden von der Verarbeitungselekronik verworfen und können nicht korrigiert werden. Arbeitet der Verwerfungsmechanismus hinreichend genau, so ist der bildverfälschende Effekt über den gesamten Kristall hinweg der eines multiplikativen Faktors $(<1.0)$ und wird deshalb an dieser Stelle nicht weiter diskutiert, jedoch findet sich eine ausführliche Betrachtung in Kapitel 4 wo insbesondere in 4.3 der Überlagerungsmechanismus im Detail dargestellt ist.

Für den zweiten Fehlertyp sei die Situation betrachtet, in der ein einzelnes $\gamma$-Quant mit dem Detektorkristall wechselwirkt und eine den Anstoß für eine Signalverarbeitung gibt. Dabei soll es zeitlich derart isoliert sein, daß die Verarbeitung ungestört von anderen Ereignissen verlaufen kann. Auch unter diesen Bedingungen kommt es aufgrund der in den vorangegangenen Kapiteln beschriebenen Prozesse bei der Impulshöhen- und Ortszuweisung Abweichungen vom gewünschten linearen Verhalten, obwohl das $\gamma$-Quant im Sinne eines einzelnen Ereignisses richtig detektiert wurde. Die unrichtigen Impulshöhenund Ortsinformationen im Sinne einer linearen Abbildung führen zu Bildverfälschungen. Im folgenden werden diese Verfälschungen ebenfalls als Fehler bezeichnet, auch wenn es nicht die Gammakamera ist, die fehlerbehaftet ist, sondern die erst die Interpretation ihres Abbildungsverhaltens als lineare Abbildung nicht korrekt ist. Die Tatsache, daß diese Fehler reproduzierbar sind, also die Annahme, daß auf identische physikalische Ereignisse im Kristall immer wieder die gleiche Impulshöhen- und Ortszuweisung - im Sinne von Erwartungswerten - erfolgt, macht diese Fehler einer Korrektion zugänglich.

Die bildwirksame Folge der Fehler bei der Detektion sind auf der einen Seite Verzerrungen und auf der anderen Seite falsche Bildpunktintensitäten.

Falsche Bildpunktintensitäten, die auch unter dem Begriff Inhomogenität des Detek- 
torsystems bekannt sind, können sichtbar gemacht werden, in dem der Detektionskristall einer flächenhaften homogenen Strahlung ausgesetzt wird. Ein Kollimator kommt hierbei nicht zum Einsatz. Eine flächenhaft homogene Strahlung wird in der Praxis oft durch eine hinreichend weit entfernte Punktquelle angenähert realisiert. Liegt eine Inhomogenität in der Zuweisung der Bildpunktintensitäten jenseits der statistischen Schwankungen vor, so zeigt sich dies in der örtlichen Variation der nachgewiesenen Aktivität. In einem Graustufenbild stellt sich dies durch hellere oder dunklere zusammenhängende Gebiete dar.

\begin{abstract}
Abbildung 2.7:
Aufnahme einer fernen Punktquelle. Deutlich zeichnen sich die Photomultiplier ab. Die rote Kurve kennzeichnet den vom Hersteller angegebenen maximal zu verwendenden Bereich. Es ist weder ein Kollimator verwendet, noch sind irgendwelche Korrektionen angewendet worden.
\end{abstract}

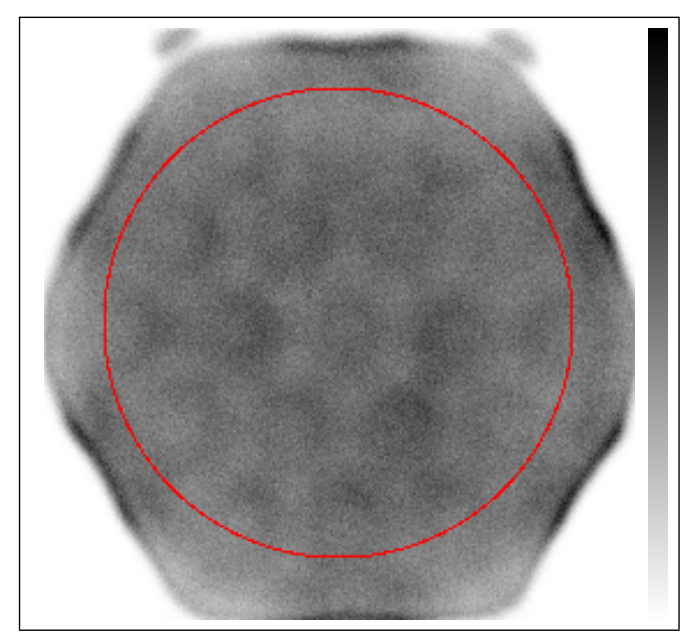

Der intuitiv naheliegendste Grund für eine Inhomogenität scheint in der unterschiedlichen Nachweiswahrscheinlichkeit an unterschiedlichen Orten des Kristalls zu liegen. Allerdings ist dieser Effekt von weitaus kleinerer Ordnung als die folgenden. Die beiden wesentlichen Gründe für das Auftreten von Inhomogenitäten sind die Nichtlinearität der Abbildung und die Variation der Impulshöhendetektion bei gleichzeitiger Verwendung eines Impulshöhenfensters.

Die Nichtlinearität ist die zur Zuweisung von Ereignissen an einen falschen Bildort, so daß einige Bildelemente (Pixel) mehr, andere weniger Ereignisse zugewiesen bekommen. Es ist möglich, dies als unzureichende Pixeldefinition in dem Sinne zu betrachten, daß das künstliche Pixelraster mit gleich großen Rasterelementen dem Abbildungsverhalten der Gammakamera nicht angepaßt ist. Das im folgenden vorgestellte Korrektionsverfahren setzt hier an und definiert auf Grundlage der Messungen mit der Feinnadelstrahlquelle ein passendes Raster, siehe 3.1.3.

Die örtlichen Unterschiede der Impulshöhendetektion führen bei Anlegen eines Im- 
pulshöhenfensters zu Inhomogenitäten, weil durch die Variation der Impulshöhenverteilung unterschiedliche Anteile verworfen werden. Eine Skizze dazu findet sich in der Abbildung 3.1 auf Seite 30. Die richtige Impulshöhendetektion ist ein für die Qualität der Szintigramme von großer Bedeutung.

Ein weiterer Effekt der Variation der Impulshöhenverteilung ist neben einer Verschiebung der Verteilung als ganzes auch eine Stauchung oder Streckung. Die Folge ist, daß ein örtlich konstantes Impulshöhenfenster zu Inhomogenitäten führen kann. Allerdings ist dieser Fehler von geringerer Ordnung als der vorher beschriebene Effekt. Soweit bekannt, berücksichtigen gängige Impulshöhenkorrekturen nur den Effekt der Verschiebung der Impulshöhenverteilung als ganzes.

Die Nichtlinearität in der Ortszuweisung ist eine direkte Folge der Nichtlinearität der Antworten der einzelnen Photomultiplier auf ein Szintillationsereignis in Abhängigkeit des lotrechten Abstandes des Szintillationszentrums von der Achse eines Photomultipliers. Darüberhinaus ist sie eine Folge der Endlichkeit des Photomultiplierarrays. Diese ist natürlich vor allem an den Rändern der Kamera spürbar und führt zu einer deutlichen Einschränkung des sinnvoll zu nutzenden Detektionsbereichs.

\subsection{Simulation}

In diesem Abschnitt soll die numerische Simulation des Abbildungsprozesses einer Gammakamera vorgestellt werden.

Ziel der Simulation ist es, Zugang zu verschiedenen Parametern des Abbildungsprozesses zu erhalten, die bei der realen Kamera gar nicht oder nur sehr schwer einzeln zu beeinflussen sind. Durch die gezielte Variation einzelner Parameter ist eine definierte Bildbeeinflussung möglich, anhand derer die Wirksamkeit und Reichweite von Korrektionsverfahren getestet werden können, ohne daß der Einsatz radioaktiven Materials notwendig ist. Daneben ist so eine tieferes Verständnis des Abbildungsprozesses erreichbar.

Es war nicht Ziel dieser Arbeit, alle drei Ebenen der Szintigraphie, wie sie in Abschnitt 2.2 beschrieben sind, in die Simulation mit einzubeziehen. Die Prozesse der Ebene 1 und z.T. 2 sind eingehend mit Hilfe der Monte-Carlo Simulation untersucht, allgemein z.B. in [3] und speziell auf die Szintigraphie mit Gammakameras bezogen in [23]. Es existieren zudem kommerzielle Computerprogramme GEANT und EGS4. Selbstverständlich können die Ergebnisse solcher Simulationen als Eingang der hier zu beschreibenden Simulation dienen. 
Es sind vor allem die Phänomene der Ebenen 2 und 3, die besondere Berücksichtigung finden sollen. Die Modellierung setzt sich entsprechend zusammen aus

- der Bestimmung der Eindringtiefe des primären $\gamma$-Quants in den Kristall nach der Exponentialverteilung mit dem linearen Absorptionskoeffizienten des Kristallmaterials als Parameter,

- der Lichtausbreitung im Kristall und im Lichtleiter,

- der Berechnung des Signals eines einzelnen Photomultipliers in Abhängigkeit von der Entfernung des Szintillationszentrums und der Energie des primären $\gamma$-Quants und

- der Pulsarithmetik der zu einem einzelnen Ereignis gehörenden Impulse zur Ortsund Impulshöhenbestimmung.

\subsubsection{Das Modell}

Den Ausgangspunkt der Simulation bildet das als näherungsweise punktförmig angenommene Zentrum der Szintillation mit der senkrechten Eindringtiefe $z_{e}$. Das Signal eines einzelnen Photomultipliers wird proportional zu dem Integral der Intensität des Lichts gesetzt, welches auf die Photokathode des Photomultipliers fällt.

Die Lichtausbreitung der Szintillation im Kristall wird als isotrop angenommen. Die Unterkante des Kristalls wird als Spiegel, die seitlichen Begrenzungen wie die Photokathoden werden als idealer Absorber behandelt. Der Übergang von Kristall zu Lichtleiter wird durch die Fresnelschen Gleichungen beschrieben. In der Abb. 2.8 ist dieser Zusammenhang schematisch dargestellt. Zusätzlich zum direkten Strahlengang ist die erste Spiegelung an der Aluminiumfolie eingezeichnet, die den hygroskopischen Kristall umschließt.

Neben der einfachen Spiegelung an der Aluminiumfolie gibt es natürlich noch weitere Mehrfachreflektionen, die unter Umständen einen nicht zu vernachlässigenden Anteil an der Intensität haben können, die auf die Photokathoden der Photomultiplier treffen. Diese Anteile werden mit Hilfe der durch Spiegelung entstehenden virtuellen Szintillationszentren berücksichtigt.

Die Berechnung der Intensität $I$ auf einer Photokathode geschieht mittels Integration über die Fläche der Photokathode entlang der Kreislinien konstanter Intensität. Die Flächenelemente $d F$ ergeben sich als der Schnitt zwischen der Kreisfläche der Photokathode einerseits und der konzentrisch um das Lot des Szintillationszentrums auf die 
Abbildung 2.8:

Ausleuchtung der Photokathode. Dargestellt ist der direkte Anteil des vom Szintillationszentrum $S Z$ ausgehenden Lichts sowie derjenige Anteil, der durch einfache Spiegelung an der Aluminiumfolie entsteht. Dieser besitzt ein virtuelles Szintillationszentrum außerhalb des Kristalls $S Z_{v}$.

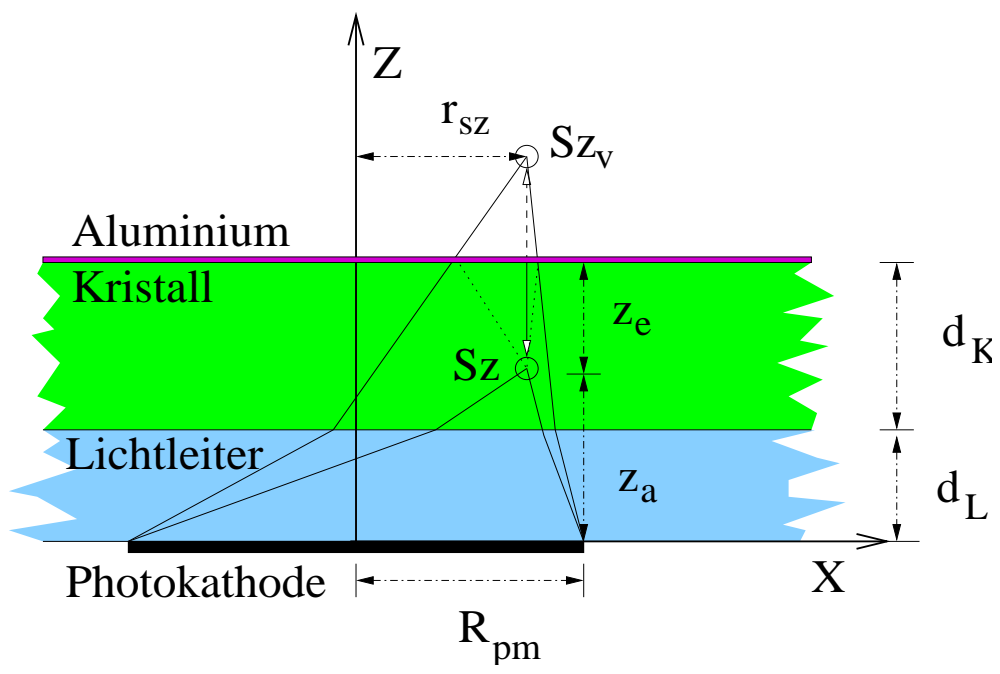

Ebene der Photokathoden verlaufenden Kreisringen konstanter Intensität andererseits. Zur Verdeutlichung des Sachverhalts ist Abb. 2.9 aufgetragen.

Abbildung 2.9:

Zur Bestimmung der Intensität. Auf der Kreissegment mit der Fläche $d F$ ist die Intensität konstant, daher kann leicht über den Winkel $2 \gamma$ integriert werden. Es muß nur die Abhängigkeit der Intensität $I$ vom Radius $r$ und der Winkel $\gamma$ berechnet werden.

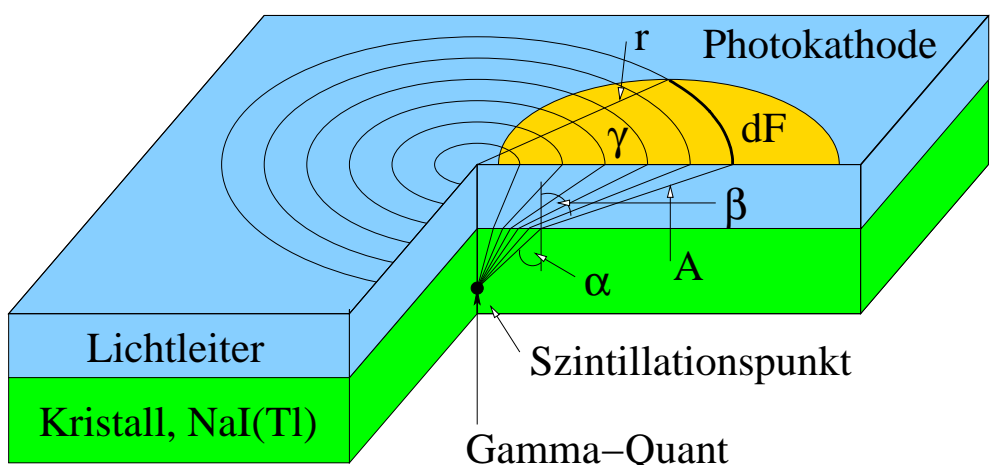

Der Abstand $r$ des Lots des Szintillationszentrums auf die Ebene, in der die Photokathoden liegen, bestimmt die Brechungswinkel $\alpha$ und $\beta$ und damit die Länge des Lichtweges $L W$ vom Szintillationszentrum zum Flächenelement $d F$. Die Bestimmung dieser Größen muß allerdings numerisch mit Hilfe des Brechungsgesetzes aus der Eindringtiefe $z_{e}$ und des Abstandes $r$ erfolgen. Die konkrete Berechnung sowie die Berücksichtigung mehrfach reflektierter Anteile finden sich in Anhang A.1 bzw. A.2. Das Gesamtsignal eines Photomultipliers in Abhängigkeit vom horizontalen Abstand des Szintillationszentrum (geo- 
Abbildung 2.10:

Aus der Berechnung der Intensität des Szintillationslichts gewonnene Funktionen des Signals eines Photomultipliers in Abhängigkeit des horizontalen Abstands des Szintillationszentrums zur Achse des Photomultipliers. Dargestellt sind Funktionen, die zu unterschiedlichen vertikalen Abständen des Szintillationszentrums zur Photokathode gehören.
Geometrische Effizienz

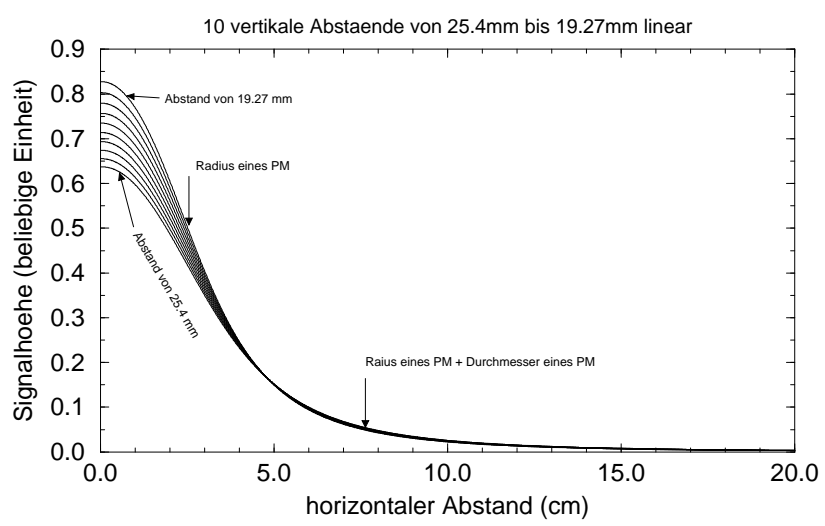

metrische Effizienz) ist in der Abbildung 2.10 aufgetragen. Ohne die Verspiegelung wäre die Abhängigkeit von der Eindringtiefe noch größer. Der gewonnene Verlauf ist in guter Übereinstimmung mit Berechnungen, die auf andere Weise gewonnen wurden [31, 25, 27]

\subsubsection{Szintigramme aus der Simulation}

Gibt man den Ort des Szintillationszentrums vor, so kann mit Kenntnis der Signale der einzelnen Photomultiplier und der Wahl einer Impulsarithmetik aus (2.5) sowohl die Ortung als auch die Impulshöhenzuweisung durch eine Gammakamera simuliert werden, in der die gleiche Impulsarithmetik zum Einsatz kommt. Wiederholt man dies für eine Reihe von $\gamma$-Quanten, mit entprechend gewählten Szintitillationszentren, so kann das dazugehörige Szintigramm erstellt werden.

Um den stochastischen Prozessen Rechnung zu tragen, ist die Vorgehensweise ist die, daß man aus den vorgegebenen möglichen Auftrefforten zufällig einen Ort mit einer vorgegebenen Wahrscheinlichkeit auswählt und diesen als Auftreffort eines Quants betrachtet. Danach ist die Eindringtiefe ebenfalls zufällig, dem Lambert-Beerschen Gesetzt entsprechend, zu bestimmen. Jetzt liegen alle Informationen vor, um die Signale der einzelnen Photomultiplier als Antwort auf die Szintillation an dem entsprechenden Ort zu berechnen und mit einem zufälligen Fehler aus einer vorzugebenden Verteilung zu versehen. In den hier durchgeführten Simulationen ist das Rauschen der Photomultipliersignale stets über eine Gaußverteilung simuliert worden. Die jeweiligen Auswirkungen der einzelnen Stufen auf das Szintigramm können in den Abbildungen 2.11 und 2.12 abgelesen werden. Als mögliches Areal der Auftrefforte der $\gamma$-Quanten auf den Kristall ist ein gleichmäßiges Lini- 

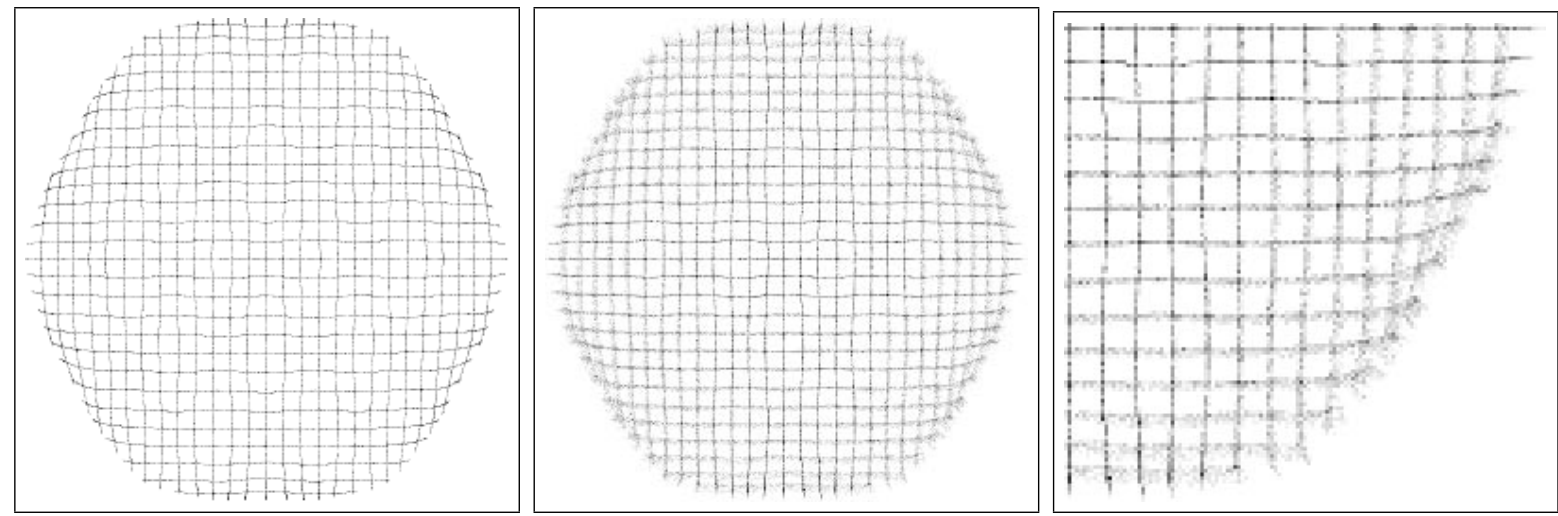

Abbildung 2.11: Simulierte Szintigramme für ein vorgegebenes Linienmuster. Links: feste Eindringtiefe der Quanten in den Kristall, keinerlei Rauschen. Mitte: wie links, nur mit zufälliger Eindringtiefe. Rechts: Ausschnitt aus dem mittleren Bild.

enmuster gewählt, um die Verzerrungen gut sichtbar zu machen. Ein Impulshöhenfenster ist nicht angewendet worden.In der Abbildung 2.11 stellt der linke Teil ein Szintigramm dar, daß erhalten werden könnte, wenn die $\gamma$-Quanten eine feste Eindringtiefe in den Kristall aufweisen würden und alle weiteren Prozesse rauschfrei wären. Reale $\gamma$-Quanten zeigen eine Verteilung der Eindringtiefen. Wird diese berücksichtigt, so führt dies allein schon zu einer unschärferen Abbildung aufgrund der in Abbildung 2.10 gezeigten Abhängigkeit der Photomultipliersignale von der Eindringtiefe, wie der mittlere und rechte Teil der Abbildung 2.11 zeigt. Der linke Teil der Abbildung 2.12 kann erhalten werden, wenn auch die
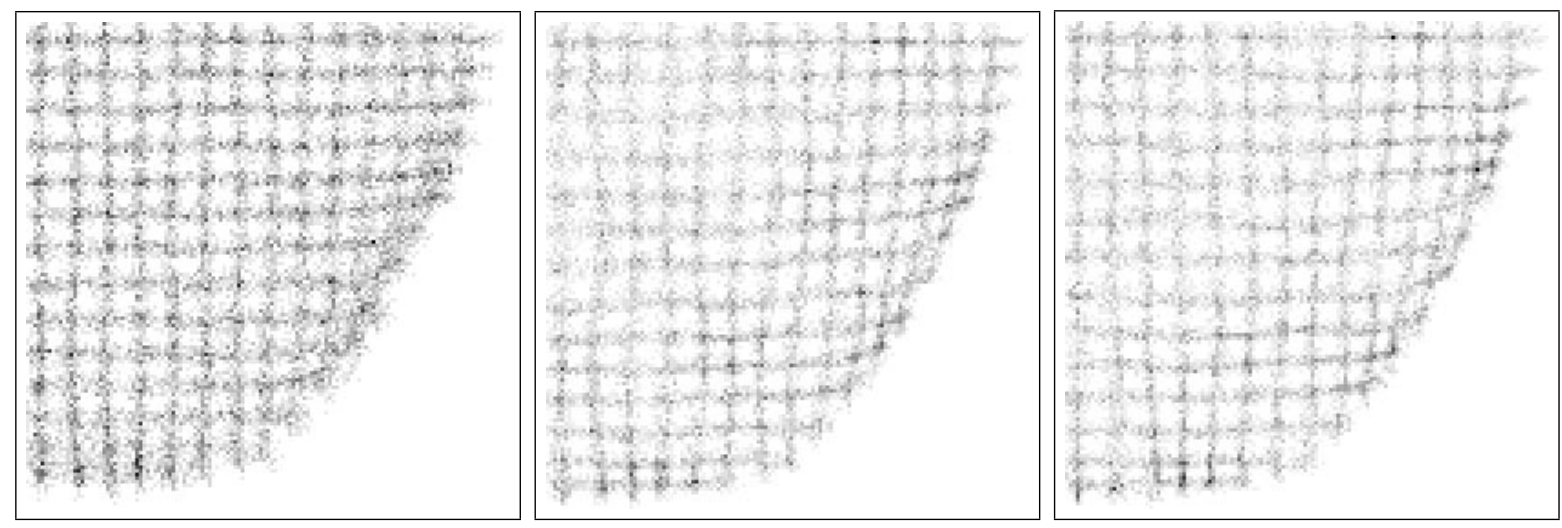

Abbildung 2.12: Wie Abbildung 2.11, jedoch mit eingestelltem Rauschen der Signale der einzelnen Photomultiplier.

einzelnen Signale der Photomultiplier mit einem Rauschen versehen werden. Der mittle- 
re und rechte Teil ist unter Verwendung zweier unterschiedlich hoher Schwellenwerte bei der Impulsarithmetik berechnet worden, d.h. ein Photomultipliersignal wird nur dann als von Null verschieden angenommen und trägt zur Impulsarithmetik bei, wenn es einen bestimmten Mindestwert übersteigt. Man kann erkennen, daß dies zu einer Verbesserung der Bildschärfe, vor allem in den Randbereichen führt.

Die mit Hilfe der Simulation der Abbildung einer Gammakamera erstellten Szintigramme zeigen den realen Szintigrammen sehr ähnliche Eigenschaften auf. Daher ist es mit der Simulation möglich, daß Verfahren zu Korrektion der Abbildung mit einer Gammakamera mit Hilfe einer Feinnadelstrahlquelle, wie es im folgenden Kapitel 3 vorgestellt wird, zu testen. Das Ergebnis findet sich in 3.2 


\section{Korrektion}

Aus den im Kapitel 2 beschriebenen Grundlagen über die Funktionsweise einer Gammakamera wird eine grundlegende Eigenschaft deutlich: die Abbildung ist inhärent nichtlinear. Diese Eigenschaft ist mit Hilfe technischer Verbesserungen zwar zu mildern, jedoch nicht auszuschließen. Jede Kamera, die nach dem Angerschen Prinzip gebaut ist, bedarf einer Korrektion.

Man kann ganz allgemein bei allen gängigen Verfahren zur Behebung der Abbildungsfehler drei Korrektionsschritte unterscheiden:

- Die Impulshöhenkorrektion [8] sollte die örtliche Variation des Summensignals aller Photomultiplier - das Energieäquivalent - so gut wie möglich eliminieren. Diese Korrektion ist sehr bedeutend für die Qualität des korrigierten Bildes, weil zur Eliminierung gestreuter Quanten ein Impulshöhenfenster darüber entscheidet, ob ein registriertes Quant bildwirksam wird.

- Die Linearitätskorrektion berichtigt die Verzerrungen der nichtlinearen Abbildung[30]. Auf diese Weise beseitigt diese Korrektion gleichzeitig auch diejenigen Inhomogenitäten, die darauf zurückzuführen sind, daß unterschiedlichen Pixeln unterschiedlich große Flächen auf dem Detektor durch die Verzerrungen zugeordnet werden.

- Homogenitätskorrektionen [16, 33, 40, 36, 28] sorgen für die Berücksichtigung von unterschiedlichen örtlichen Nachweiswahrscheinlichkeiten. In den meisten Fällen werden durch diesen Korrektionsschritt aber auch andere Fehler, wie z.B. die örtliche Variation der Form der Impulshöhenverteilung subsumiert. Dieser Korrektionsschritt ist oft in seiner Auswirkung weniger drastisch, da die mit ihm verbundenen Ursachen kleinerer Ordnung im Vergleich zu den beiden vorhergehenden sind. Im Gegensatz zu den beiden vorher genannten Korrektionen wichtet die Homogenitätskorrektion die einzelne Detektion eines Ereignisses, wobei Wichtung bedeutet, daß 
an einem Detektionsort bei z.B. 100 tatsächlich detektierten Quanten so getan wird, als ob hier 110 detektiert wären, an einem anderen Ort jedoch nur z.B. 105.

Die Reihenfolge bei der Durchführung der Korrektion ist meist die gleiche wie in der obigen Aufzählung; zwingend ist das Vorausgehen der ersten beiden Schritte - Impulsund Linearitätskorrektion - vor der Homogenitätskorrektion.

Die Grundlage für diese Korrektionen bilden Aufnahmen von künstlich vorgegebenen Verteilungen jeweiliger Radionukleide. Die Parameter für die Korrektionsalgorithmen werden durch Vergleich von Soll- und Istsituation gewonnen. Unterstellt wird dabei, daß die Sollsituation genau bekannt ist. Leider ist dies nicht immer der Fall oder es ist zumindestens zweifelhaft.

Bei der Gammakamera mischen sich zwei Eigenschaften: Auf der einen Seite die lokale Variation der Detektionseigenschaften und auf der anderen Seite das relativ geringe örtliche Auflösungsvermögen. Die Detektion vorgegebener Verteilungen von Radionukleiden, die relativ große zusammenhängende örtliche Bereiche des Szintillationskristalls beleuchten, subsumiert zwangsläufig die variierenden Eigenschaften der Gammakamera in diesen Bereichen. Sie sind damit weniger gut geeignet, qualitativ hochwertige Daten zur Korrektion zu liefern.

Das geeignetste Eingangssignal, um die Antwort eines Systems zu bestimmen, ist ein $\delta$-Impuls. Für die hier vorliegende Fragestellung bedeutet dies, daß ein punktueller Strahl aus monoenergetischen Gammaquanten hergestellt werden muß. Nun lassen sich Gammaquanten in dem hier interessierenden Energiebereich nicht optisch fokussieren. Die einzige Möglichkeit, dem Ideal eines $\delta(x, y, E)$-Impulses nahe zu kommen, ist die Hintereinanderschaltung mehrerer Blenden. Diese lassen nur einen kleinen Teil eines Raumwinkels der isotrop ausgestrahlten $\gamma$-Quanten zu, mit der Folge, daß, je kleiner das Raumwinkelelement wird, ein immer kleiner werdender Anteil der ausgestrahlten Quanten genutzt werden kann. Wird als Quelle der Gammaquanten aktives Material benutzt ${ }^{1}$, so kann die erreichbare Aktivität pro Volumeneinheit (die sog. spezifische Aktivität) nicht beliebig gesteigert werden, und es wird immer ein Kompromiß zwischen Kollimation und Ausbeute gemacht werden müssen.

Im Folgenden wird in 3.1 das allgemeine Konzept der Korrektion mit Hilfe der Feinnadelstrahlquelle vorgestellt. Hier werden auch Fragen der praktischen Durchführbarkeit

\footnotetext{
${ }^{1}$ Röntgenröhren können prinzipiell zwar auch Photonen des diskutierten Energiebereichs abgeben, allerdings sind diese Geräte sehr teuer und zeigen bei solchen Energiebereichen einen hohen nicht akzeptablen Verschleiß.
} 
wie z.B. die Dauer des Verfahrens erläutert. In 3.1.1 wird der Aufbau der Feinnadelstrahlquelle erörtert und anschließend ihr Abbildungsverhalten in 3.1.2. Aus den Erkenntnissen dieser Abschnitte wird in den Abschnitten 3.1.3 und 3.1.4 eine dem Abbildungsverhalten der Gammakamera angepaßte Definition von Bildelementen dargestellt. Der Abschnitt 3.2 zeigt schließlich das Verfahren in der Anwendung.

\subsection{Das Konzept}

Die hier vorgestellte Korrektion der Gammakamera beruht auf der Grundlage von Daten, die aus einer sukzessiven punktuellen Vermessung des Detektors mit Hilfe der Feinnadelstrahlquelle gewonnen werden. Ein besonderes Merkmal der Vermessung ist, daß die einzelnen punktuellen Messungen zeitlich voneinander getrennt und damit überlagerungsfrei stattfinden. Die Feinnadelstrahlquelle steht mit ihrem Stahlengang senkrecht zur Detektorkristallebene. Die Gammaquanten treffen also in gleicher Weise wie bei Vorliegen eines Parallellochkollimators mit Parallellochbohrung auf den Kristall ${ }^{2}$.

Der gesamte Detektorkristall wird untersucht, indem die Meßpunkte den Kristall überdecken. Als Überdeckung ist ein regelmäßiges, äquidistantes Raster gewählt. Jede Position der Feinnadelstrahlquelle definiert einen Rasterpunkt. Jedem Rasterpunkt wird durch eine im Abschnitt 3.1.3 beschriebene Vorschrift genau ein Pixel im korrigierten Bild zugeordnet. Diese Vorschrift stellt den zentralen Punkt des ganzen Konzepts dar und ist nur mit Hilfe einer Feinnadelstrahlquelle zu realisieren. Will man diese Vorschrift in das Schema bestehend aus Impulshöhen-, Linearitäts- und Homogenitätskorrektion einordnen, so entspricht sie der Impulshöhen- und Linearitätskorrektion als gleichzeitig.

Die Rasterpunkte werden mit einem zweidimensionalen Index $(i, j)$ adressiert. Zu jeder Position der Feinnadelstrahlquelle bzw. zu jedem Rasterpunkt gehört eine Reihe von Koordinaten, die an dieser Stelle festgelegt werden müssen.

Zunächst gehört zu jedem Rasterpunkt $(i, j)$ ein Ortskoordinatenpaar $\left(x_{P}(i, j), y_{P}(i, j)\right)$, welches die Position der Feinnadelstrahlquelle in dem Koordinatensystem $K_{P}$ angibt, das zu der Verfahrmechanik gehört. Punkte innerhalb dieses Koordinatensystem werden im

\footnotetext{
${ }^{2}$ Bei Verwendung anderer Kollimatorgeometrien, z.B. eines Fanbeam-Kollimators ist das hier vorgestellte Verfahren streng genommen nicht mehr verwendbar, weil die $\gamma$-Quanten nicht mehr senkrecht in den Kristall eindringen. Allerdings stellt es immer noch im Vergleich zu den bestehenden Methoden die weitaus genauere Alternative dar. Darüber hinaus ist mit der Feinnadelstrahlquelle im Prinzip auch eine Vermessung des Kristalls, wie sie eine allgemeinere Bohrungsgeometrie fordert, möglich.
} 
folgenden mit einem $P$ gekennzeichnet. Der Abstand von Rasterpunkt zu Rasterpunkt bestimmt die (reale) Pixelkantenlänge im auf diese Weise korrigierten Szintigramm. Die im Rahmen dieser Arbeit durchgeführten Messungen sind alle mit einer Pixelkantenlänge von $1.1 \mathrm{~mm}$ gemacht worden.

Als Folge der Detektion eines $\gamma$-Quants erhält man von der Gammakamera Werte für die Ortskoordinaten und die Impulshöhe, die als Punkte im dreidimensionalen Koordinatensystem $K_{\gamma}$ der Gammakamera angesehen werden können. Punkte $\left(x_{\gamma}, y_{\gamma}, E_{\gamma}\right)$ im Koordinatensystem der Gammakamera werden im folgenden mit einem $\gamma$ als unteren Index gekennzeichnet werden. Tatsächlich werden die Werte für die Gammakamerakoordinaten aus einer Analog/Digital-PC-Karte gelesen. Da die Samplingtiefe jedoch bei 16 bit liegt, können der Wertevorrat für die Gammakamerakoordinaten in Bezug auf die Rasterung der Szintigramme als quasi kontinuierlich angesehen werden.

Die Bildelemente eines Szintigramms können ebenfalls mit Hilfe eines zweidimensionalen Index adressiert werden. Wie oben bereits erwähnt und im Abschnitt 3.1.3 genau ausgeführt, kann man eine Vorschrift angeben, die jedem Rasterpunkt ein Bildelement zuordnet. Diese Zuordnung kann in der Weise geschehen, das zum Rasterpunkt $(i, j)$ genau das Bildelement $(i, j)$ im Szintigramm gehört. Man kann die Entsprechung von Bildpixel und Rasterpunkt der Feinnadelstrahlquelle als Definition eines Pixels auffassen. Die definierenden Eigenschaften stellen die Mittelwerte $\bar{x}_{\gamma}(i, j), \bar{y}_{\gamma}(i, j)$ und $\bar{E}_{\gamma}(i, j)$ dar, die aus der Menge der $\gamma$-Quanten gebildet werden können, die aus der Feinnadelstrahlquelle an einer Position des äquidistanten Rasters stammen.

Abbildung 3.1:

Skizze der Impulshöhenkorrektur zu verschiedenen Orten auf dem Detektionskristalls. Die grau unterlegte Fläche unterhalb des Photopeaks kennzeichnet denjenigen Anteil von $\gamma$-Quanten, der innerhalb des Impulshöhenfensters liegt.

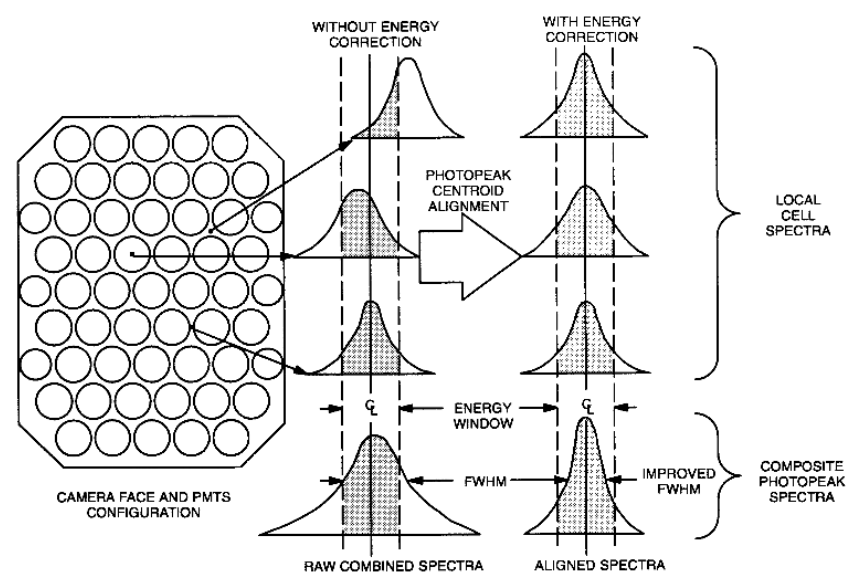

Die Korrektion der Impulshöhenwerte erfolgt ebenfalls von Rasterpunkt zu Rasterpunkt, indem der an einem Rasterpunkt $(i, j)$ gemessene Mittelwert $\bar{E}_{\gamma}(i, j)$ der Im- 
pulshöhe in Relation zu einem festzusetzenden Wert $E_{\text {const }}$ gesetzt wird. Dieser Wert $E_{\text {const }}$ entspricht dann demjenigen Wert der Impulshöhe, der der Energie des $\gamma$-Quants des jeweiligen Radionuklids zugeordnet wird. Rechnerisch wird der Quotient $q_{E}(i, j)=\frac{E_{\text {const }}}{\bar{E}_{\gamma}(i, j)}$ aus $E_{\text {const }}$ und dem Mittelwert $\bar{E}_{\gamma}(, j)$ und gebildet. Die Korrektion der Impulshöhe bei der Aufnahme eines Szintigramms erfolgt in der Art, daß jedes $\gamma$-Quant mit demjenigen Quotienten $q_{E}(i, j)$ multipliziert, der zu dem Rasterpunkt (i,j) gehört, dem dieses $\gamma$-Quant über die noch darzulegende Vorschrift zugewiesen wurde. Die Korrektion der Impulshöhe ist also eine multiplikative und entspricht in diesem Sinne der üblichen Vorgehensweise. Allerdings besteht hier ein genau definierter örtlicher Zusammenhang, da insbesondere keine Vermischung mit der mittleren Impulshöhe umliegender Detektorareale stattfindet. Wie wichtig dies ist, zeigt die Abbildung 3.1, in der deutlich gemacht wird, daß die effektive Breite des Photopeaks über den gesamten Detektorkristall umso kleiner ist, je besser die individuellen Photopeaks an bestimmten Stellen des Detektors in den richtigen $\mathrm{Zu}$ sammenhang zu den individuellen Photopeaks an anderen Stellen des Detektors gebracht werden.

Prinzipiell sind an dieser Stelle noch weitere Parameter zugänglich, die zu einer besseren Impulshöhendetektion verwendet werden können, wie z.B. die Schiefe und die Varianz der Impulshöhenverteilung nach multiplikativer Korrektion. Bei Verwendung eines Impulshöhenfensters sind dann die absoluten Lagen der Fenstergrenzen an diese Parameter anzupassen. In der Abb 3.2 ist der experimentelle Aufbau zur sukzessiven Vermessung

Abbildung 3.2:

Aufbau zur sukzessiven und punktuellen Vermessung des Detektionskristalls. Die Verfahrmechanik ist durch einen Plotter realisiert, wobei anstelle des Stiftes die Feinnadelstrahlquelle montiert ist.

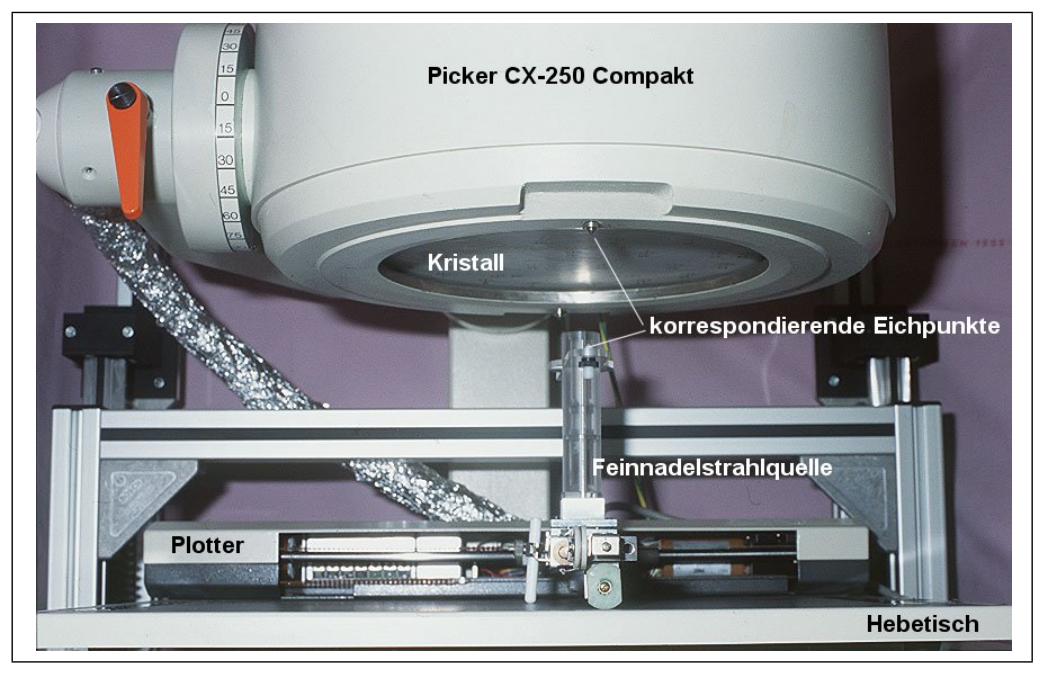

gezeigt. Bei der Durchführung der Vermessung ist der Abstand der Feinnadelstrahlquel- 
le zum Detektorkristall minimal, d.h. der Hebetisch mit der Verfahrmechanik befindet sich deutlich näher vor der Gammakamera.

Da die Vermessung aus Gründen, auf die im Abschnitt 3.1.1 noch genauer eingegangen wird, nicht in einem einzelnen Vorgang stattfinden kann, sind korrespondierende Eichpunkte an der Gammakamera und der Feinnadelstrahlquelle angebracht. Diese stellen einen reproduzierbaren eindeutigen Zusammenhang zwischen dem Koordinatensystem der Verfahrmechanik und der Gammakamera her. So ist es möglich, das Raster in Teilvermessungen abzufahren.

\subsubsection{Die Feinnadelstrahlquelle}

Ziel der Entwicklung der Feinnadelstrahlquelle war es, einen hochkollimierten Strahl von Gammaquanten zu erzielen, um damit einen möglichst kleinen und genau definierten Bereich des Detektionskristalls auszuleuchten. Daneben wurden die Dimensionen so gewählt, daß eine Verfahrbarkeit ohne großen Aufwand ermöglicht wurde Zu berücksichtigen war auch eine hinreichend große Ausbeute, um die erforderlichen Meßzeiten akzeptabel zu halten. Diese Erfordernisse haben zu folgender Form der Feinnadelstrahlquelle geführt.

Die Feinnadelstrahlquelle beinhaltet ein zylinderförmiges Behältnis aus Wolfram in das ein Hohlzylinder (Außendurchmesser $4 \mathrm{~mm}$ ) aus Teflon eingelassen ist. Dessen $30 \mathrm{~mm}$ lange Innenbohrung hat einen Durchmesser ${ }^{3}$ von $2 \mathrm{~mm}$. Es ergibt sich somit ein befüllbares Volumen von rund $90 \mathrm{~mm}^{3}$, das mit einer wässrigen Lösung des Nuklids gefüllt wird. Die Gammaquanten aus dem Reservoir werden durch ein System von drei Blenden aus Blei und Wolfram (die erste Blende) kollimiert. Der Innendurchmesser der Blenden selbst beträgt $1 \mathrm{~mm}$. Das Wolframbehältnis und die Bleiblenden sind in einen Zylinder aus Plexiglas als tragendes Element eingelassen. Der Abstand der Blenden voneinander ist nicht äquidistant, sondern für die Blendengeometrie (Gesamtdurchmesser der Blende und Bohrungslänge) optimiert. Die entsprechenden Maße sind der Abbildung $3.3 \mathrm{zu}$ entnehmen.

Die vorliegende Gestalt der Feinnadelstrahlquelle ist das Ergebnis ausgedehnter Simulationen [24]. Eine wichtige Erkenntnis aus diesen Simulationen ist, daß bei der Wahl der vorliegenden Materialien und der gegebenen Geometrie die Berechnungen bzgl. Ausbeute und Intensitätsverteilungen ohne große Fehler mit Hilfe der geometrischen Optik durchgeführt werden können. In diesem Zusammenhang ist die Tatsache interessant, daß

\footnotetext{
${ }^{3}$ Der Teflon-Einsatz wurde erst im Nachhinein eingepasst. In der ersten Version gab es nur einen Behälter aus Blei, der allerdings massive Probleme bereitete. Die Gründe dafür sind in dem Abschnitt 3.1.2 angeführt.
} 
Abbildung 3.3:

Planzeichnung der Feinnadelstrahlquelle. Alle Maße in Millimeter. Füllkörper und Blenden sind in mehrere tragenden Plexiglaszylinder eingelassen, die ihrerseits entlang zweier Stahlstäbe geführt werden. Die Stahlstäbe sind in einem Sockel fest verankert. Durch die modulare Bauweise sind unterschiedliche Strahlenganglängen realisierbar.

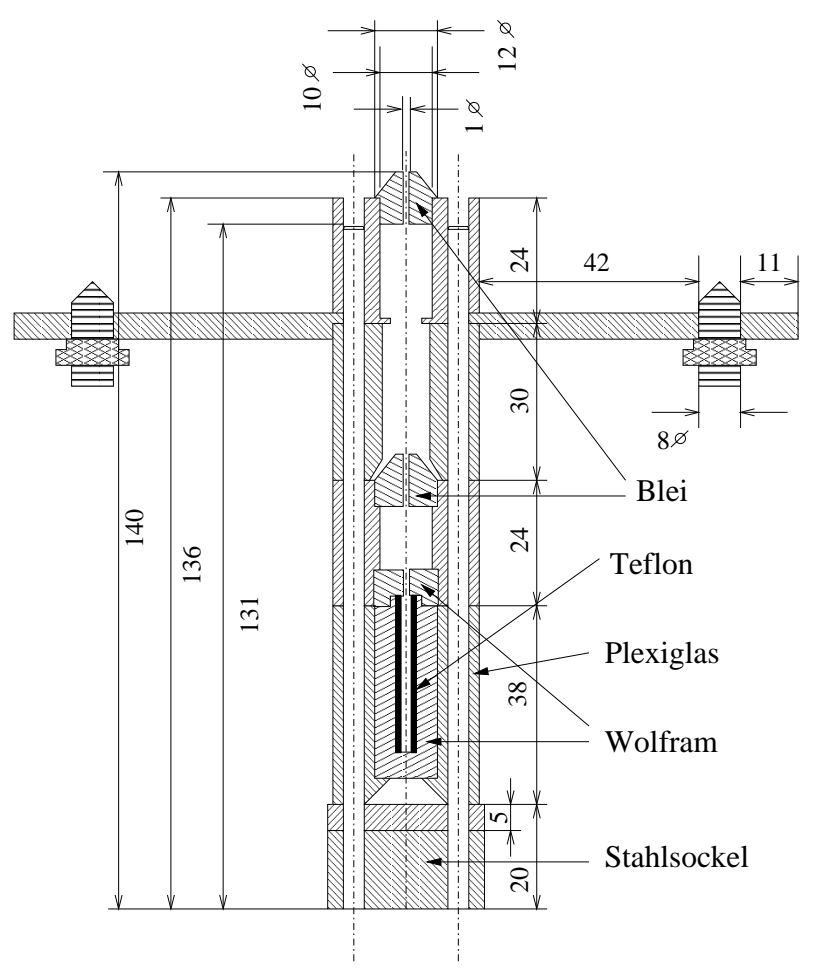

Abbildung 3.4:

Photo der realen Feinnadelstrahlquelle .

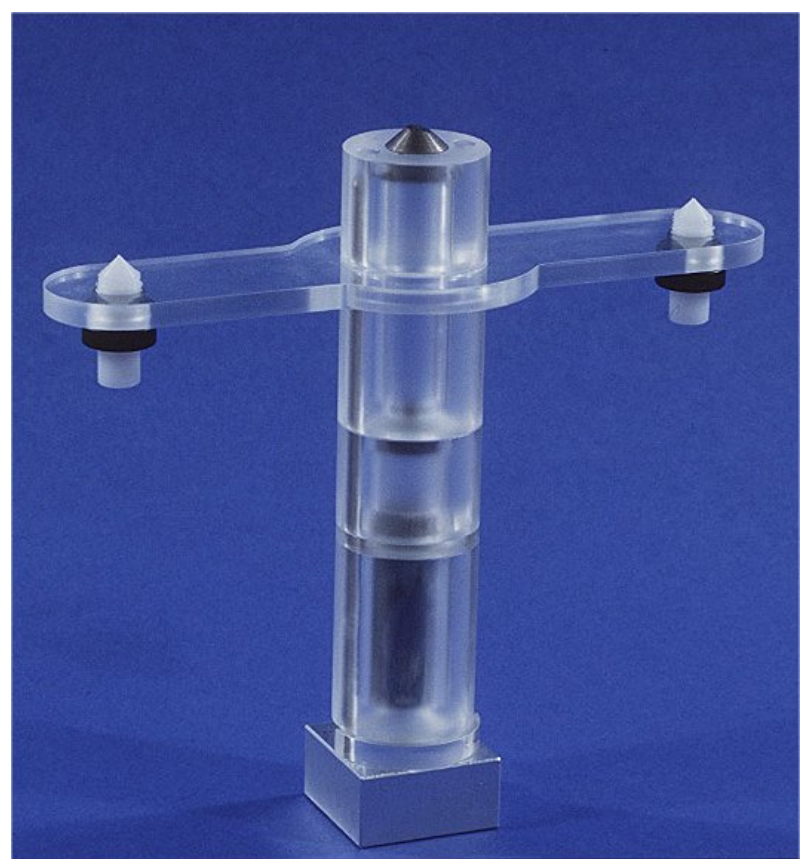


es kaum Compton-, dagegen jedoch einen nicht zu vernachlässigen Anteil an RayleighStreuung gibt. Da Rayleigh-Streuung elastisch ist, kann demnach davon ausgegangen werden, daß das Energie-Profil der austretenden Strahlung demjenigen der Strahlung ohne Wechselwirkung sehr ähnlich ist. Zudem treten bei der Rayleigh-Streuung zum überwiegenden Anteil nur kleine Streuwinkel auf, so daß z.B. die Ausbeute positiv beeinflußt wird, ohne daß die geometrisch-optische Näherung entscheidend schlechter wird.

Zur Berechnung der Ausbeute und Strahlaufweitung wurden drei ausgewählte Wege innerhalb des Strahlgangs herangezogen, die sich aus den jeweiligen Startpunkten der $\gamma$ Quanten im Vorratsbehälter ergeben: Oberer Rand $(85 \mathrm{~mm})$, Mitte $(100 \mathrm{~mm})$ und unterer Rand (115 mm) der Befüllung. Die Ergebnisse finden sich in Tabelle 3.5. Bei der Berechnung der Ausbeute ist zu beachten, daß nur ein Viertel der Gesamtbefüllung zur Ausbeute beiträgt, da die Bohrung des Behältnisses $2 \mathrm{~mm}$ beträgt.

\begin{tabular}{|c|c|c|c|}
\hline Startpunkt & Weglänge $[\mathrm{mm}]$ & Raumwinkel $[\Omega]$ & Strahlaufweitung $[\mathrm{rad}]$ \\
\hline Oberer Rand & 85 & $2.16 \cdot 10^{-6}$ & $5.88 \cdot 10^{-3}$ \\
\hline Mitte & 100 & $1.56 \cdot 10^{-6}$ & $5.00 \cdot 10^{-3}$ \\
\hline Unterer Rand & 115 & $1.18 \cdot 10^{-6}$ & $4.35 \cdot 10^{-3}$ \\
\hline
\end{tabular}

Abbildung 3.5: Kenngrößen der Feinnadelstrahlquelle.

Wie im Abschnitt 3.1erwähnt, wird die Feinnadelstrahlquelle zur sukzessiven punktuellen Vermessung des Detektionskristalls eingesetzt. Neben der rein geometrisch bedingten Ausbeute spielt die Halbwertszeit des verwendeten Radionuklids eine ganz entscheidende Rolle bei der Frage, wie viele Punkte auf dem Detektionskristall mit einer Befüllung vermeßbar sind. Darüber hinaus trat in der Durchführung der Messung ein weiterer Effekt auf, der die Ausbeute drastisch verschlechterte. Es handelt sich dabei um die Adsorption des Radionuklids an der Behältniswand. Da diese nicht im Strahlengang liegt, tragen die adsorbierten Radionuklide nicht mehr zur Ausbeute bei und verringern gleichzeitig die Konzentration zerfallsbereiter Kerne innerhalb des im Strahlengang liegenden Volumens.

\section{Ausbeute}

Zunächst soll die theoretisch zu erwartende Anzahl von Rasterpunkten berechnet werden, die mit einer Befüllung der Feinnadelstrahlquelle und jeweils gleicher mittlerer Anzahl von $\gamma$-Quanten pro Rasterpunkt aufgenommen werden können: Bei gegebener Startmeßdauer 
$t_{0}$ und einer vorgegebenen Maximalmeßdauer $t_{\max }$, die deutlich kleiner als die Halbwertszeit $t_{1 / 2}$ ist, kann die Anzahl der vermessenen Rasterpunkte wie folgt erhalten werden. Die kulminierte oder insgesamt aufgelaufene Meßdauer $t_{k}$ ergibt sich nach

$$
t_{\text {max }}=t_{0} \exp \left(\log (2) \frac{t_{k}}{t_{1 / 2}}\right)
$$

$\mathrm{zu}$

$$
t_{k}=\frac{t_{1 / 2}}{\log (2)} \log \frac{t_{\max }}{t_{0}}
$$

Die Anzahl der vermessbaren Rasterpunkte ist demnach

$$
n=\int_{0}^{t_{k}} \frac{1}{t_{0}} \exp \left(\frac{t}{t_{1 / 2}} \log (2)\right) d t=\frac{t_{1 / 2}}{t_{0} \log (2)}\left(1-\frac{t_{0}}{t_{\max }}\right)
$$
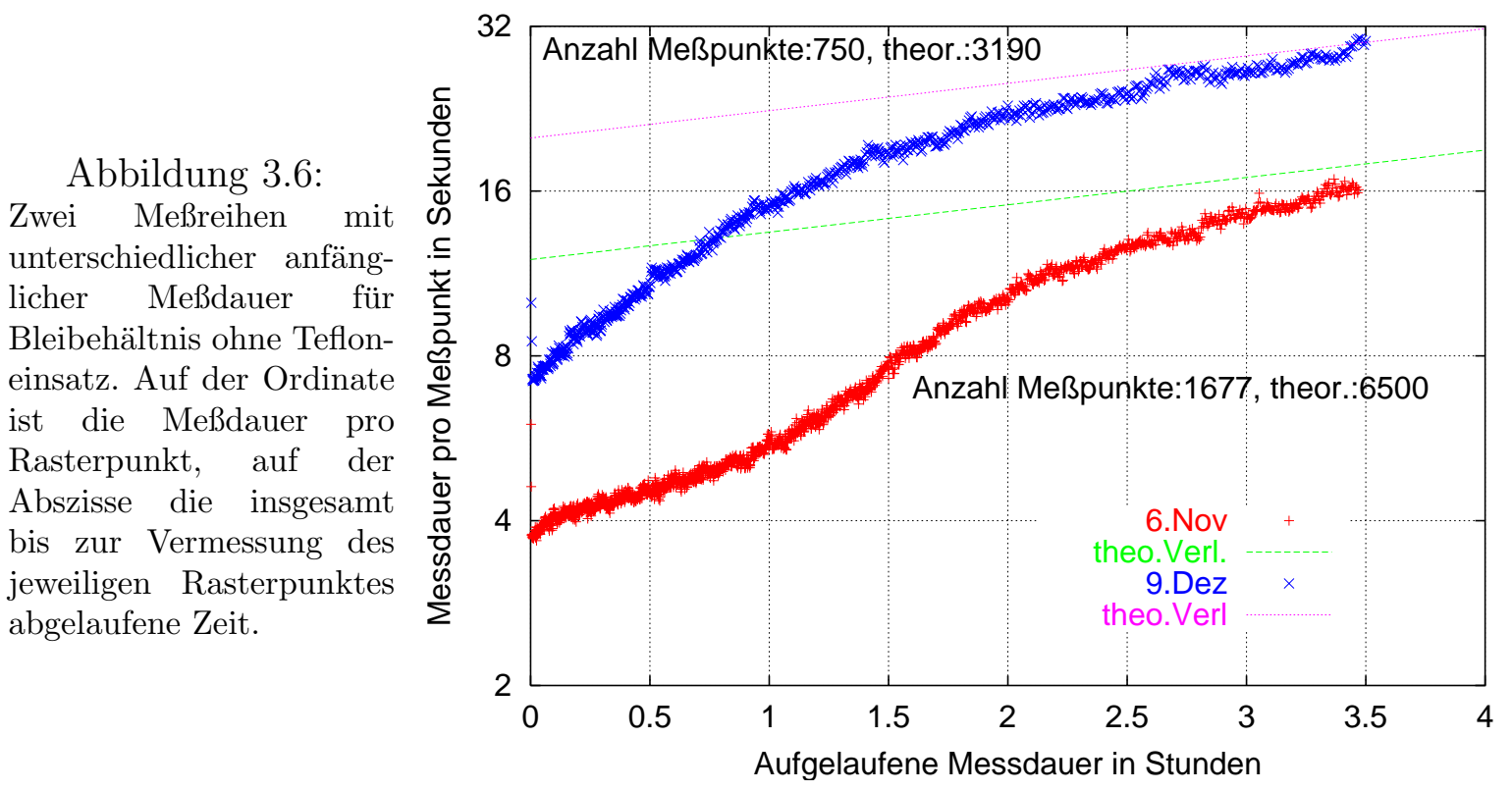

In der Abb. 3.6 sind zwei Meßreihen der Feinnadelstrahlquelle mit dem anfänglich verwendeten Bleibehältnis dargestellt. Aufgetragen ist auf der Abszisse die kulminierte Messdauer - also die bis zur Messung am aktuellen Meßpunkt insgesamt verstrichene Zeit -, auf der Ordinate die Meßdauer pro Meßpunkt. Der wesentliche Unterschied dieser beiden Meßreihen besteht in der deutlich unterschiedlichen Meßdauer für die ersten Werte: einmal knapp 4 und einmal knapp 8 Sekunden. 
Abbildung 3.7:

Meßreihe mit Tefloneinsatz in Wolframbehältnis, ansonsten wie Abbildung 3.6

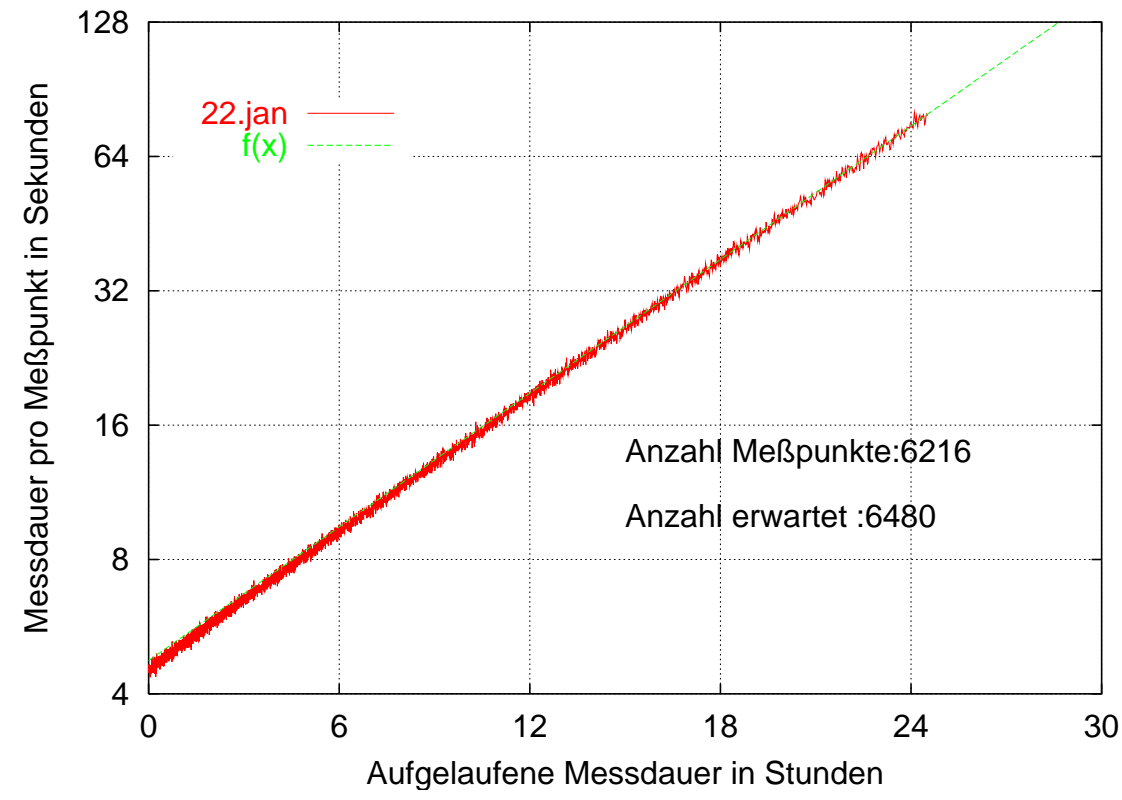

Wendet man die Gleichung (3.3) auf die in Abb. 3.6 gezeigten Meßreihen an, so heißt das, daß anstelle der 1677 bzw. 750 Meßpunkte 6500 bzw. 3190 theoretisch zu erwarten gewesen wären. Der Verlust ist dramatisch und macht eine Vermessung des gesamten Detektionskristalls in tragbarer Zeit unmöglich.

Zusätzlich zu den beiden Meßreihen sind die theoretisch zu erwartenden Kurven aufgetragen, die sich aus der Halbwertszeit des ${ }^{99 m}$ Tc ergeben. Dabei sind die Abzissenwerte so gewählt, daß die Meßreihen sich asymptotisch zu nähern scheinen. Deutlich ist zu erkennen, daß die scheinbare Halbwertszeit zu Anfang viel kürzer als der theoretisch zu erwartende Wert von 6.03 Stunden ist.

Der Verlauf der Meßdauer in Abb. 3.6 legt die Vermutung nahe, daß es sich um eine Adsorption des ${ }^{99 m}$ Tc an die Behältniswand aus Blei handelt. Das Phänomen der Adsorption ist in der Nuklearmedizin im Zusammenhang mit Phantomen durchaus bekannt und bereitet Schwierigkeiten bei der Bereitstellung von möglichst homogenen Aktivitätsverteilungen (Flächenphantome für Homogenitätskontrollen).

Um die Adsoprtion zu verhindern oder zumindestens auf ein tragbares Maß herabzusenken, bietet sich die Auskleidung des Behältnisses mit Teflon an, da dieses Material nur geringe Oberflächenadsorption zeigt. Als das Bleibehältnis durch ein mit Teflon ausgekleidetes Wolframbehältnis ersetzt wurde, hat sich dies auch im vorliegenden Fall, wie Abbildung 3.7 zeigt, bestätigt. 
Die typisch zu erzielenden Ausbeuten lagen zwischen 5000 und 10000 vermessenen Rasterpunkten pro Befüllung, so daß für die notwendigen 51500 Meßpunkte $^{4}$ etwa 7 Meßreihen notwendig waren. Da an zwei Tagen pro Woche Pertechnetatlösung zur Verfügung stand, bedeutet dies, daß in einem Zeitraum von 3-4 Wochen der gesamte Kristall vermessen werden konnte, wobei eine einzelne Messung sich nie über wesentlich mehr als 24 Stunden ausgedehnte.

\subsubsection{Die Abbildung der $\gamma$-Quanten aus der Feinnadelstrahlquelle durch die Gammakamera}

Wie im Kapitel 2 beschrieben, sind viele an der Detektion der Gammaquanten beteiligten Prozesse stochastischer Natur. Das bedeutet, daß die zu einer festen Position der Feinnadelstrahlquelle von der Gammakamera registrierten $\gamma$-Quanten eine Stichprobe aus der Verteilung der szintigraphischen Koordinaten, die zu diesem Ort des Detektionskristall gehören, darstellt. Wie im nächsten Abschnitt 3.1.3 noch genauer ausgeführt, werden zur Korrektion der Abbildung die empirischen Mittelwerte dieser Verteilung herangezogen. Um eine Abschätzung der Güte der Korrektion zugrundeliegenden Parameter zu gewinnen, ist eine Abschätzung der Fehler der Mittelwerte anzugeben. Bei gegebener Verteilungsfunktion bedeutet dies eine Aussage über den Mindestumfang der Stichprobe. Es ist also zunächst zu prüfen, ob die erhaltenen Verteilungen einer bestimmten Verteilungsfunktion gehorchen.

Um Tests für die Verteilungsfunktion durchzuführen, ist die Feinnadelstrahlquelle an unterschiedlichen Stellen des Detektorkristalls positioniert worden. Die Auswahl dieser Positionen orientiert sich an den charakteristischen Positionen, die es im hexagonalen Photomulitplierarrays gibt. Diese sind in der Abbildung 3.8 skizziert.

Als erster Ansatz bietet sich die Gaußverteilung an. In der Abbildung 3.9 ist die Verteilung einer der beiden Ortskoordinaten, die $x$-Komponente, aufgetragen. Die gestrichelte Kurve stellt einen Fit mit einer Gaußfunktion,

$$
f\left(x_{\gamma}\right)=\frac{N}{\sqrt{2 \pi} \sigma} \exp \left(\left(\frac{\left(x_{\gamma}-\left\langle x_{\gamma}\right\rangle\right)^{2}}{2 \sigma^{2}}\right)\right)
$$

dar, wobei $\sigma^{2}$ die Varianz und $\left\langle x_{\gamma}\right\rangle$ den Mittelwert einer Gaußverteilung darstellt. Neben dem rein visuellen Eindruck, der, wie die Abbildung 3.9 verdeutlicht, zeigt, daß die

\footnotetext{
${ }^{4}$ Die Anzahl der Meßpunkte ergibt sich aus einem 256er Raster mit kreisförmigen Ausschnitt: $256^{2} \cdot \frac{\pi}{4}$
} 
Abbildung 3.8:

Auswahl von fünf charakteristischen Positionen im hexagonalen Photomultiplierarray aus 37 Photomultipliern der CX-250 Gammakamera. Die grau hinterlegten Kreise markieren die Position der Feinnadelstrahlquelle, die Mittelpunkte dieser Kreise stellen die Eintrittspunkte für die $\gamma$ Quanten dar.

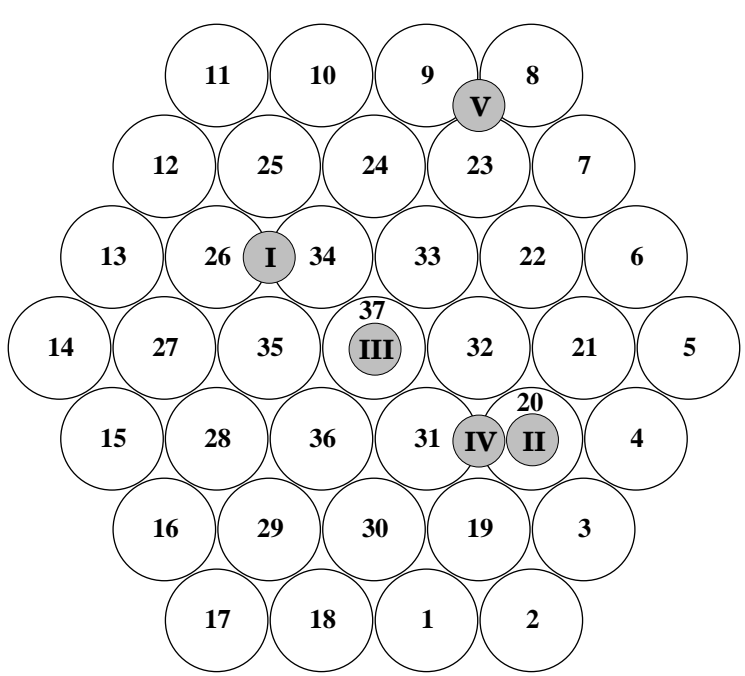

Verteilung in guter Näherung als Gaußfunktion beschrieben werden kann, wird dies auch durch einen $\chi^{2}$-Test nach Abzug des Untergrundes mit einer hohen Signifikanz von 0.993 bestätigt. Diese Aussage gilt ohne Einschränkung für die Testpunkte I - IV. Bei Testpunkt V zeigt die Verteilung eine nicht zu vernachlässigende Schiefe. Allerdings liegt dieser Testpunkt außerhalb des Gesichtsfeldes, welches der Korrektion zugänglich ist.

Abbildung 3.9:

Histogramm der Verteilung des Koordinatensignals für die Position IV. Aufgetragen ist die $\mathrm{x}-$ Komponente. Die Pfeile markieren die Breite der Verteilung auf halber Höhe des Maximums.

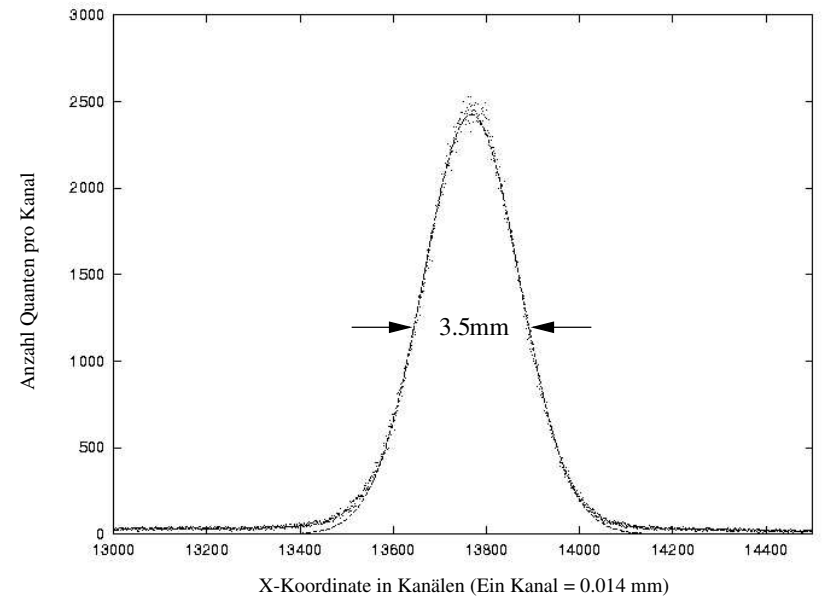

Für die mit der Feinnadelstrahlquelle gemachten Messungen an den Testpunkten liegen typische Werte für die empirische Standardabweichung der Ortskoordinate bei ungefähr 105 Kanälen bzw. rund 1.5 mm. Dies bedeutet für die Breite der Gaußverteilung bei halber 
Höhe des Maximums eine Größe von $3.5 \mathrm{~mm}$. Dies stimmt in der Größenordnung gut mit dem vom Hersteller angegebenen Aufösungsvermögen von etwa $4 \mathrm{~mm}$ überein.

Abbildung 3.10:

Verlauf der empirischen Varianz als Funktion des Stichprobenumfangs.

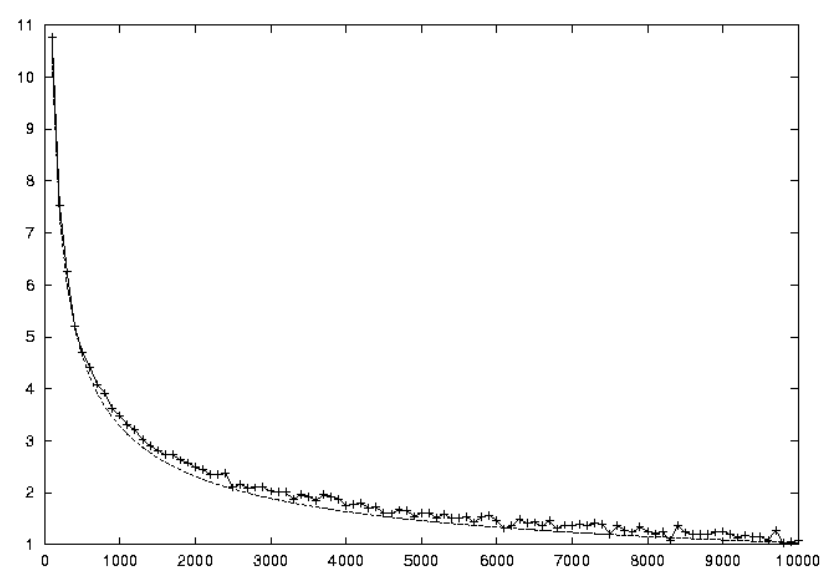

Bei Annahme einer zugrunde liegenden Gaußverteilung mit der Varianz $\sigma^{2}$, ist der Fehler $d m$ der Schätzung des Mittelwertes in Abhängigkeit vom Stichprobenumfang $N$ durch folgenden Ausdruck gegeben:

$$
d m=\frac{\sigma}{\sqrt{N}} \quad \Longrightarrow \quad N \geq\left(\frac{\sigma}{d m}\right)^{2}
$$

Dieser Zusammenhang kann ebenfalls experimentell überprüft werden. Das Ergebnis ist in Abb.3.10 zu sehen. Bei einer vorgegebenen mittleren Genauigkeit von mindestens \pm 3 Kanälen (entsprechend einer Genauigkeit von $0.042 \mathrm{~mm}$ ) kann aus der Abb.3.10 abgelesen werden, daß dazu etwa $1500 \gamma$-Quanten pro Stichprobe detektiert werden müssen. Tatsächlich sind für alle in dieser Arbeit verwendeten Messungen $1550 \gamma$-Quanten pro Rasterpunkt aufgenommen worden.

Im Falle der Impulshöhe ist neben dem Strahlungshintergrund der Verteilung auch noch ein nicht völlig zu unterdrückender Streuanteil aufgeprägt. Dennoch zeigt Abbildung 3.11, daß bei geeigneter Auswahl eine Mittelwertbildung weiterhin sinnvoll bleibt, weil der gestreute Anteil als klein angesehen werden kann. Die Feinnadelstrahlquelle stellt also auch im Falle der Impulshöhe ein geeignetes Instrument zur Untersuchung der Kameraeigenschaften in lokal aufgelöster Weise dar. Besonders bemerkenswert ist, daß in der Abbil- 
Abbildung 3.11:

Histogramm der Verteilung des Signals der Impulshöhenkoordinate, die zu einer festen Position der Feinnadelstrahlquelle gehört.

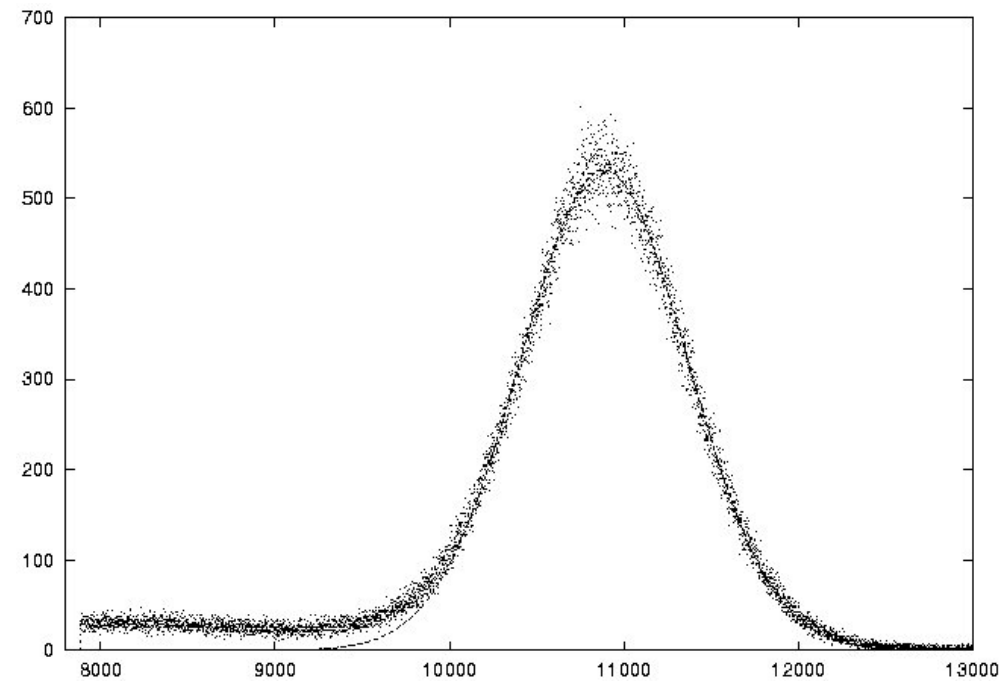

dung 3.11 kaum Comptonstreuung stattzufinden scheint, obwohl diese auch im Kristall selbst stattfindet und zu höheren Streuanteilen führen müßte wie man sie auch bei Aufnahmen räumlich ausgedehnter Radionuklidverteilungen auch ohne Streumaterial beobachtet (siehe z.B. das Impulshöhenspektrum in Abbildung 3.23 auf Seite 55). Die Ursache dafür, daß diese hier nicht beobachtet werden kann, liegt daran, daß man den räumlichen Bereich, in dem die $\gamma$-Quanten aus der Feinnadelstrahlquelle kommen können, bei Kenntnis ihrer Position stark einschränken kann. Ein großer Teil derjenigen $\gamma$-Quanten, die innerhalb des Detektionskristalls eine Comptonstreuung erfahren können deshalb aussortiert werden, weil sie durch die damit verbundene Verlagerung des Szintillationsschwerpunkts nicht mehr in dem erwarteten Bereich liegen. Sie tragen daher nicht zur Bildung des Impulshöhenspektrums 3.11 bei. Dies steigert die Qualität der Aussage über die mittlere Impulshöhenantwort und kann nur mit einer Feinnadelstrahlquelle erreicht werden.

\subsubsection{Pixel-Definition}

Wie im vorhergehenden Kapitel 3.1.2 gezeigt, liefert jede Messung einer Feinnadelstrahlposition prinzipiell gut definierte Verteilungen der $x_{\gamma}, y_{\gamma}$ und $E_{\gamma}$ Werte. Die Einschränkung „prinzipiell“soll auf den Umstand hinweisen, daß diese Verteilungen nur bei Unterdrückung von Störeinflüssen erhalten werden können. Da Störeinflüsse nicht vermieden werden können, müssen diese in geeigneter Weise herausgefiltert werden, damit die zu den Verteilungen gehörenden Mittelwerte zu der nun folgenden Definition eines Bildelements (Pixel) 
herangezogen werden können. Auf die konkrete Form des Filterns wird weiter unten eingegangen.

Jeder Rasterpunkt ist durch sechs Werte ausgezeichnet: Seinem Index $(i, j)$, den zugehören Koordinaten der Verfahrmechanik (Plotterkoordinatensystem) $x_{P}(i, j), y_{P}(i, j)$ und den aus der Messung erhaltenen Mittelwerten $\bar{x}_{\gamma}(i, j), \bar{y}_{\gamma}(i, j)$ und $\bar{E}_{\gamma}(i, j)$. Ein Bildraster aus Pixeln wird aufgebaut, in dem jedem Pixel $(i, j)$ der Rasterpunkt $(i, j)$ mit den dazugehörigen Werten zugeordnet wird. Zur Erstellung eines planaren Szintigramms, das aus diesen Pixeln besteht, und mit Hilfe einer rasterpunktweise vermessenen Gammakamera erstellt wird, kann folgende Vorschrift gegeben werden:

Ein von der Gammakamera primär bei den Koordinaten $x_{\gamma}$ und $y_{\gamma}$ detektiertes $\gamma$-Quant wird demjenigen Pixel $(i, j)$ zugeordnet, dessen zugehörigen Ortsmittelwerte $\bar{x}_{\gamma}(i, j)$ und $\bar{y}_{\gamma}(i, j)$ aus der rasterpunktweisen Vermessung den geringsten euklidischen Abstand $d$ aufweist.

$$
d=\sqrt{\left(\bar{x}_{\gamma}(i, j)-x_{\gamma}\right)^{2}-\left(\bar{y}_{\gamma}(i, j)-y_{\gamma}\right)^{2}} .
$$

Jedem dieser Bildelemente $(i, j)$ entspricht auf dem Detektorkristall ein Quadrat mit einer Kantenlänge entsprechend der Schrittweite von Rasterpunkt zu Rasterpunkt und einem Mittelpunkt, der der Position $x_{P}(i, j)$ und $y_{P}(i, j)$ der Feinnadelstrahlquelle entpricht.

Diese Vorschrift stellt die Definition für ein Pixel in der an die individuelle Gammakamera angepaßte Rasterung des dar. Angepaßt heißt, daß die Fläche $A_{\text {pix }}$ in der Ebene ${ }^{5}$ der Gammakamerakoordinaten, die einem Bildelement zugeordnet ist, variabel sein kann und sich nach den Eigenschaften der Abbildung der Gammakamera an diesem Ort richtet. Dies steht im Gegensatz zu der üblichen Vorgehensweise, in der zuerst ein in den Gammakamerakoordinaten äquidistantes Raster über die detektierten Orte gelegt wird und darauf folgend eine Entzerrung vorgenommen wird.

In der Literatur [17] ist ein dem hier vorgestellten ähnliches Verfahren vorgeschlagen worden, jedoch auf Grundlage einer flächenhaften Radionuklidverteilung, von der man annimmt, bei idealer Abbildung ein homogenes Szintigramm zu erzeugen. Die Ähnlichkeit der Verfahren begründet sich darin, daß in [17] solange die Grenzen der Bildelement

\footnotetext{
${ }^{5}$ Als Ebene der Gammakamerakoordinaten wird hier die Menge aller möglichen $x_{\gamma}$ und $y_{\gamma}$ Koordinatenpaare gesehen, die das Ergebnis der Detektion eines $\gamma$-Quants sind.
} 
verschoben werden, bis die Aufnahme homogen erscheint und somit ebenfalls Pixel unterschiedlicher Fläche aufweist. Die derart erreichte Linearität der Abbildung ist jedoch nicht befriedigend.

Beispielhaft ist die Form für ein willkürlich ausgewähltes Pixel in Abbildung 3.12 skizziert. Der Rand der Form ergibt sich aus den Mittelsenkrechten zu den Geraden, die vom dem Punkt $\left(\bar{x}_{\gamma}(i, j), \bar{y}_{\gamma}(i, j)\right)$ zu den nächsten acht Nachbarn gezogen werden können, weil die Mittelsenkrechte zur Verbindungslinie zwischen zwei Punkten die Menge derjenigen Punkte trennt, die jeweils zu einem der beiden Punkte den kürzesten euklidischen Abstand besitzen. Auf der Verbindungslinie liegen diejenigen Punkte, die zu beiden Punkten den gleichen Abstand besitzen. Kommt es zu dem seltenen Fall, daß ein $\gamma$-Quant genau auf eine solche Linie fällt, so erhalten beide Pixel einen Beitrag von 0.5 Quanten.

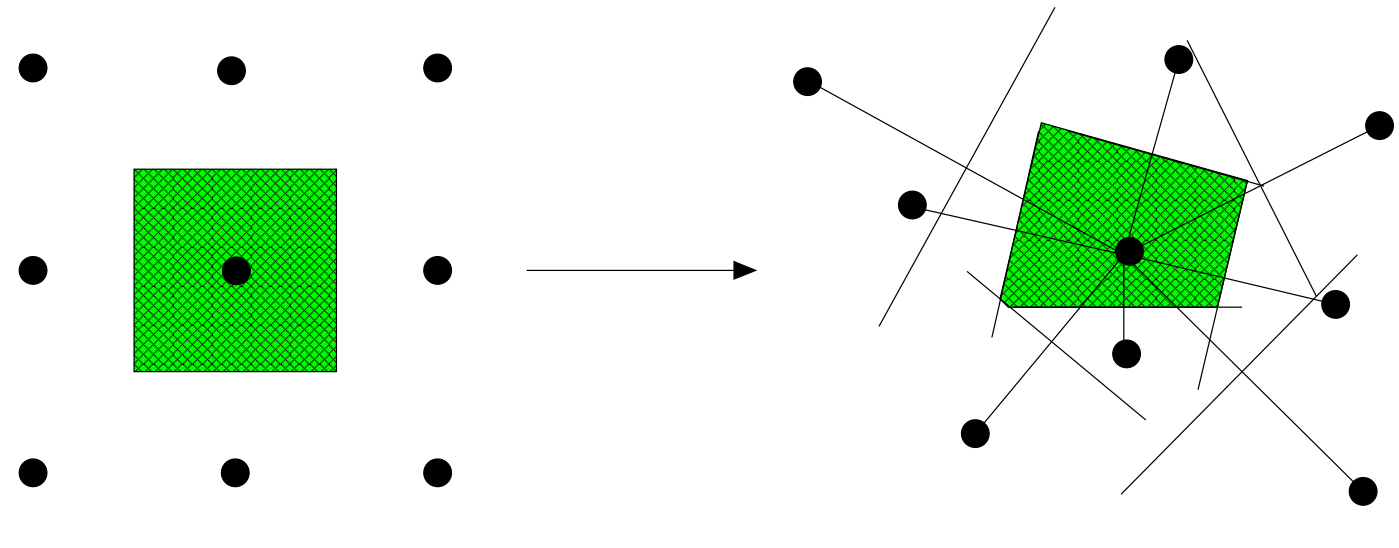

Abbildung 3.12:

Definition der Fläche in Gammakamerakoordinaten, die zu einem Rasterpunkt $(i, j)$ gehört.

Um die Entscheidung, zu welchem Pixel ein detektiertes $\gamma$-Quant gehört, in sinnvoller Weise treffen zu können, muß die interne Diskretisierung der Meßwerte in den szintigraphischen Koordinaten deutlich feiner sein, als das Rastermaß der aufzunehmenden Szintigramme. In dem Fall der vorliegenden Kamera konnte eine Rasterung von 256 • 256 der Szintigramme gewählt werden, da die interne elektronische Verarbeitung mit einer Diskretisierung von bis zu $2^{16} \cdot 2^{16}$ arbeitet.

Das Pixel $(i, j)$ wird durch die Mittelwerte $\bar{x} \gamma(i, j), \bar{y} \gamma(i, j)$ und $\bar{E} \gamma(i, j)$ repräsentiert, oder besser: definiert. Ein wichtiger Aspekt, der hierbei beachtet werden muß, ist die geeignete Wahl der bei der Berechnung der Mittelwerte zugrunde gelegten Stichprobe $V(i, j)$ aus der 
Verteilung der $\gamma$-Quanten. Die zunächst bei der Aufnahme eines einzelnen Rasterpunktes erhaltene Stichprobe $V_{0}(i, j)$ enthält noch Quanten, die nicht aus der Feinnadelstrahlquelle stammen ${ }^{6} . V_{0}(i, j)$ ist die Stichprobe aller detektierter Quanten, beinhaltet also Quanten aus dem gesamten, der Kamera zugänglichen Impulshöhenbereich $(\approx 50 \mathrm{keV}-$ $\approx 600 \mathrm{keV})$ und der gesamten Detektoroberfläche. Ursprung anderer Quanten als derjenigen aus der Feinnadelstrahlquelle ist z.B. die Hintergrundstrahlung. Zusätzlich können störende Einflüsse von Fremdaktivitäten stammen, da diese in einer nuklearmedizinischen Abteilung nicht auszuschließen sind und wurden während der Messungen immer wieder beobachtet. (siehe Bild 3.13, rote Linie).

Um die oben genannten Einflüsse weitgehend herauszufiltern, nutzt man die Tatsache, daß die Gammaquanten, die aus der Feinnadelstrahlquelle stammen, in einem relativ kleinen Areal verteilt sind. Der andere Teil der Strahlung verteilt sich hingegen über die gesamte Detektorfläche. Typische Zahlen in unserem Labor sind 300 bis 500 Quanten pro Sekunde über alle Impulshöhen oder 10 bis 15 pro Sekunde in einem Energiebereich von 100-180 keV . Die Picker CX-250 hat einen Durchmesser von 11 Zoll bzw. 27.94 cm. Somit ergibt sich eine flächenhafte Rate von $(0.016-0.024) \frac{1}{s \cdot \mathrm{cm}^{2}}$ im zuletzt genannten Energiebereich. Diese flächenhafte Rate ist sehr klein gegenüber derjenigen, die der hochkollimierte Strahl der Feinnadelstrahlquelle aufweist. Dies zeigt die folgende Abschätzung: Kommen nur $10 \gamma$-Quanten pro Sekunde aus der Feinnadelstrahlquelle, so ergibt sich eine flächenhafte Rate von etwa $1280 \frac{1}{s \cdot \mathrm{cm}^{2}}$ in dem Gebiet, welches durch die Quelle ausgeleuchtet wird.

Der große Unterschied in der flächenhaften Rate ist ein geeigneter Ansatzpunkt, um die Störeinflüsse wie folgt zu eliminieren: Legt man um den Mittelpunkt ein möglichst kleines Areal, daß jedoch groß genug ist, um möglichst alle aus der Feinnadelstrahlquelle stammenden Quanten zu berücksichtigen, so kann der Einfluß der Hintergrundstrahlung und anderer Einflüsse sehr gering gehalten werden. Die Lage des Areals und damit die Auswahl derjenigen $\gamma$-Quanten, die mit großer Wahrscheinlichkeit aus der Feinnadelstrahlquelle selbst stammen, kann iterativ bestimmt werden.

Die Stichprobe $V_{0}(i, j)$ wird herangezogen, um eine erste Schätzung für die drei Werte $\bar{x}_{\gamma}^{0}(i, j), \bar{y}_{\gamma}^{0}(i, j)$ und $\bar{E}_{\gamma}^{0}(i, j)$ zu erhalten. Um die großen Unterschiede in der flächenhaften Rate zu nutzen, werden in der ersten Iteration nicht die Mittelwerte aus der Stichprobe

\footnotetext{
${ }^{6} \mathrm{Zu}$ beachten ist, daß der Kristall offen ist. Während einzelnen Aufnahmen der Positionen der Feinnadelstrahlquelle ist kein Kollimator montiert, somit ist die Gammakamera Störungen relativ ungeschützt ausgeliefert
} 
$V_{0}(i, j)$ berechnet, sondern es wird ein Histogramm der Dichte der Verteilung gebildet und die Lage des Maximums innerhalb des Histogramms als erste Schätzung für die Mittelwerte angesehen. Dies ist sinnvoll, da das Maximum viel unempfindlicher gegen Störeinflüsse ist und bei einer Gaußverteilung mit dem Mittelwert zusammenfällt. Im Gegensatz zur einfachen Mittelwertbildung können so auch bei relativ hohem Hintergrund sinnvolle Schätzwerte sicher gefunden werden. Um diese Werte aus der ersten Schätzung herum werden Intervalle geeigneter Größenordnung $\delta_{0} x_{\gamma}, \delta_{0} y_{\gamma}$ und $\delta_{0} E_{\gamma}$ gelegt. Aus der Stichprobe $V_{0}(i, j)$ werden diejenigen Quanten verworfen, die nicht innerhalb der Intervalle $\bar{x}_{\gamma}^{0}(i, j) \pm \delta_{0} x_{\gamma}$ etc. liegen. Das Ergebnis ist eine neue Stichprobe $V_{1}(i, j)$, die eine Untermenge der Stichprobe $V_{0}(i, j)$ darstellt:

$$
V_{1}(i, j)=\left\{\begin{array}{rlrl}
x_{\gamma} & x_{\gamma} & \in\left[\bar{x}_{\gamma}^{0}(i, j)-\delta_{0} x_{\gamma}, \bar{x}_{\gamma}^{0}(i, j)+\delta_{0} x_{\gamma}\right] \\
y_{\gamma} \in V_{0}(i, j): & y_{\gamma} \in\left[\bar{y}_{\gamma}^{0}(i, j)-\delta_{0} y_{\gamma}, \bar{y}_{\gamma}^{0}(i, j)+\delta_{0} y_{\gamma}\right] \\
E_{\gamma} & E_{\gamma} \in\left[\bar{E}_{\gamma}^{0}(i, j)-\delta_{0} E_{\gamma}, \bar{E}_{\gamma}^{0}(i, j)+\delta_{0} E_{\gamma}\right]
\end{array}\right\}
$$

Mit Hilfe dieser Stichprobe $V_{1}(i, j)$ können nun echte Mittelwerte $\bar{x}_{\gamma}^{1}(i, j), \bar{y}_{\gamma}^{1}(i, j)$ und $\bar{E}_{\gamma}^{1}(i, j)$ berechnet werden. Um diese können im folgenden wieder neue Intervalle gelegt werden, wobei die Intervallgröße bis zu einem festzulegenden Minimum abnimmt $\left(\delta x_{n+1} \leq\right.$ $\left.\delta x_{n}\right)$. Das Verfahren wird iterativ fortgeführt und bricht ab, wenn die Änderung der Mittelwerte von Schritt zu Schritt klein genug ist. Natürlich ist es immer die ursprüngliche Stichprobe $V_{0}(i, j)$ aus der diejenigen Quanten ausgewählt werden, die zu einer neuen Stichprobe $V_{k}(i, j)$ beitragen:

$$
V_{k+1}(i, j)=\left\{\begin{aligned}
x_{\gamma} & x_{\gamma} \in\left[\bar{x}_{\gamma}^{k}(i, j)-\delta_{k} x_{\gamma}, \bar{x}_{\gamma}^{k}(i, j)+\delta_{k} x_{\gamma}\right] \\
y_{\gamma} \in V_{0}(i, j): y_{\gamma} & \in\left[\bar{y}_{\gamma}^{k}(i, j)-\delta_{k} y_{\gamma}, \bar{y}_{\gamma}^{k}(i, j)+\delta_{k} y_{\gamma}\right] \\
E_{\gamma} & E_{\gamma} \in\left[\bar{E}_{\gamma}^{k}(i, j)-\delta_{k} E_{\gamma}, \bar{E}_{\gamma}^{k}(i, j)+\delta_{k} E_{\gamma}\right]
\end{aligned}\right\}
$$

Diese Vorgehensweise ist selbstverständlich nur dann sinnvoll, wenn der Einfluß der Störstrahlung nicht zu groß ist. Vor allem darf die Störstrahlung keine ähnlich punktuelle Verteilung auf dem Detektor aufweisen wie die der Feinnadelstrahlquelle.

Wie gut dieses Verfahren in der Praxis auch bei Vorhandensein von Störstrahlung arbeitet, soll an der Schätzung der notwendigen Meßdauer für die jeweils nächste Position der Feinnadelstrahlquelle aus der Meßdauer für die aktuellen Positionen gezeigt werden. Diese dynamische Adaption der Meßdauer von Rasterpunkt zu Rasterpunkt ist notwendig, da, wie in 3.1.1 dargelegt, eine Abschätzung aus der Halbwertszeit des ${ }^{99 m} \mathrm{Tc}$ nicht möglich ist. Trotzdem muß, damit jeder Rasterpunkt mit etwa der gleichen statistischen 
Güte erhalten werden kann, garantiert werden, daß im Mittel immer die gleiche Anzahl $\gamma$-Quanten aus der Feinnadelstrahlquelle erhalten werden.

Abbildung 3.13:

Dynamische Adaption der Meßdauer und Filtern der Ereignisse. Die rosafarbene Kurve zeigt den Verlauf der jeweils von Punkt zu Punkt geschätzten Meßdauer. Zu diesen Meßdauern gehören dann die rote Kurve als Anzahl aller detektierter Quanten, die grüne Kurve zeigt den Umfang der Stichprobe $V_{1}$ und die blaue Kurve zeigt die Anzahl der verwendeten Quanten nach dem letzten Iterationsschritt an.

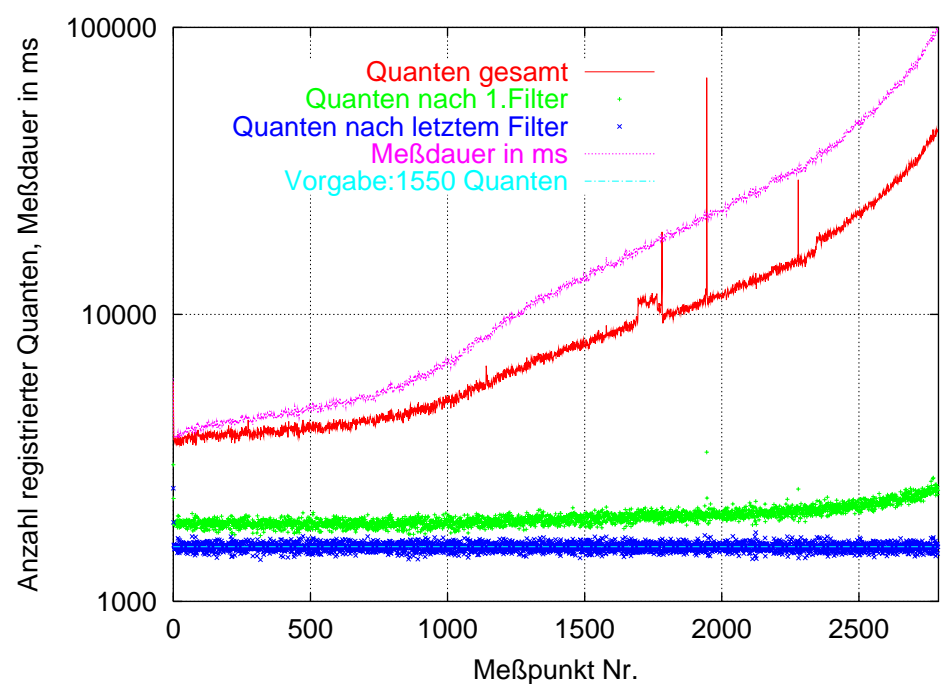

Die Abbildung 3.13 zeigt deutlich, wie trotz des unvorhersehbaren Verlaufs der Halbwertszeit - die Messung ist noch mit der alten Feinnadelstrahlquelle mit Bleitopf und ohne Tefloneinsatz gemacht - und deutlicher Störeinflüsse (delta und treppenförmige Erhöhung der Gesamtzahl registrierter Quanten, siehe rote Linie) durch zeitweilige externe Strahlung, eine hohe Konstanz der Anzahl verwertbarer $\gamma$-Quanten gegeben ist. Diese sind durch die blaue Linie gekennzeichnet und stellen die Anzahl der in der Verteilung $V_{k+1}(i, j)$ des letzten (Filter-) Iterationsschritts enthaltenden $\gamma$-Quanten. Der Mittelwert dieser Anzahl in der Abbildung 3.13 beträgt 1549.14, liegt also sehr gut in der Nähe der Vorgabe von 1550. Die Standardabweichung liegt mit 48.08 etwas über der theoretisch für eine Poissonverteilung mit Mittelwert $1550 \mathrm{zu}$ erwartenden Standardabweichung von 39.37. Diese Abweichung ist dadurch zu erklären, daß die Meßdauer selbst eine Schätzgröße darstellt und ebenfalls statistischen Schwankungen unterliegt und damit die Standardabweichung vergrößert.

Ebenfalls ist in der Abbildung 3.13 zu erkennen, daß die Störeinflüsse keinen wesentlichen Einfluß auf die Schätzung der Meßdauer (rosafarbene Linie) zeigen, diese also insbesondere keine Sprünge aufweist, die zu den Sprüngen der über den gesamten Kristall und alle Impulshöhen aufgelaufenen $\gamma$-Quanten (rote Linie) korrelieren. 

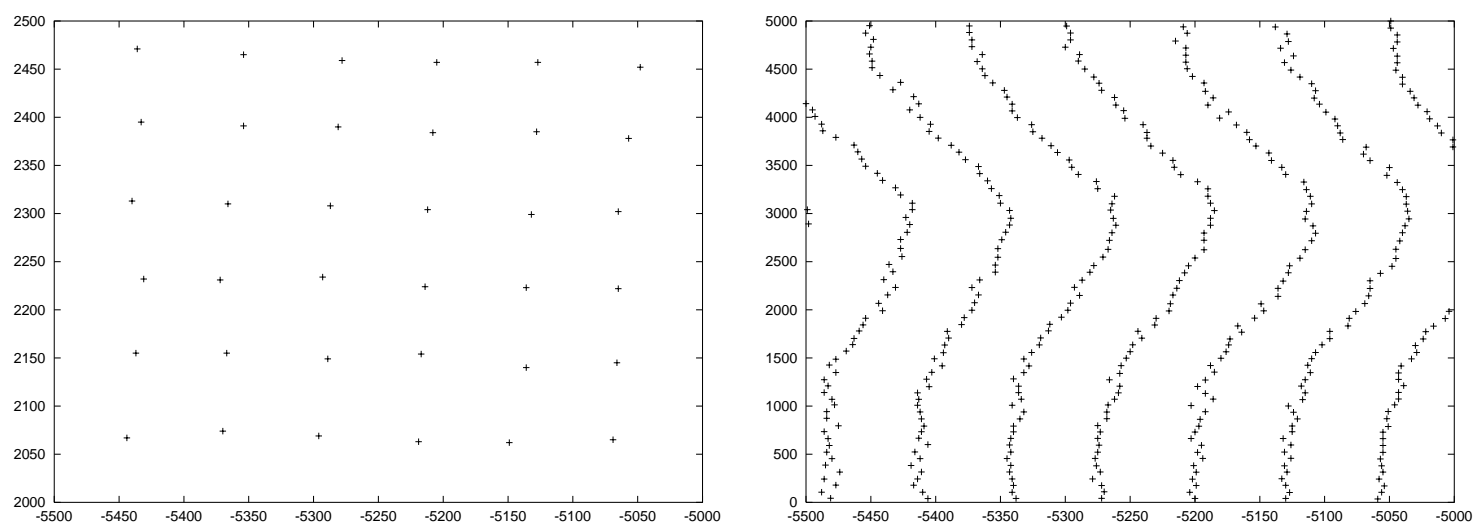

Abbildung 3.14: Zwei Ausschnitte aus dem Pixelraster. Im linken Teil der Abbildung sind die Maßstäbe beider Achsen gleich, im rechten ist die vertikale Achse dagegen stark gestaucht. Die auf den Achsen benutzten Einheiten entsprechen 0.014mm.

\subsubsection{Das Pixelraster}

Ist der gesamte Kristall vermessen, so ist das Ergebnis ein den Kristall überdeckendes Netz von Punkten, die nach obiger Definition für jeweils ein Pixel stehen. Aufgrund der Form (hervorstehende Ränder, siehe Abb. 3.2,Seite 31) der uns zur Verfügung stehenden CX250 Gammakamera ist es nicht möglich, wirklich den vollständigen Kristall abzufahren, jedoch ist der Bereich, der tatsächlich vermessen werden kann, größer als der üblicherweise benutzte und als hinreichend frei von Randeffekten ansehbare Bereich. In der Abbildung 3.14 sind zwei Ausschnitte mit unterschiedlichen Maßstäben für die Mittelwerte der Ortskoordinaten gezeigt, wie sie aus der vorgestellten iterativen Vorschrift resultieren. Die auf den Achsen aufgetragene Einheit ist immer $0.014 \mathrm{~mm}$. Im linken Teil der Abbildung sind die Maßstäbe für beide Achsen gleich und der Bildausschnitt entspricht einem Areal von $7 x 7$ mm. Da die Pixelkantenlänge $1.1 \mathrm{~mm}$ beträgt, sind erwartungsgemäß 6x6 Pixelmittelpunkte zu erkennen.

Im rechten Teil dagegen ist zur Verdeutlichung der Verzerrungen der Maßstab der vertikalen Achse um den Faktor 10 gestaucht. Dargestellt ist also ein Gebiet von $7 \mathrm{~mm} \times 70$ mm. Bei einem Durchmesser von $50.8 \mathrm{~mm}$ pro Photomultiplier entspricht der vertikale Auschnitt fast drei Radien. Dieser Teil der Abbildung zeigt, daß die Rasterpunkte nicht überall derart glatt verteilt sind, wie man es aus den Betrachtungen im Abschnitt 3.1.2 zu erwarten hat. Dies an den Bahnen parallel zur Ordinate zu erkennen, die teilweise Sprünge aufweist, die in dieser Ausprägung sicherlich nicht einer statistischen Schwankung bei der Mittelwertbildung zugeordnet werden kann, da diese überall und nicht nur Abschnittweise 


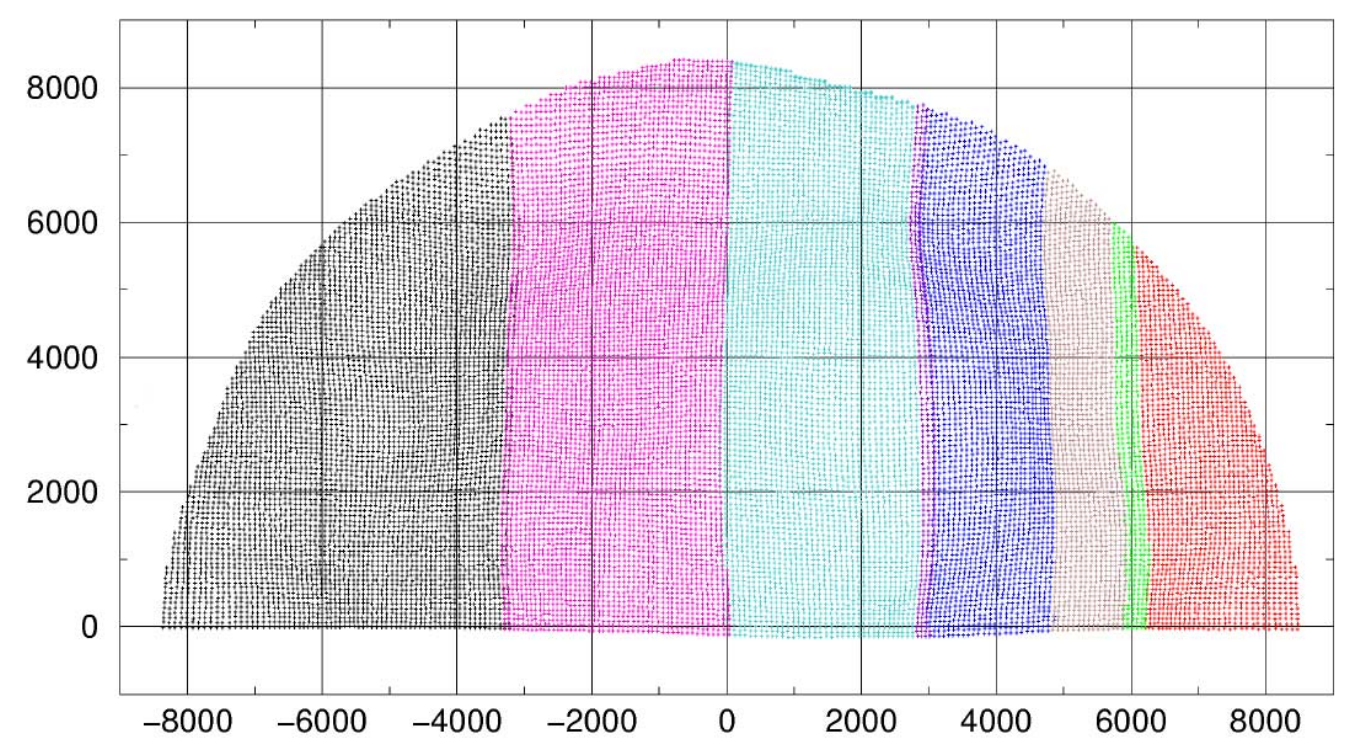

Abbildung 3.15: Rasterpunkte im Koordinatensystem der Gammakamera. Die unterschiedlichen Farben kennzeichnen unterschiedliche Meßreihen.

wirksam wäre. Die Ursache dieser Sprünge liegt in der experimentellen Realisation der Verfahrbarkeit der Feinnadelstrahlquelle durch einen handelsüblichen Plotter begründet. Das Gewicht der Feinnadelstrahlquelle überschreitet die zulässige Last der Verfahrmechanik, so daß es zu diesen kleinen Fehlpositionierungen $(\leq 0.15 \mathrm{~mm})$ kommt, die über dem vom Hersteller des Plotters angegebenen Maß $(\leq 0.07 \mathrm{~mm})$ liegen, bleiben allerdings so klein, daß das Verfahren als solches nicht in Frage gestellt wird. Darüber hinaus ist das Problem mit einer angemesseneren, stabileren Verfahrmechanik zu lösen.

In der Abbildung 3.15 ist das Pixelraster für die Hälfte des Kamerakopfes gezeigt und hat entsprechend der Kamerakopfform der CX-250 die Gestalt eines Halbkreises. Die unterschiedlichen Farben der Punkte der Ortsmittelwerte sind unterschiedlichen Teilmessungen zugeordnet, weil eine vollständige Messung mit einer Befüllung der Feinnadelstrahlquelle nicht möglich ist. Mit Hilfe der Eichpunkte (siehe Abb. 3.2) an Kamerakopf und Feinnadelstrahlquelle können die Teilmessungen wieder zusammengeführt werden. Die einzelnen Teilmessungen decken unterschiedliche große Flächen ab; zum einen, weil diese Messungen noch mit dem Bleibehältnis ohne Tefloneinsatz der Feinnadelstrahlquelle gemacht worden sind, zum anderen, weil unterschiedliche Startkonzentrationen vorlagen.

Die Verzerrungen zeigen eine typische tonnenförmige Gestalt: Dort, wo die Antwort 
des nächstgelegenen Photomultipliers überproportional hoch ist, werden die Punkte zusammengeschnürt, dort wo die entsprechende Antwort zu gering ist, werden die Punkte auseinandergezogen. Ebenfalls gut zu erkennen sind die Verzeichnungen in Randnähe der Gammakamera.

Im Laufe der Anfertigung dieser Arbeit ist es zum Bruch des Detektionskristalls gekommen, wahrscheinlich aus Gründen starker Temperaturschwankungen. Der Bruch verläuft fast durch den Mittelpunkt des Detektionskristalls. Optisch verhält sich dieser Bruch als teildurchlässiger Spiegel an Grenzschichten, wie man es auch von gesplissenem Glas kennt. Das Szintillationslicht wird an der Bruchstelle zum überwiegenden Anteil reflektiert und trennt somit die Detektorgebiete links und rechts des Bruchs optisch voneinander.
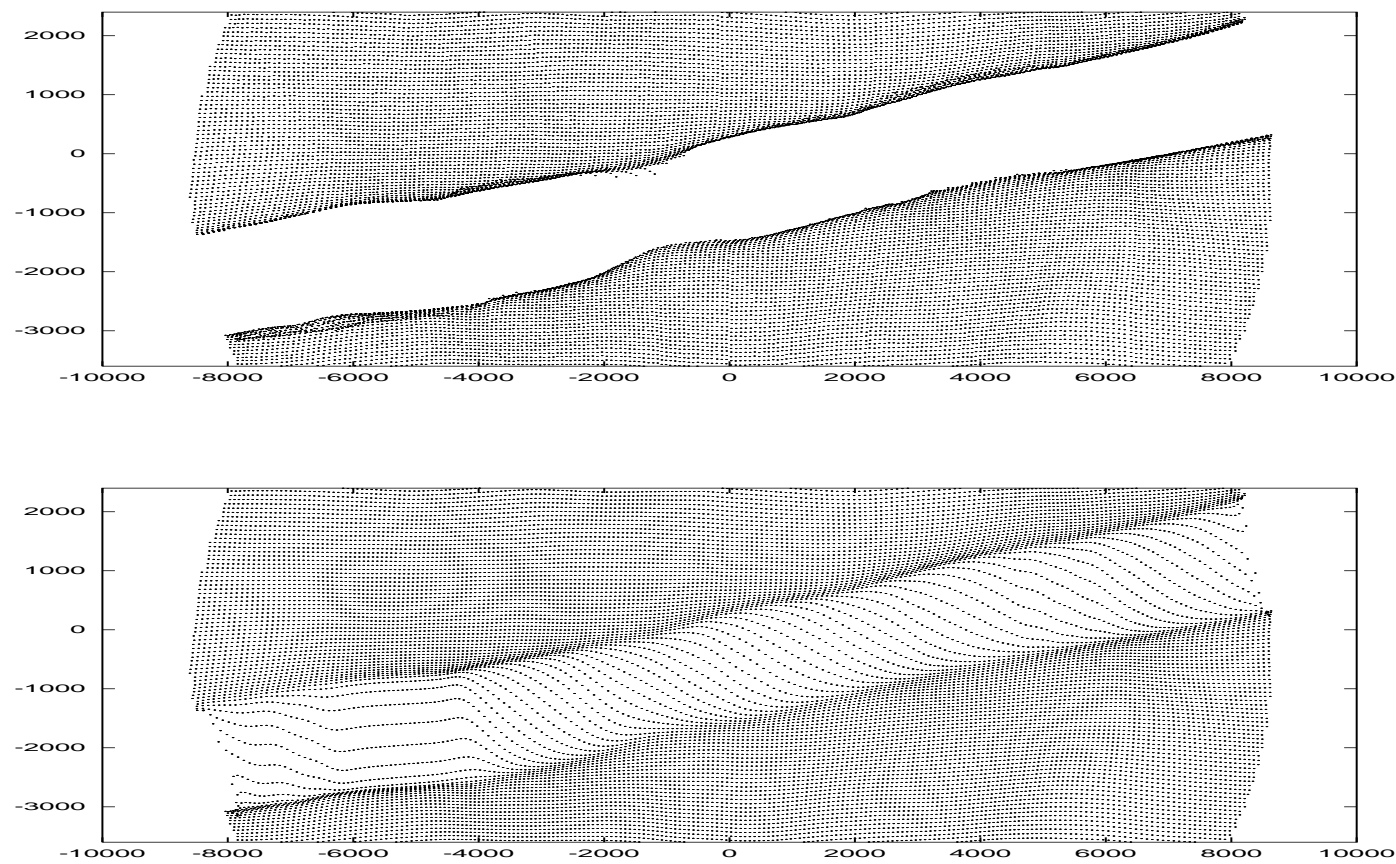

Abbildung 3.16: Oben: unrelaxiertes Pixelraster, unten: relaxiertes Pixelraster

Die nun folgenden Messungen und Korrektionen sind mit dem gebrochen Kristall angefertigt worden. Der Bruch des Kristalls zeigt deutlich die Grenzen des Verfahrens: Ist die Eindeutigkeit zwischen dem geometrischen Verlauf der Rasterpunkte $(i, j)$ und der zugehörigen Mittelwerte $\bar{x}_{\gamma}(i, j)$ und $\bar{y}_{\gamma}(i, j)$ nicht mehr gegeben, so ist keine Korrektion mehr möglich. In der Abbildung 3.16 sieht man beim Hochwandern längs einer Spalte, wie die Mittelwerte $\bar{x}_{\gamma}(i, j)$ und $\bar{y}_{\gamma}(i, j)$ in der Nähe des Bruchs ihre Richtung umkehren, die 
Spalte also wieder abwärts wandern - obwohl die Rasterpunkte $(i, j)$ der Feinnadelstrahlquelle weiter in der Spalte aufwärts wandern. Um jedoch die Auswirkungen dieses Bruchs so klein wie möglich zu gestalten, mußte das Pixelraster nachbearbeitet werden. Grundlage dieser Nachbearbeitung bildet die Kenntnis der Nachbarschaftsbeziehungen zwischen einzelnen Rasterpunkten $(i, j)$. Nun ist es möglich, zwischen einzelnen Nachbarn „Federn zu spannen“, eine resultierende Kraft zu berechnen und die Koordinaten des Mittelwertes $\bar{x}_{\gamma}(i, j)$ und $\bar{y}_{\gamma}(i, j)$ in Richtung dieser Kraft zu verschieben. Bei der Berechnung der resultierenden Kraft gehen nur diejenigen Federn ein, die jeweils zu den nächsten Nachbarn gespannt sind: Die Kraft (als zweidimensionaler Vektor im Koordinatensystem der Gammakamera) $\vec{F}(i, j)$ auf den Rasterpunkt $(i, j)$ mit den Koordinaten $\vec{x}_{\gamma}(i, j)=\left(\begin{array}{l}x_{\gamma} \\ y_{\gamma}\end{array}\right)$ ergibt sich somit zu

$$
\vec{F}(i, j)=\sum_{\substack{k=-1 \\ l=-1}}^{1} K \cdot\left(\vec{x}_{\gamma}(i, j)-\vec{x}_{\gamma}(i+k, j+l)\right)
$$

$K$ stellt die „Federkonstante“ dar. Diese Form der Kraft ( (3.8) stellt gewissermaßen eine lokale Wechselwirkung dar, die die größeren Zusammenhänge unbeeinflußt läßt. Nach Wahl einer geeigneten Federkonstante $K$ kann iterativ das Pixelraster lokal relaxiert werden. Während eines jeden Iterationsschritts wird dabei ein Rasterpunkt in Richtung der resultierenden Kraft verschoben um einen Betrag, der zum Betrag der resultierenden Kraft proportional ist:

$$
\delta \vec{x}_{\gamma}(i, j)=\alpha \cdot \vec{F}(i, j)
$$

Die hier auftretende Proportionalitätskonstante $\alpha$ wird exponentiell von Iteration zu Iteration gedämpft in grober Anlehnung an das als „simulierte Abkühlung“ (simulated annealing) bezeichnete Verfahren [19]. Die randständigen Rasterpunkte werden bei diesem Verfahren als fest betrachtet und nicht verschoben.

In Abbildung 3.16 ist das Ergebnis für das Raster um den Spalt herum aufgetragen. Es ist zu erkennen, daß das Raster seine Gestalt grundsätzlich behält. Um die Spaltkanten herum wird es jedoch in der Form auseinandergezogen, so daß die geometrische Eindeutigkeit bis nahe an den Rand des Spaltes aufrechterhalten wird. Im nächsten Abschnitt 3.2 kann in der Abbildung 3.26 die unterschiedliche Wirkung auf ein Szintigramm betrachtet werden, welches einmal mit Hilfe des unbehandelten und einmal mit Hilfe des nachbehandelten Punkterasters erstellt wurde. Als Nebeneffekt des Relaxierens ergibt sich der Ausgleich von kleinen Fehlern in der Positionierung der Feinnadelstrahlquelle, wie überhaupt einer höheren Toleranz stochastischen Schwankungen jeglicher Ursache gegenüber. 

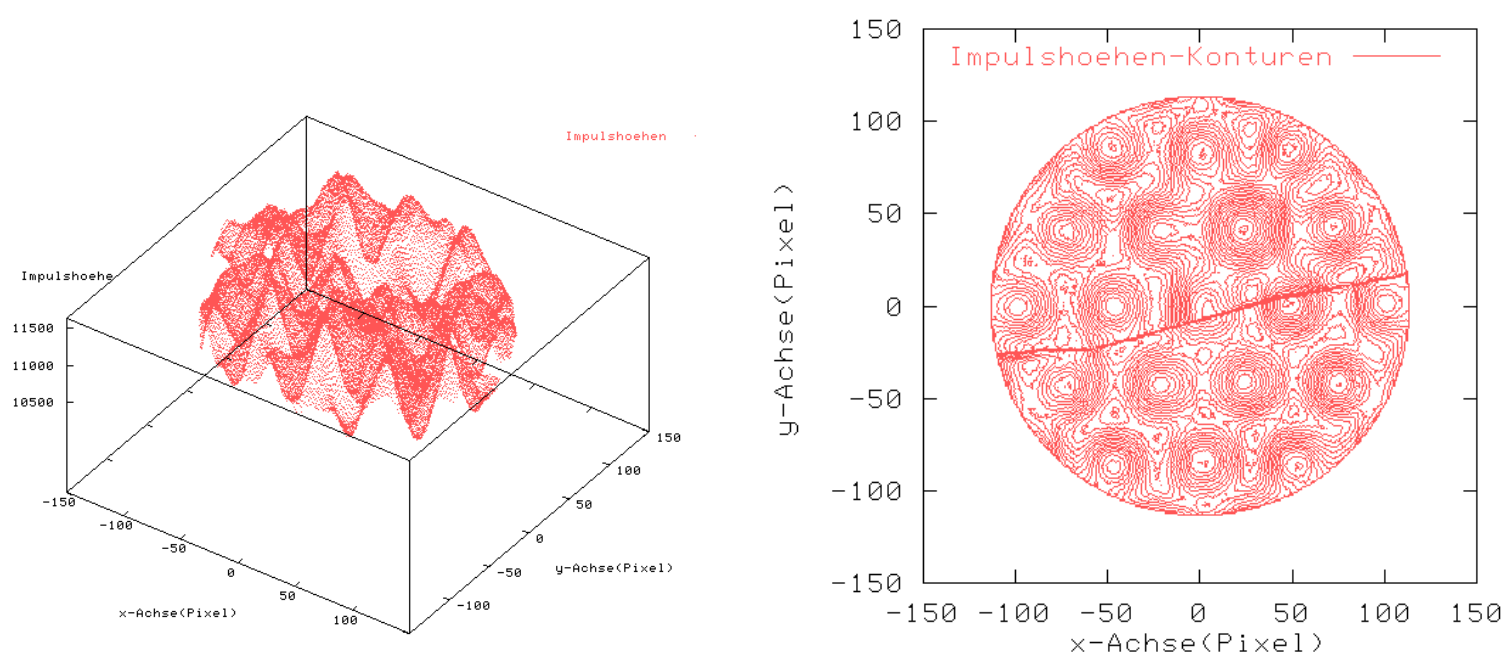

Abbildung 3.17: Die Impulshöhe als Funktion des Rasterindex $(i, j)$. Der linke Teil der Abbildung zeigt die Berg- und Tallandschaft der mittleren Impulshöhe an dem Rasterpunkt. Der rechte Teil zeigt den gleichen Sachverhalt, allerdings als Konturplot.

Die Mittelwerte der Impulshöhenantwort $\bar{E}_{\gamma}(i, j)$ lassen sich als Funktion des Rasterindex $(i, j)$ darstellen. Auf diese Weise ist die Darstellung keinen geometrischen Verzerrungen unterworfen, die sich ergeben würden, wenn $\bar{E}_{\gamma}(i, j)$ über $\bar{x}_{\gamma}(i, j)$ und $\bar{y}_{\gamma}(i, j)$ aufgetragen würde. ergibt sich eine Berg- und Tallandschaft, hervorgerufen durch die Anordnung und Eigenschaften der Photomultiplier, wie sie in der Abbildung 3.17 dargestellt ist. Auch hier ist der Bruch des Detektionskristalls deutlich zu erkennen. Die beobachtbaren Schwankungen betragen - ohne Berücksichtigung des Bereichs um den Bruch herum - \pm 5 der über den gesamten Detektor gebildeten mittleren Impulshöhe. Im Bereich um den Bruch bzw. um den absichtlich dekalibrierten Photomultiplier Nr.33 herum (im linken oberen Viertel der rechten Abbildung 3.17) können die Abweichungen noch weitaus größer sein.

Bemerkenswert ist die Beobachtung, daß die Maxima der Impulshöhen nicht zentral zu den Photomultipliern liegen, sondern in den Zwischenräumen lokalisiert sind. Dies hat die Ursache in sogenannten Masken, die auf den Lichtleiter aufgebracht sind und die Nichtlinearität bzgl. der Impulshöhenantwort verringen sollen. Leider war vom Hersteller nichts über die genaue Form dieser Maske zu erfahren.

Aber auch die Schwankung der mittleren Impulshöhe von Rasterpunkt zu Rasterpunkt können erheblich sein, wie die Abbildung 3.18 zeigt. In dieser Abbildung sind zwei willkürlich ausgewählte Schnitte durch die Impulshöhenfläche der Abbildung 3.17 bei $x=0$ und $x=10$ dargestellt.

Aufgrund der hohen Empfindlichkeit der Bildgüte bzgl. der Impulshöhe ist eine örtlich 
Abbildung 3.18:

Zwei Schnitte bei $x=0$ und $x=$ 10 durch die Fläche der mittleren Impulshöhe pro Rasterpunkt der Abb. 3.17.

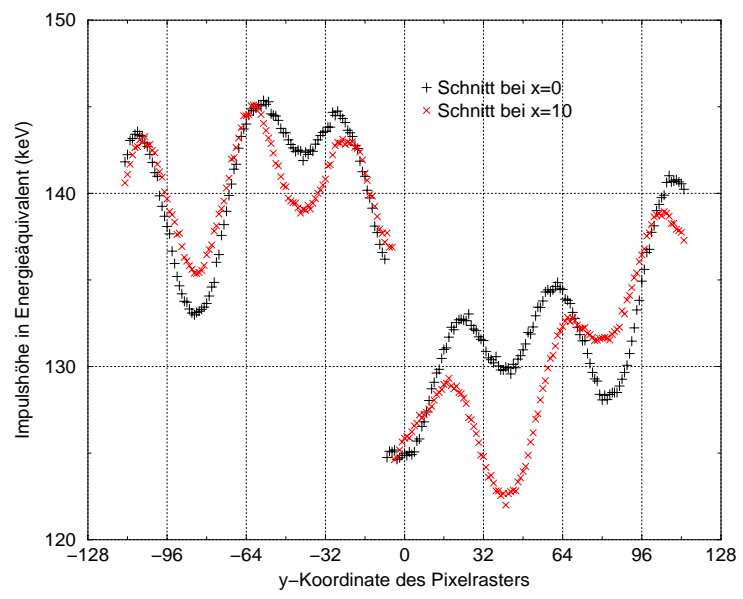

hoch aufgelöste Impulshöhenbetrachtung so wichtig. Hier liegt einer der großen Vorteile des Feinnadelstrahlquellenverfahrens gegenüber der sonst üblichen Korrektion der Impulshöhe aufgrund von flächenhaften Aktivitätsverteilungen.

\subsection{Das Verfahren in der Anwendung}

In diesem Kapitel werden Beispiele für die Anwendung des im vorhergehenden Kapitel beschriebenen Verfahrens vorgestellt. Alle hier gezeigten Bilder korrespondieren mit sog. List-Modus-Messungen, d.h. jedes einzelne detektierte $\gamma$-Quant wurde mit den beiden unkorrigierten Ortskoordinaten, der zugehörigen Impulshöhe und dem Zeitpunkt der Detektion registriert und in ein File auf die Festplatte eines angeschlossenen Computers geschrieben. Der Vorteil dieses Verfahrens ist, daß auch nach Abschluß der Aufnahme ohne Einschränkung alle Informationen wie bei der Aufnahme selbst zur Verfügung stehen. Auch im Nachhinein können so z.B. beliebige Impulshöhenfenster gelegt werden.

Abbildung 3.19:

Korrektion der simulierten Abbildung einer Gammakamera.

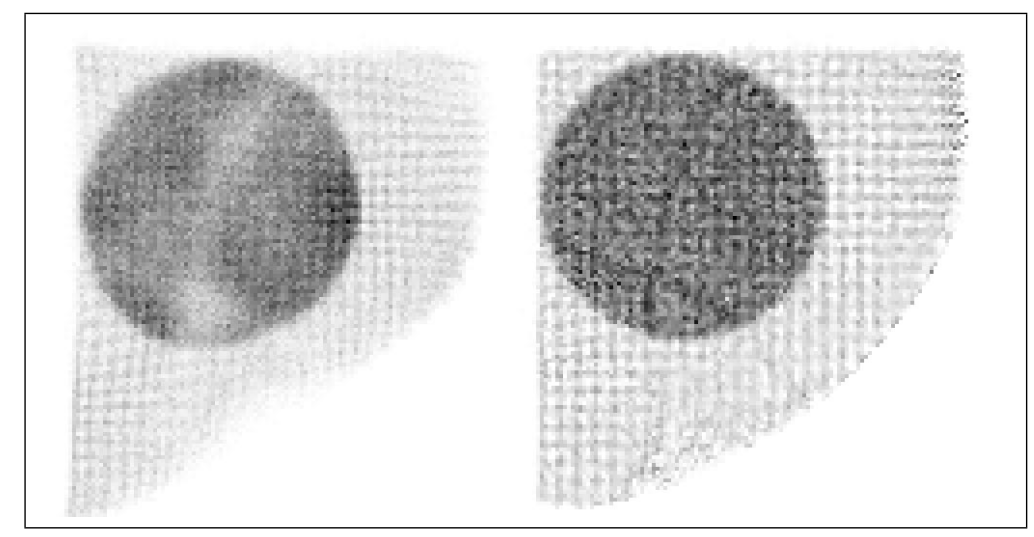


Mit Hilfe der im Abschnitt 2.4 vorgestellte Simulation konnte vorab getestet werden, ob das Verfahren als solches funktioniert. Dazu wurde der fiktiven Kamera eine kreisförmige homogene Verteilung überlagert von einem aus Streifen bestehenden Gitter angeboten. Das Ergebnis ist im linken Teil der Abbildung zu sehen. Ebenso mußten die Messungen der einzelnen Rasterpunkte simuliert werden, deren Ergebnisse dann zu der Korrektion benutzt wurden, deren Ergebnis im rechten Teil zu sehen ist. Das Ergebnis gab Anlaß, das Verfahren auch in die Realität umzusetzen.

Wie schon im vorangegangenen Kapitel erwähnt, ist der Detektionskristall der Gammakamera während der Anfertigung dieser Arbeit gebrochen. In den Abbildungen ist dies als „Spalt“ zu erkennen, in dem keinerlei Quanten registriert zur werden scheinen. Daneben ist der Photomultiplier mit der Nr.33 (siehe Abbildung 3.8) absichtlich dekalibriert, und zwar in der Weise, daß er ein zu schwaches Signal erzeugt. Das vorgelegte Verfahren ist in der Lage die Konsequenzen aus dieser Dejustage für das Abbildungsverhalten der Kamera vollständig aufheben, da die Eineindeutigkeit der Abbildung erhalten bleibt. Dies war bei den Auswirkungen des Kristallbruchs nicht der Fall.

Abbildung 3.20:

Unkorrigierte Abbildung einer fernen Punktquelle.

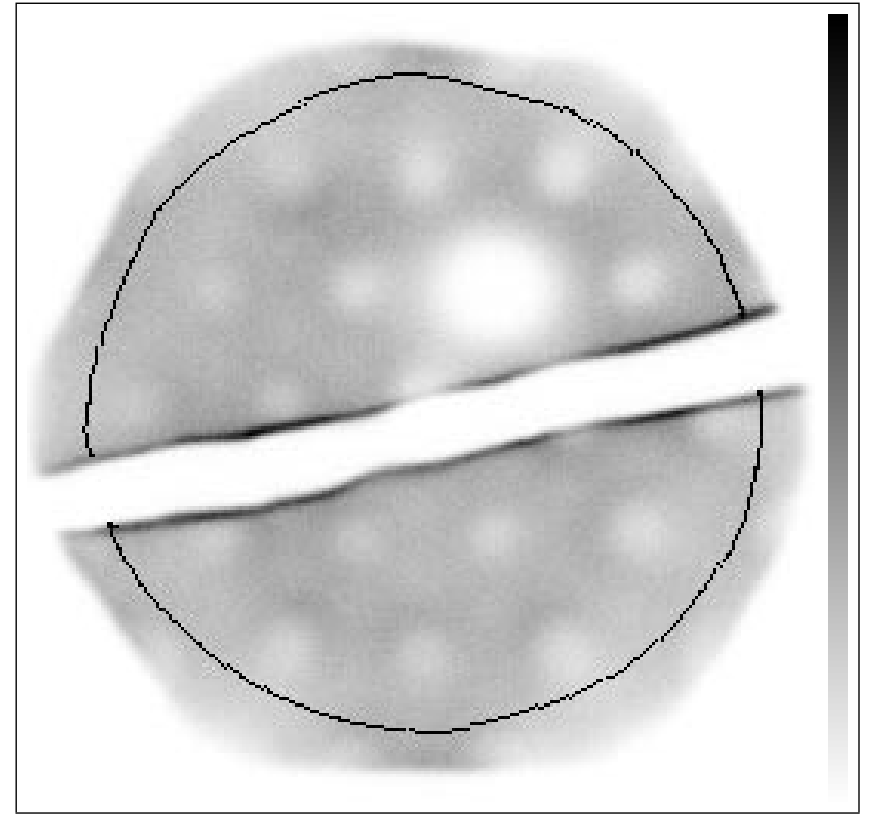

In der Abbildung 3.20 ist das Ergebnis einer Aufnahme ohne Kollimator einer fernen Punktquelle gezeigt, wobei ein Impulshöhenfenster von 10\% um den der Energie von $140 \mathrm{keV}$ entsprechenden Kanal gelegt ist. Die Bildmatrixgröße entspricht 256x256 Pixel und die Pixelkantenlänge $1.1 \mathrm{~mm}$ durch das Verfahren in $1.1 \mathrm{~mm}$ Schritten. Zusätzlich ist 
als schwarze Linie der Rand desjenigen Bereichs eingezeichnet, der von den Rasterpunkten überdeckt wird. Der Bereich außerhalb dieser Grenze ist mit diesem Verfahren keiner Korrektur zugänglich, allerdings liegt dieser auch außerhalb des als benutzbar angesehenen Bereichs. Die Anzahl der $\gamma$-Quanten innerhalb dieses Bereichs und innerhalb des beschriebenen Impulshöhenfensters ist bei dieser Aufnahme 13158782.

Bei der Aufnahme betrug die Entfernung der Punktquelle zum Detektor etwa $3 \mathrm{~m}$, so daß eine homogene Verteilung der Quanten über den Detektor gegeben ist. Insgesamt sind während der Aufnahme 30 Millionen Quanten über alle Impulshöhen und den gesamten Kristallbereich registriert worden.

Das Abbildung 3.20 zeigt deutlich die zu erwartenden Inhomogenitäten. Diese sind um den Photomultiplier mit der Nr.33 besonders ausgeprägt, da er ein zu schwaches Signal liefert und in Folge die Impulshöhen der hier detektierten $\gamma$-Quanten im Mittel unterhalb des angelegten Impulshöhenfensters liegen (siehe dazu auch Bild 3.17, welches die Impulshöhen aufgetragen über die Detektorfläche zu dieser Aufnahme darstellen.). Die Schwärzung entlang beider Spaltgrenzen erklärt sich durch das Zusammenlaufen derjenigen Quanten, die im Bereich um den Spalt herum auftreffen.

Abbildung 3.21:

Korrigierte Abbildung einer fernen Punktquelle.

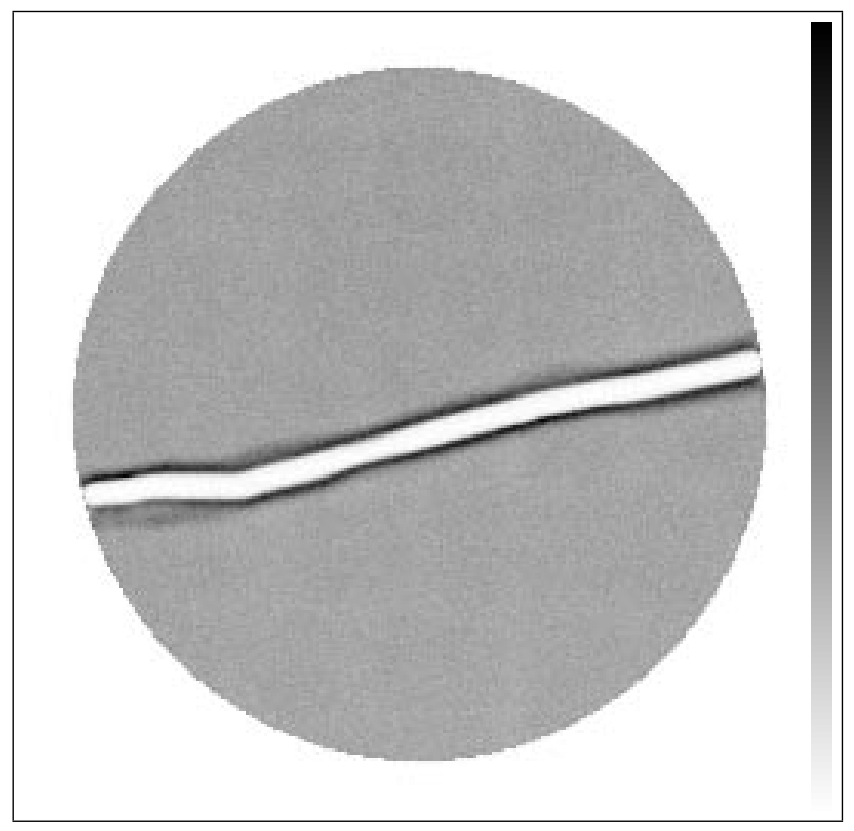

Die korrigierte Aufnahme der fernen Punktquelle ist in der Abbildung 3.21 dargestellt. Von den 30 Millionen Quanten können 6485902(21,62\%) nicht in das Raster einsortiert werden, liegen also außerhalb des mit der schwarzen Linie gekennzeichneten Bereichs. Von 
den verbliebenen 23514100 Quanten liegen 13845516 innerhalb des Impulshöhenfensters und werden bildwirksam.

Aus beiden Szintigrammen ist als Profillinie der Schnitt durch die Mitte bei $x=0$ in der Abbildung 3.22 dargestellt. Die Schnittführung und -richtung sind durch den blauen bzw. schwarzen Pfeil gekennzeichnet. Im Falle des korrigierten Bildes liegt der Mittelwert der Belegung bei 345 und um diesen herum ist das $3 \sigma$-Intervall als farbiger Hintergrund angedeutet. Bis auf zwei Ausnahmen liegen alle Werte innerhalb dieses Intervalls; dies mag als Hinweis darauf gesehen werden, daß die verbleibenden Schwankungen allein auf die Poissonverteilung zurückzuführen sind.

Abbildung 3.22:

Schnitt entlang einer Geraden $x=0$.

Die Pfeilrichtung gibt die Richtung der Schnittführung an. Im unkorrigierten Bild ist der Einfluß des verstellten Photomultipliers Nr.33 deutlich zu erkennen.

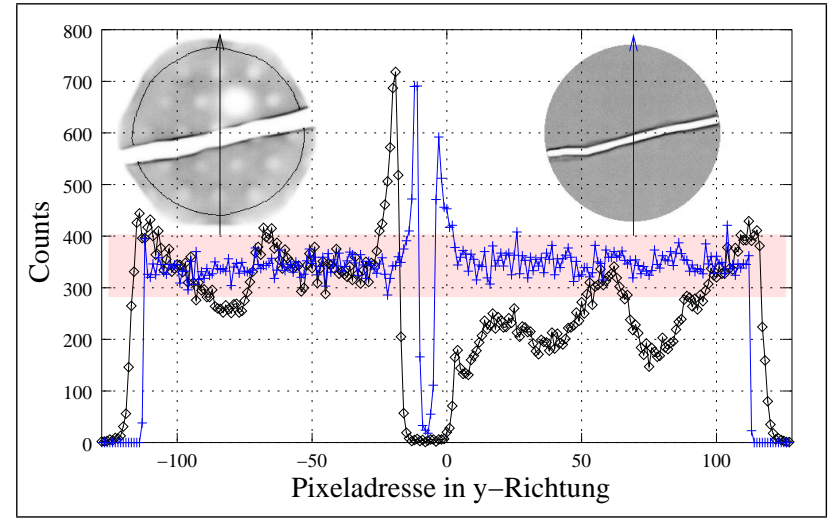

Besonders schön ist die Zusammenschnürung des Spaltes auf knapp ein Drittel seines unkorrigierten Ausmaßes. Dies ist nur mit dem hier vorgestellten Verfahren möglich, herkömmliche Verfahren mit einer Rasterbildung vor dem Korrektionschritt würden viel von der hier noch möglichen eindeutigen Zuordnung zwischen Detektionsort und wahrscheinlichst als wahr anzunehmenden Ort aufgeben mit der Folge eines breiteren Spaltes. In der Abbildung 3.23 ist die Impulshöhenverteilung für die Aufnahme der fernen Punktquelle aufgetragen und zwar einmal vor (blaue Linie) und einmal nach der Korrektion (rote Linie). Die Auftragung ist so normiert, daß die Fläche unterhalb der Kurven jeweils vom Betrag 1 ist. Neben der reinen Verschiebung des Photopeaks (Maximum, ungefähr bei $134 \mathrm{keV}$ ) ist deutlich zu erkennen, daß die Breite des unkorrigierten Photopeaks größer ist als die der Korrigierten. Betrachtet man z.B. die volle Breite der Verteilung bei halber Höhe des Maximums (FWHM) - in der Abbildung 3.23 als schwarze Pfeile aufgetragen , so ist sie im unkorrigierten Fall $22 \mathrm{keV}$, im korrigierten dagegen nur $15 \mathrm{keV}$. Grau hinterlegt ist das jeweils zur Erstellung der Szintigramme angelegte Impulshöhenfenster von $\pm 10 \%$, welches demnach von $126-154 \mathrm{keV}$ reicht. Die erreichte hohe Güte der Korrektion 
Abbildung 3.23:

Impulshöhenverteilung der Aufnahme einer entfernten Punktquelle, vor und nach der Korrektion. Die Flächen unterhalb der Kurven sind normiert, die Breite eines Kanals beträgt $1 \mathrm{keV}$. Die beiden Pfeile kennzeichnen die Breite der jeweiligen Verteilung bei halber Höhe des Maximums.

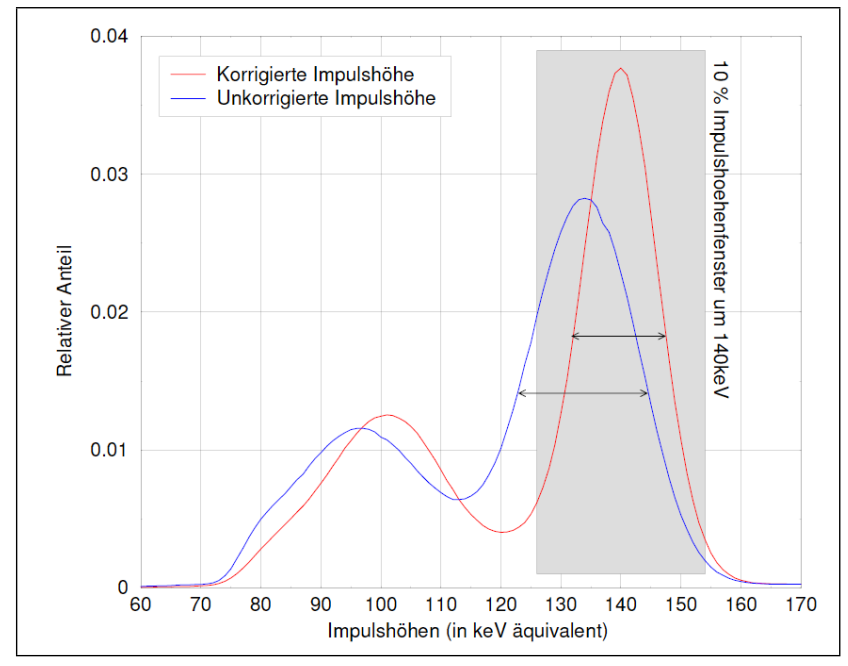

läßt sich auch an der Lage der Flanken des Photopeaks ablesen, beide treten fast auf gleicher Höhe aus dem grau unterlegten Bereich hinaus (Symmetrie).

Um neben der Wirkung der Korrektionvorschrift auf die Homogenität der Abbildung die Leistungsfähigkeit in Hinblick auf die Linearität zu untersuchen, müssen der Kamera Gammakamera Quanten angeboten werden, die in Form gleichmäßiger geometrischer Muster auf den Detektorkristall treffen. Dabei stehen neben den beiden üblichen Methoden, die Muster entweder durch Abschirmung bestimmter Areale des Detektors oder durch Einbringung aktiver Lösung in geeignet geformte Volumina ${ }^{7}$, eine weitere, mit Hilfe der Feinnadelstrahlquelle realisierbare Möglichkeit zur Verfügung: durch die freie Verfahrbarkeit der Feinnadelstrahlquelle kann nahezu jedes beliebe zweidimensionale Muster erzeugt werden.

In dieser Arbeit sind alle drei im vorhergehenden Absatz erwähnten Methoden eingesetzt. Zunächst werden Muster erzeugt, indem in Plexiglas eingelassene Blei- bzw Alumuster auf den Detektorkristall gebracht werden. Für einen homogenen Fluß von $\gamma$-Quanten und sorgt wie im Falle der Homogenitätsaufnahme eine ferne Punktquelle. Die Bleimuster sind von hinreichender Stärke, um für eine totale Abschirmung zu sorgen, so daß ein Negativ des Bleimusters als Szintigramm entsteht. In der Abbildung 3.24 ist ein derartiges Szintigramm eines Musters aus einer Bleiplatte mit äquidistanten Bohrungen gezeigt. Jede dieser Bohrungen besitzt einen Durchmesser von $2.8 \mathrm{~mm}$ und die Abstände der Bohrungen voneinander beträgt $12.7 \mathrm{~mm}$.

\footnotetext{
${ }^{7}$ In diesem Fall muß die Abbildung allerdings mit einem Kollimator gemacht werden.
} 
Abbildung 3.24:

Unkorrigierte Abbildung einer fernen Punktquelle. Der Detektorkristall wird mit einer Aluminiumplatte abgedeckt, die Bohrungen mit dem Durchmesser von $2.8 \mathrm{~mm}$ besitzt. Die Bohrungen haben den Abstand von $12.7 \mathrm{~mm}$.
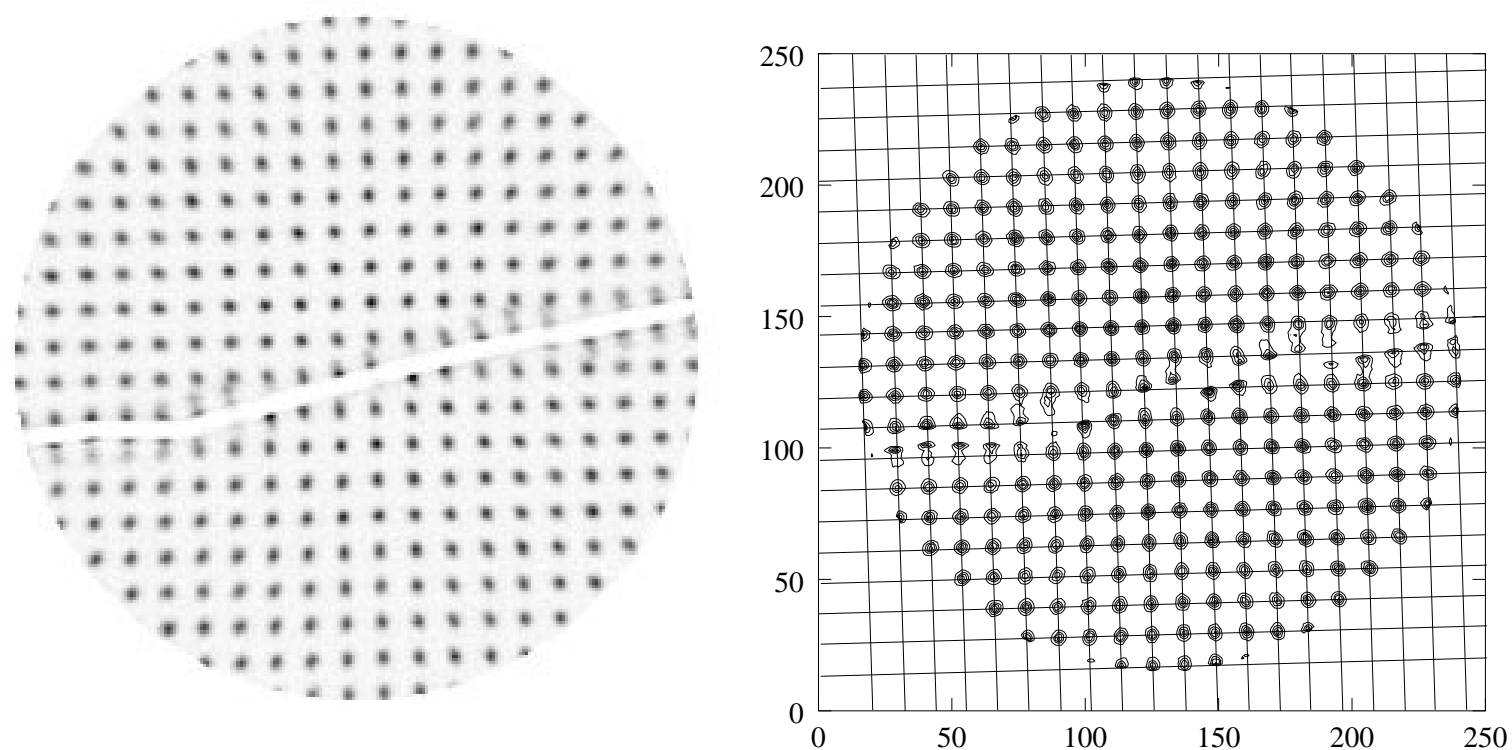

Abbildung 3.25: Korrigierte Aufnahme, links Szintigramm, rechts Konturauftragung des Szintigramms. 
Deutlich sind in der Abbildung 3.24 die Verzerrungen an der Lage der Punkte zueinander ablesbar, wie z.B. die erwartete tonnenförmige Verzerrung um den absichtlich verstellten (in Richtung auf ein zu kleines Signal) Photomultiplier Nr.33 zu erkennen.

Das Ergebnis der Korrektion des Szintigramms ist in dem linken Teil der Abbildung 3.25 gezeigt. Von den drei Millionen Quanten, die für dieses Szintigramm aufgenommen wurden sind nach Korrektion 1.401.742 bildwirksam, wobei 669.414 Quanten außerhalb des korrigierbaren Bereichs liegen.

Die hohe erreichte Linearität der Abbildung soll mit dem rechten Teil der Abbildung 3.25 dargelegt werden. Hier sind die Konturlinien des Szintigramms aufgetragen, wobei jeder Konturlinie 50 Quanten entspricht. Die Einheiten auf der Abszisse und Ordinate entsprechen einem Pixel. Wie zu erkennen, verlaufen die Zentren der Verteilungen ,die zu den einzelnen Punkten gehören, durch die Schnittpunkte eines regelmäßigen Linienmusters, welches über den Konturplot gelegt ist. Die Abstände der Linien voneinander beträgt $12.8 \pm 0.2 \mathrm{~mm}$ oder 11.63 Pixel in guter Übereinstimmung mit den Abständen auf dem Muster selbst.

Im vohergehenden Abschnitt 3.1.4 ist die Nachbearbeitung des der Korrektion zugrunde liegenden Pixelrasters dargelegt worden. Die Wirkung dieser Nachbearbeitung soll anhand eines Bildausschnitts des Punktemusters um den Bruch des Kristalls verdeutlicht werden. In der Abbildung 3.26 sind zwei Korrektionen gegenübergestellt. Im linken Teil der Ab-
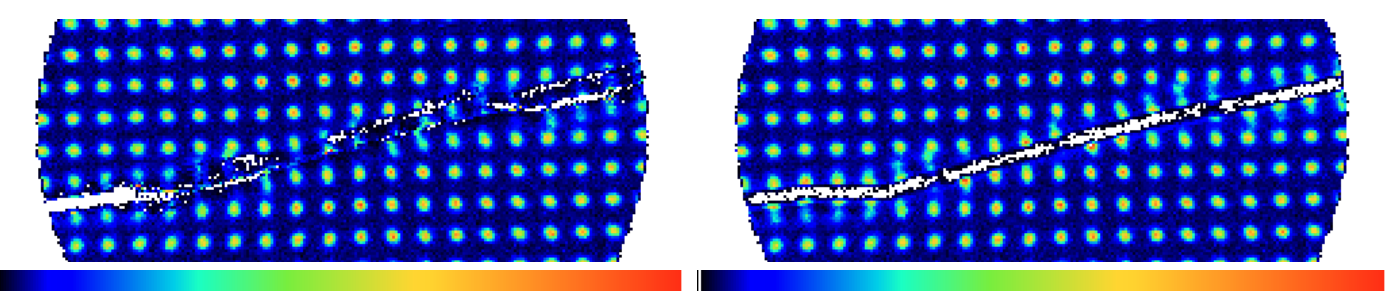

Abbildung 3.26: Vergleich zwischen Wirkung von unrelaxiertem und relaxierten Pixelraster.

bildung findet sich das Ergebnis der Korrektion mit Hilfe eines Satzes nicht nachbearbeiteter Rasterpunkte, im rechten Teil dagegen ist ein nach dem in Abschnitt dargelegten Verfahren „relaxiertes“ Gitter aus Rasterpunkten die Grundlage der Korrektion, die hier dargestellt ist. Unterscheiden sich die beiden Ergebnisse in hinreichendem Abstand vom Bruch kaum, so erreicht man mit dem nachbearbeiteten Datensatz um den Bruch herum eine weitreichendere Aufrechterhaltung der Eineindeutigkeit in der Zuordnung von Detektionsort zu Rasterpunkt.

Neben der Platte mit regelmäßigen Bohrungen standen noch zwei weitere Muster zur Verfügung. Die Abbildungen 3.27 und 3.28 zeigen jeweils auf der linken Seite die unkorri- 
gierte, auf der rechten Seite die korrigierte Version des Szintigramms zu diesen Mustern. Hierbei ist zu beachten, daß zur Bildung der unkorrigierten Version keinerlei Impulshöhenfenster genutzt wurde, um die Vergleichbarkeit zur korrigierten Fassung in Bezug auf die Linearität zu steigern. Zur Bildung der korrigierten Fassung ist dagegen ein Impulshöhenfenster von 126-154 keV zur Anwendung gelangt. Die Fläche innerhalb der schwarzen Linie stellt den Teil dar, der der Korrektion zugänglich ist. In beiden Fällen ist die durch die

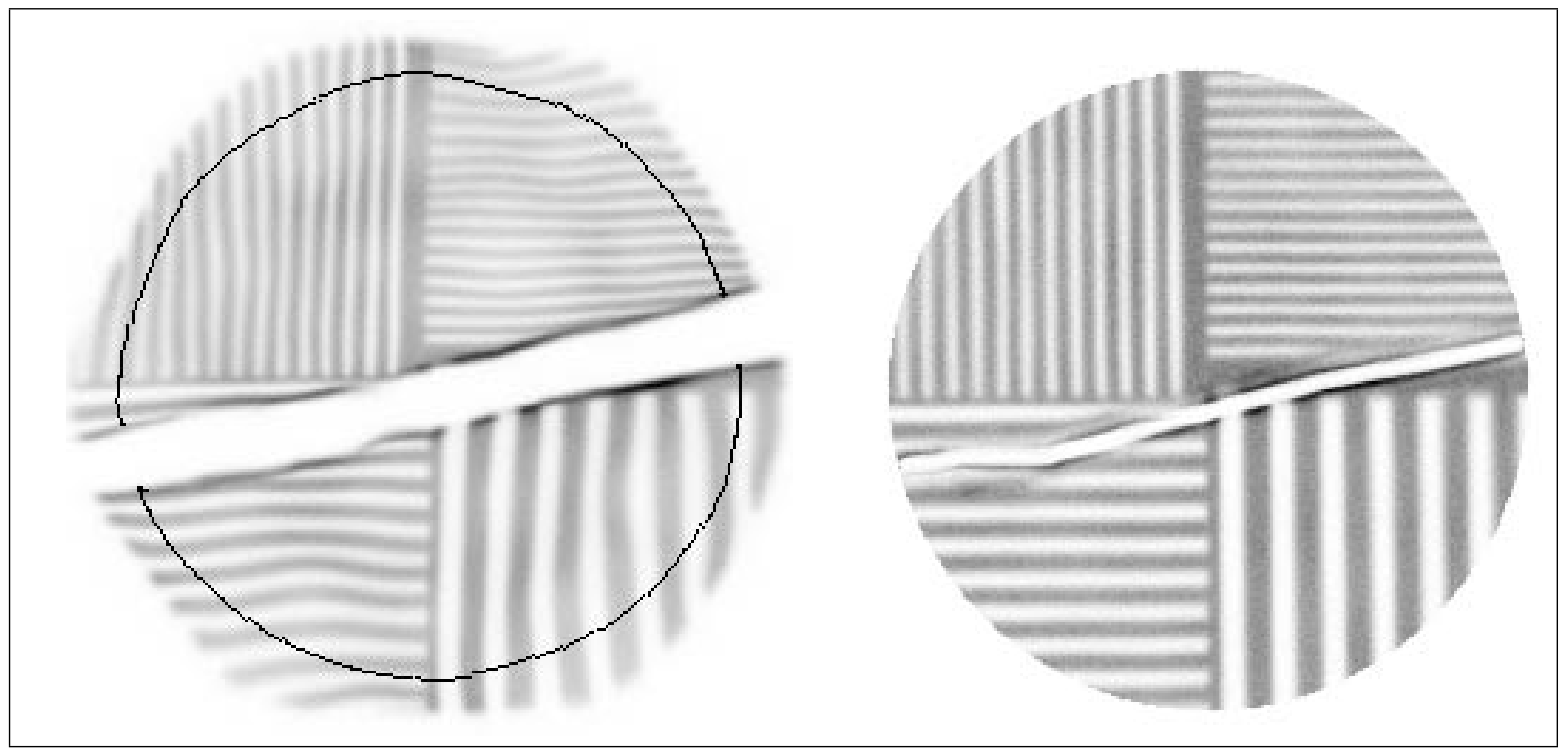

Abbildung 3.27: Muster aus Streifen verschiedener Stärke, linkes Teilbild unkorrigiert, rechtes Teilgebiet korrigiert. Die Stärken der Streifen betragen in den jeweiligen Quadranten: Rechts unten $0.95 \mathrm{~cm}(3 / 8 \mathrm{Zoll})$, links unten $0.64 \mathrm{~cm}(1 / 4 \mathrm{Zoll})$, links oben $0.48 \mathrm{~cm}$ (1/4 Zoll) und rechts oben $0.40 \mathrm{~cm}(5 / 32$ Zoll).

Korrektion erreichte Linearität sehr befriedigend. Wie oben erwähnt bietet die Feinnadelstrahlquelle nicht nur das Werkzeug zur Rasterpunktbildung, sondern sie kann auch zur Erzeugung von zweidimensionalen Mustern eingesetzt werden, von denen zwei in den Abbildungen 3.29 und 3.30 dargestellt sind, wobei die Abbildung 3.30 nicht eines der üblichen geradlinigen Muster zeigt, sondern kreisförmige Streifen.

Um die hohe erreichte Linearität zu unterstreichen, sind in beiden Bildern farbige Linien eingetragen, die entweder zu einer bestimmten, von der Feinnadelstrahlquelle abgefahrenen Bahn gehören (rote Linien) oder schlicht zur optischen Hervorhebung (blaue Linien dienen.

Im Unterschied zu den Abbildungen 3.27 und 3.28 ist hier auch bei der Erstellung der unkorrigierten Szintigramme das gleiche Impulshöhenfenster angewendet worden wie 


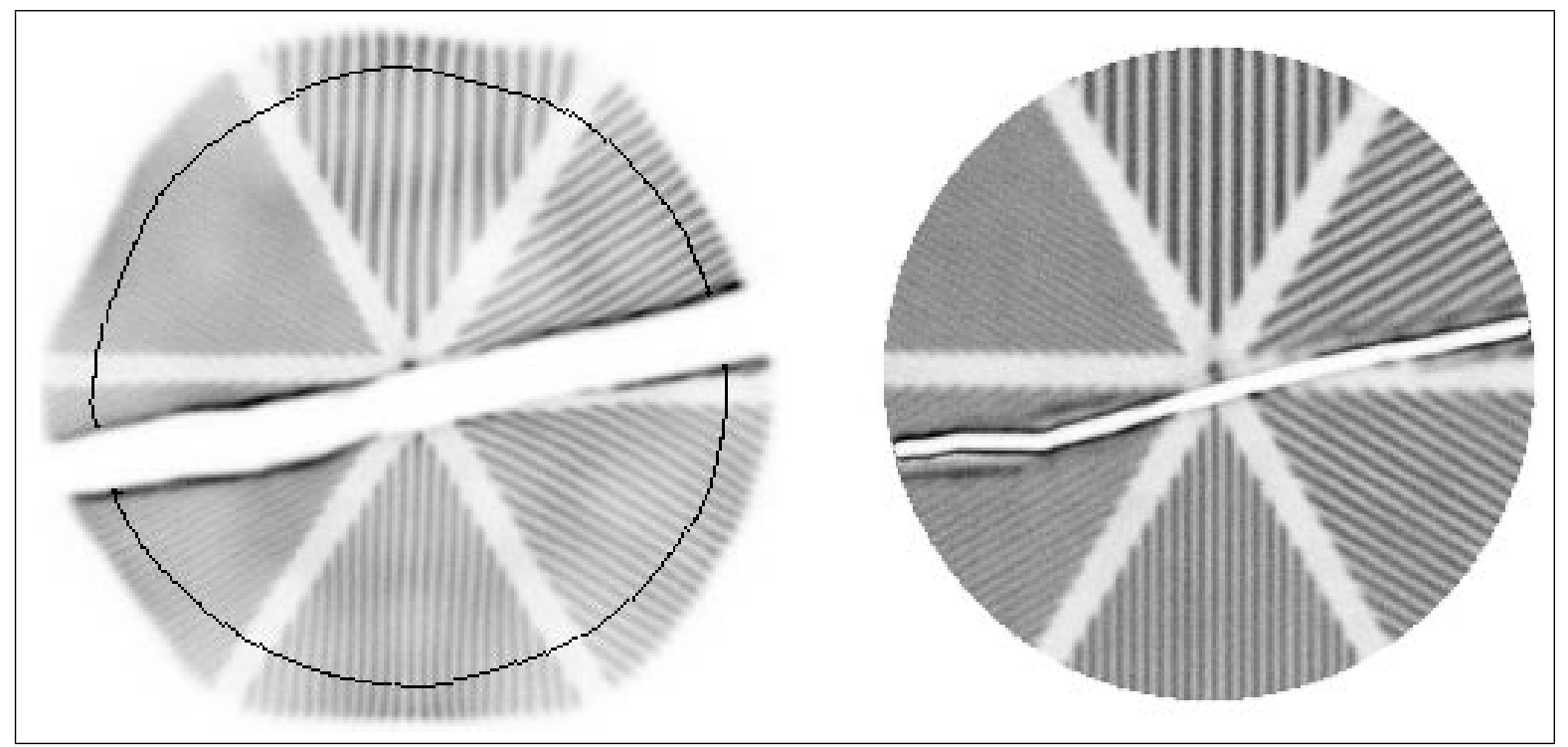

Abbildung 3.28: Muster aus Streifen verschiedener Stärke, linkes Teilbild unkorrigiert, rechtes Teilbild korrigiert. Die Stärke der Streifen beträgt in abfallender Folge (oberstes Sechstel beginnend, dann im Uhrzeigersinn umlaufend) $4 \mathrm{~mm}, 3.18 \mathrm{~mm} 2.77 \mathrm{~mm}, 2.54 \mathrm{~mm}$, $2.11 \mathrm{~mm}$ und schließlich $1.78 \mathrm{~mm}$.

bei den korrigierten. Alle bisherigen Szintigramme sind ohne Kollimator aufgenommen. Da die Vermessung der Gammakamera mit Hilfe der Feinnadelstrahlquelle ebenfalls ohne Kollimator erfolgt, kann die hier vorgestellte Korrektion etwaige Einflüsse des Kollimators nicht mit berücksichtigen. Die Trennung des Einflusses des Abbildungsverhaltens der Gammakamera ohne Kollimator einerseits und des Kollimators andererseits stellt ein sinnvolles Konzept dar, weil ein fehlerhafter Kollimator zu Szintigrammen führen kann, die schlicht falsch und somit keiner Korrektion sinnvoll zugänglich sind. Zeigt ein Szintigramm eines Testmusters welches mit einer durch die Feinnadelstrahlquelle vermessene Gammakamera erstellt ist, darüberhinaus Abweichungen, so sind diese auf den fehlerhaften Kollimator zurückzuführen. 


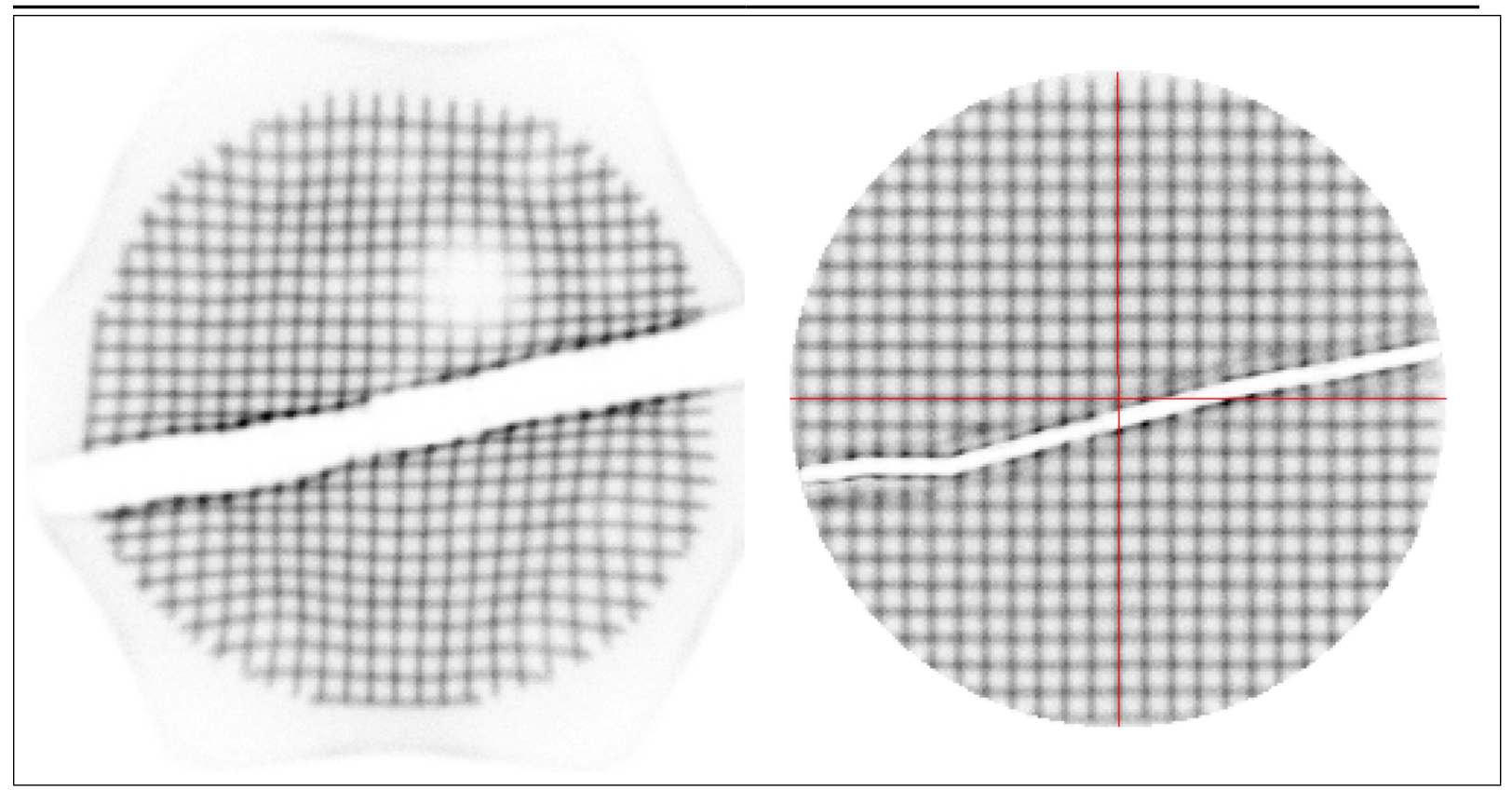

Abbildung 3.29: Durch Verfahren der Feinnadelstrahlquelle auf geraden Bahnen erzeugtes Muster, linkes Teilbild unkorrigiert, rechtes Teilbild korrigiert. Die Bahnen besitzen in beide Richtungen den Abstand $\Delta d=1 \mathrm{~cm}$ zueinander. Die roten Linien markieren geometrische Geraden, die senkrecht aufeinanderstehen und jeweils zur Bahn $y=0$ und $x=0$ gehören.

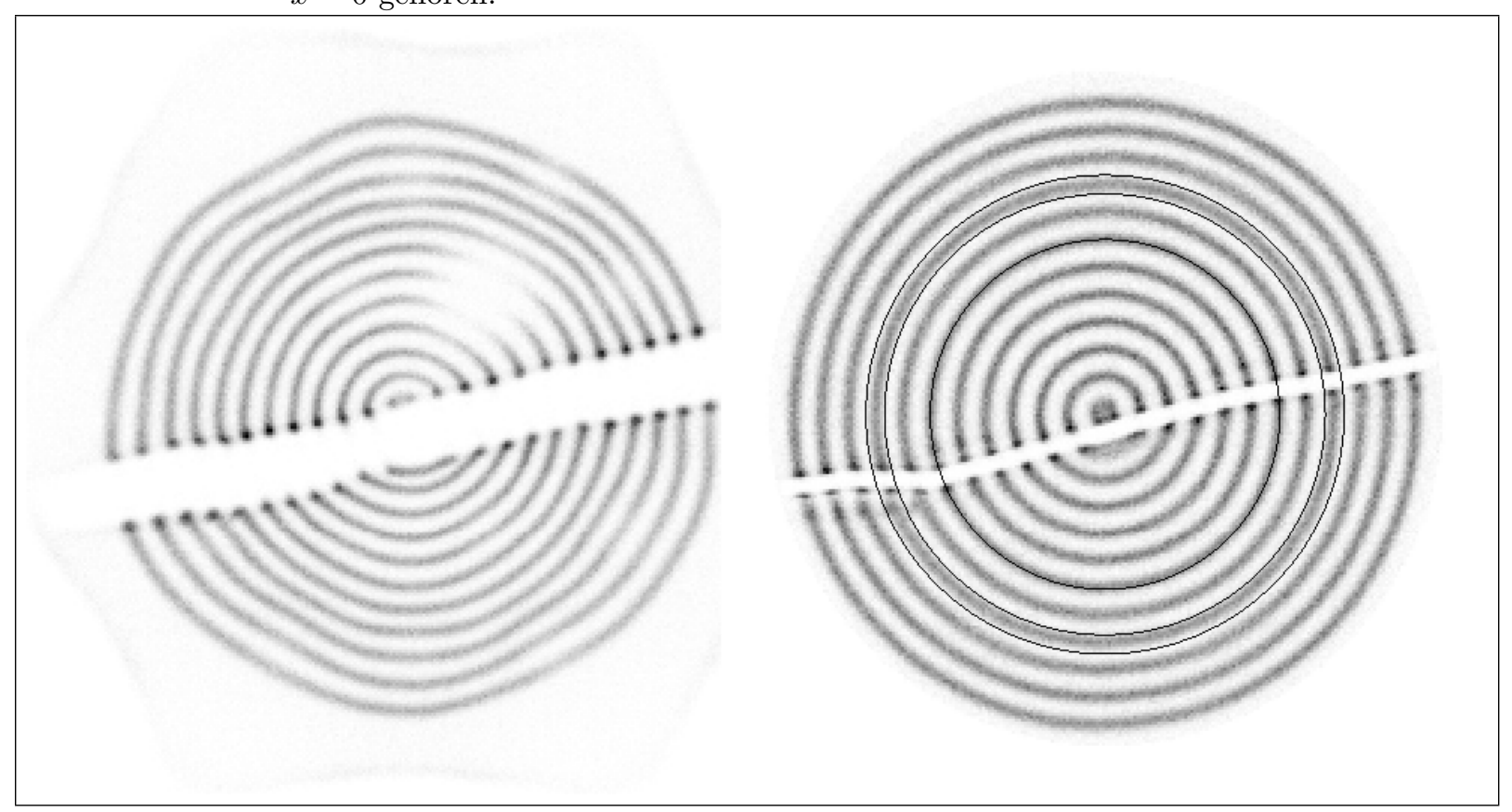

Abbildung 3.30: Durch Verfahren der Feinnadelstrahlquelle auf Kreisbahnen erzeugtes Muster, linkes Teilbild unkorrigiert, rechtes Teilbild korrigiert. Der Radius nimmt von Bahn zu Bahn um $\Delta r=1 \mathrm{~cm} \mathrm{zu}$, wobei der kleinste Kreis den Radius von $0.5 \mathrm{~cm}$ besitzt. Die rote Linie kennzeichnet einen geometrischen Kreis mit dem Radius von $6.5 \mathrm{~cm}$. Die blauen Kreise sollen die neunte Kreisbahn einrahmen. Sie besitzen die Radien $8.1 \mathrm{~cm}$ und $8.9 \mathrm{~cm}$. 
Um zu zeigen, daß das vorgestellte Verfahren auch bei der Verwendung eines Kollimators funktioniert, sind zwei in den Abbildungen 3.31 und 3.31 die Szintigramme eines Linienphantoms unter Verwendung zweier unterschiedlicher Kollimatoren gezeigt. Der eine Kollimator ist ein sogenannter Allzweckkollimator (general purpose) der andere ein speziell den Anforderungen der Schildrüsenszintigraphie angepaßter Kollimator, einfach Schilddrüsenkollimator genannt. ${ }^{8}$. Der Allzweckkollimator besitzt viele Bohrungen mit einem kleinen Durchmesser und kurzer Bohrungslänge, der Kollimator für die Szintigraphie der Schilddrüse dagegen wenige Bohrungen mit großem Durchmesser und langer Bohrungslänge.

Das Linienphantom besteht aus einem mit wässriger ${ }^{99 m}$ Tc -Lösung gefüllten Schlauch, der meanderförmig auf eine Plexiglasscheibe angebracht ist. Die parallelen Teile der Meanderstruktur verringern dabei den Abstand von Schleife zu Schleife um so das Auflösungsvermögen der Abbildung darzustellen. Der größte Abstand beträgt $2.54 \mathrm{~cm}$, der allerdings nur am rechten Rand des Szintigramms 3.31 sichtbar ist. Das Gesichtsfeld des Schilddrüsenkollimators ist zu klein, um das gesamte Phantom zu erfassen.

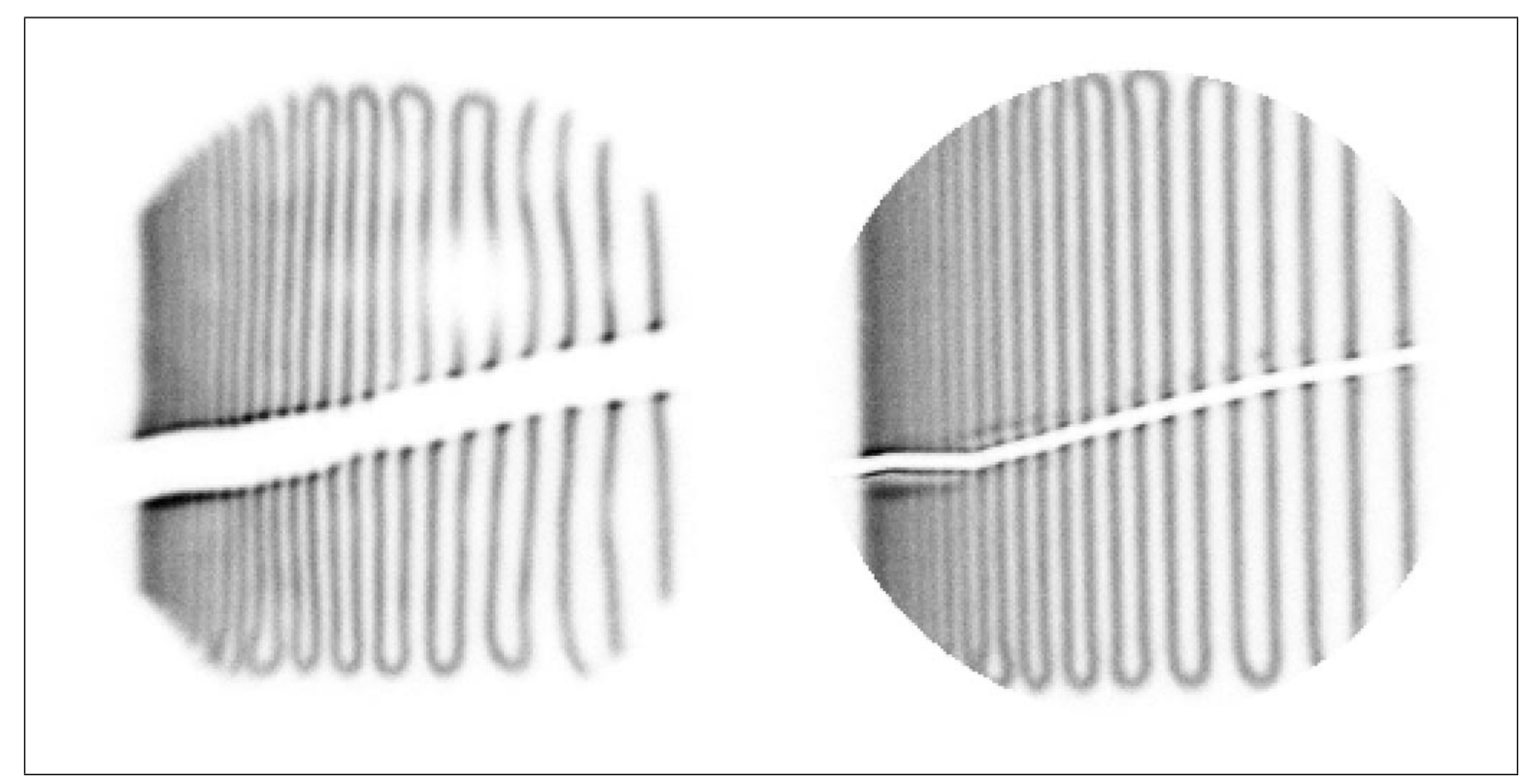

Abbildung 3.31: Szintigramm eines Linienphantoms, aufgenommen mit einem Kollimator für allgemeine Zwecke (general purpose).

\footnotetext{
${ }^{8}$ Die Picker CX-250 compact wird in der Nuklearmedizin der Universitätskliniken Göttingen meist zu Untersuchungen der Schilddrüse verwendet.
} 
Den Szintigrammen kann entnommen werden, daß das vorgestellte Verfahren ohne Probleme auch auf die kollimatorbewehrte Gammakamera angewendet werden kann. Die Streifenbildung im Szintigramm 3.32 ist eine Folge der relativ großen Septenstärke des Schildrüsenkollimators, die allerdings bei gröberer Rasterung als der hier verwendeten, wie sie für diesen Kollimator auch vorgesehen sind, verschwindet.

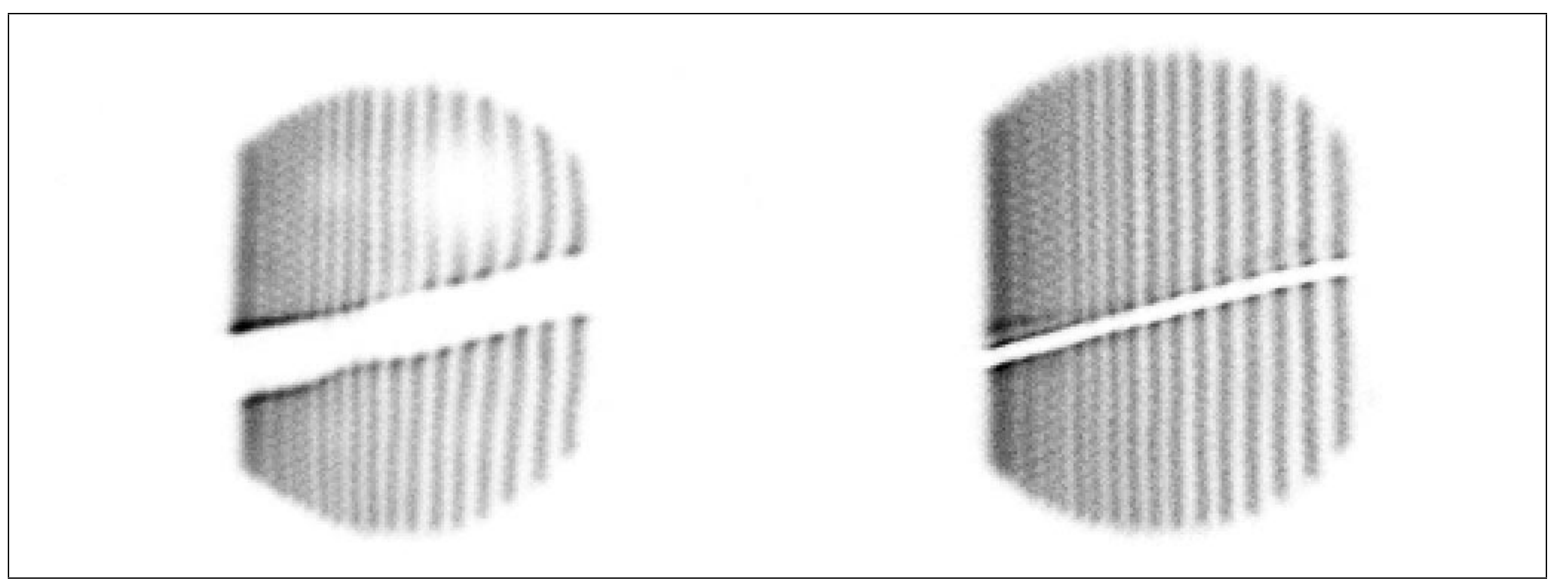

Abbildung 3.32: Szintigramm eines Linienphantoms, aufgenommen mit einem für Schilddrüsenszintigramme optimierten Kollimator. 


\section{Statistik der Zählung mit einer Gammakamera}

\subsection{Einleitung}

In den vorhergehenden Kapiteln sind die geometrischen Eigenschaften der Abbildung mit einer Gammakamera zusammen mit Aspekten der Impulshöhendetektion Gegenstand der Untersuchung gewesen. In diesem Kapitel wird die Gammakamera in ihrer Eigenschaft als lokalisierender Zähler untersucht.

Wie im Abschnitt 2.2.1 diskutiert, ist der radioaktive Zerfall selbst ist ein stochastischer Prozeß, d.h., das Abzählen detektierter $\gamma$-Quanten liefert auch bei idealer Messung prinzipiell nur fehlerbehaftete Schätzungen. Zwei Aufgabestellungen, die miteinander verbunden sind, ergeben sich:

1. Es ist der beste Schätzer zu finden, d.h. derjenige Schätzer, der im Mittel die kleinste Abweichung vom tatsächlichen Wert ergibt.

2. Reale Zählvorrichtungen sind unvollkommen, d.h. es können Ereignisse unterschlagen, aber auch fälschlicherweise hinzugefügt werden. Diese Fehler sind dadurch zu korrigieren, daß ein Modell des Zählprozesses den Zusammenhang zwischen tatsächlicher und gemessener Rate herstellt.

Der erste Punkt ist für die der Radioaktivität allgemein zugrunde liegenden PoissonVerteilung gelöst: Bei idealer Zählung ist die Rate durch den Quotienten aus gemessener Anzahl und Meßdauer abzuschätzen. Es gibt für diese Verteilung prinzipiell keinen besseren Schätzer, es handelt sich um einen sog. vollständigen Schätzer.

Der zweite Punkt dagegen ist in Zusammenhang mit der Gammakamera bisher nur ungenügend behandelt. Deshalb wird in diesem Kapitel die Gammakamera in ihrer Ei- 
genschaft als realer Zähler diskutiert. Die Kamera besitzt eine nur begrenzte Zählkapazität, wobei die Begrenzung ihre Ursache in Totzeiten findet, während derer die Kamera keine weiteren Gammaquanten erfolgreich detektieren kann. Die Konsequenz daraus sind Zählverluste. Darüber hinaus stellt sich nunmehr die erste Frage neu: Wenn die zugrunde liegende Verteilung nicht die Poisson-Verteilung ist, muß über die Wahl des Schätzers neu nachgedacht werden.

Der Abschnitt 4.2 behandelt die herkömmlichen Modelle zum Zählverhalten, worauf im folgenden Abschnitt 4.3 ein neues Modell dargelegt wird, welches auf die Besonderheiten der Gammakamera eingeht. Im Abschnitt 4.4 wird daraus ein neuer Schätzer entwickelt, der im Abschnitt 4.5 anhand von Computersimulationen und Messungen getestet wird.

\subsection{Bisherige Verfahren}

\subsubsection{Theorie}

Unter einem Zähler soll im Weiteren eine Anordnung verstanden werden, die zeitlich aufeinanderfolgende Impulse zählt. Dabei muß zwischen der Anzahl aller Ereignisse, die bei idealer Zählung gezählt würden und im folgenden als „angebotene Ereignisse“ bezeichnet seien, und den tatsächlich gezählten Ereignissen - entsprechend als „gezählte Ereignisse" gekennzeichnet - unterschieden werden [18]. Charakteristisch für jeden Zähler ist eine Totzeit $\tau$ während derer er für nachfolgende angebotene Ereignisse unempfindlich ist. Da die Erscheinung der Totzeit, die kein Zeitpunkt, sondern vielmehr ein Zeitintervall ist, ist es äußert hilfreich, Zähler nicht als Zähler von Zeitpunkten - an denen Impulse auftreten bzw. beginnen - sondern als Zähler von Intervallen zwischen gezählten Ereignissen zu betrachten.

Man kann Zähler danach unterscheiden, ob ein angebotenes Ereignis oder ein gezähltes Ereignis den Beginn einer Totzeit markiert [13].

Typ 1: Markiert ein angebotenes Ereignis jeweils unabhängig von anderen angebotenen Ereignissen den Beginn einer Totzeit, so spricht man von einem paralysierbaren Zähler. In der Abb. 4.1 ist diese Gesetzmäßigkeit im oberen Teil skizziert. Ein angebotenes Ereignis wird nur dann gezählt, wenn es eine zeitliche Differenz größer $\tau$ zu dem vorhergehenden angebotenen Ereignis besitzt. Da dieses für alle angebotene Ereignisse gleichermaßen gilt, kann bei hoher angebotener Ereignisrate der Zustand eintreten, daß nur noch sehr we- 


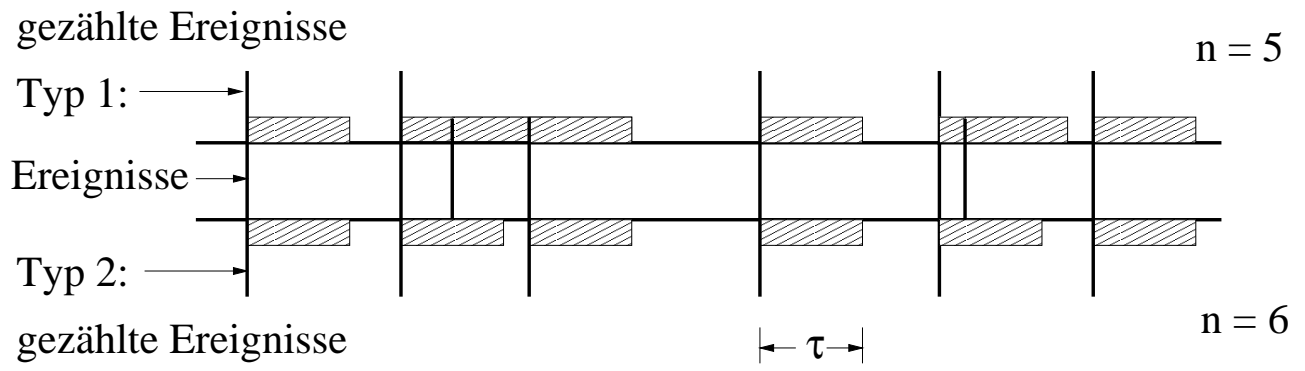

Abbildung 4.1: Paralysierbarer(1) und nicht-paralysierbarer Zähler(2). Die durchgezogenen senkrechten Linien kennzeichnen gezählte Ereignisse. Im oberen Teil definieren Linien im schraffierten Bereich den Beginn einer neuen Totzeit, ohne daß das angebotene Ereignis, welches diese Totzeit auslöst, gezählt wurde.

nige oder sogar überhaupt keine der angebotenen Ereignisse gezählt werden können, da die Intervalle der Länge $\tau$ überlappend den gesamten Meßzeitraum überdecken.

Typ 2: Kommt es zu einer Totzeit des Zählers nur aufgrund eines gezählten Ereignisses, so spricht man von einem nicht-paralysierbaren Zähler, weil mit steigender angebotener Ereignisrate die Zählrate $1 / \tau$ asymptotisch erreicht wird. Diesem Verhalten entspricht der untere Teil in Abb. 4.1.

Im Falle des radioaktiven Zerfalls (Poisson-Verteilung) kann man die Ausdrücke für die Größe der Zählverluste, die im Mittel auftreten, für die beiden Zählertypen leicht angeben.

Der Zähler des Typs 1 zählt genau die Intervalle, die durch angebotene Ereignisse begrenzt sind und größer als die Totzeit $\tau$ sind. Ist die angebotene Ereignisrate $\lambda$, so ist die Dichtefunktion $p(t)$ ihrer Intervallverteilung durch die Dichtefunktion der Exponentialverteilung,

$$
p(t)=\lambda e^{-\lambda t}
$$

gegeben. Daraus ergibt sich der Anteil $\alpha$ der Intervalle, die größer als $\tau$ sind zu

$$
\alpha=\int_{\tau}^{\infty} \lambda e^{-\lambda t} d t=e^{-\lambda \tau},
$$

und somit der Zusammenhang zwischen der Zahl $n$ der gezählten Ereignisse und der Zahl $N$ der angebotenen Ereignisse zu

$$
n=N \alpha=N e^{-\lambda \tau}
$$


In der Abb. 4.2 ist der Zusammenhang bzgl. der angebotenen Rate dargestellt. Die Anzahl der angebotenen Ereignisse und die Anzahl der gezählten Intervalle sind hier nur deshalb so einfach aufeinander zu beziehen, weil sowohl die Intervallängen voneinander unabhängige Zufallsgrößen sind als auch einer Intervallänge größer als $\tau$ genau ein registriertes Ereignis entspricht. Die Unabhängigkeit der Zufallsgrößen wird an dieser Stelle betont, weil in dem Fall, wo z.B. mehrere Intervalle an die Bedingung geknüpft sind, daß ein angebotenes Ereignis registriert wird, der Zusammenhang der Anzahl von registrierten Ereignissen und der Anzahl der angebotenen Intervalle komplizierter ist. Dieses kann z.B. auf die Gammakamera in ihrer Eigenschaft als Zähler zutreffen und wird in Abschnitt 4.3 besprochen.

Abbildung 4.2:

Verlauf gemessener zu tatsächlicher Zählrate bei paralysierbarer und nichtparalysierbarer Charakteristik.

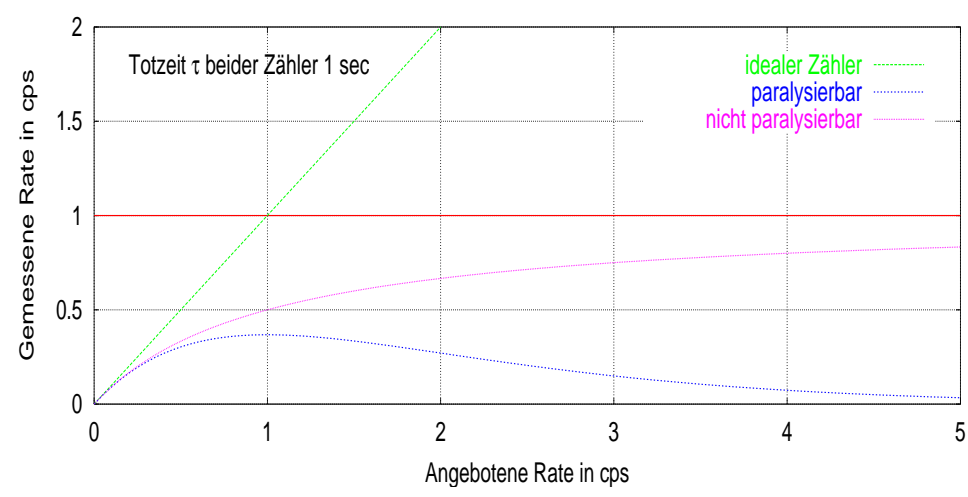

Bei dem Zähler des Typs 2 (nicht-paralysierbar), bei dem jeweils ein registriertes Ereignis eine Totzeit der Länge $\tau$ anstößt, ist das Verhältnis der Anzahl von angebotenen Ereignissen zu gezählten Ereignissen durch das Verhältnis der gesamten Meßdauer $T$ zu dem Anteil der Meßdauer gegeben, während derer die Apparatur empfindlich für Ereignisse ist. Sind $n$ Ereignisse registriert worden, so ist der Zähler eine Zeit $n \cdot \tau$ unempfindlich gewesen. Man erhält also

$$
N=\frac{n}{1-\frac{n}{T} \tau} .
$$

Der Verlauf in Bezug auf die Rate ist ebenfalls in Abb. 4.2 dargestellt.

\subsubsection{Anwendung auf die Gammakamera}

Es ist eine bekannte Tatsache, daß das Totzeitverhalten der Gammakamera weder mit dem Modell eines paralysierbaren, noch mit dem eines nicht-paralysierbaren Zählers über den gesamten Ratenbereich beschrieben werden kann [14, 20, 2, 34]. 
Zunächst ist zu präzisieren, wie die Größen $\dot{n}$ und $\dot{N}$ in diesem Rahmen definiert sind. Im allgemeinen versteht man unter $\dot{n}$ die gemessene Rate in einem gegebenen Impulshöhenfenster und dazugehörend $\dot{N}$ als tatsächliche Rate bzgl. dieses Fensters.

Beschränkt man sich auf den Bereich relativ kleiner Raten, so scheint in diesem Bereich der nicht-paralysierbare Zähler das Verhalten der Gammakamera am ehesten zu beschreiben. Bei hohen Raten allerdings zeigt sich ein paralysierbares Verhalten.

Um dennoch das Zählverhalten so weit wie möglich zu beschreiben, wurde bisher die Totzeit $\tau_{n}$ eines nicht-paralysierbaren Zählers nicht mehr als ratenunabhängig gesehen. Der Verlauf der Funktion $\tau_{n}(\dot{n})$ ist empirisch zu bestimmen [21]. $\tau_{n}(\dot{n})$ ist eine monoton steigende Funktion. Dabei hat sich gezeigt, daß eine solchermaßen definierte Totzeit von vielen Faktoren abhängig ist. Neben der gemessenen Rate $\dot{n}$ ist dabei vor allem die Breite $\Delta E$ des gewählten Impulshöhenfensters und der Anteil von Streustrahlung von Bedeutung $[5,41,1]$. Aus theoretischer Sicht ist eine solche, ohne weitere Begründung angenommene Abhängigkeit der Totzeit für einen nicht-paralysierbaren Zähler sehr unbefriedigend; ein Impulshöhenfenster kann im Listmode sogar im Anschluß an eine Messung gewählt werden. Dies führt zu der paradoxen Situation unterschiedlicher Totzeiten für ein und dieselbe Kamera bei einer einzigen Messung.

Einen ersten Schritt in Richtung konstanter Totzeiten vollzog das Modell zweier hintereinander geschalteter Zähler von Sorenson [29]. Dabei wird der erste Zähler als paralysierbar (Kristall, Pulshöhenanalysator), der zweite dagegen als nicht-paralysierbar (nachgeschaltete Elektronik) angenommen. Die Totzeit für den ersten Zähler sei $\tau_{p}$ und für den zweiten $\tau_{n}$. Gilt $\tau_{p} \geq \tau_{n}$ wird die Zählanordnung nachwievor allein durch (4.2) beschrieben. Gilt jedoch $\tau_{n}>\tau_{p}$, dann kann der Zusammenhang zwischen gemessener und angebotener Rate näherungsweise durch Einsetzen von (4.1) in (4.3) erhalten werden:

$$
\dot{n} \approx \frac{\dot{N} e^{-\dot{N} \tau_{p}}}{1+\dot{N} e^{-\dot{N} \tau_{p}}\left(\tau_{n}-\tau_{p}\right)}
$$

Allerdings ist die Bedingung $\tau_{n}>\tau_{p}$ fast nie erfüllt; heute ist $\tau_{n}$ fast immer kleiner als die physikalisch gegebene - Zeit $\tau_{p}$. Außerdem ist die Gleichung (4.4) aus zwei Gründen eine Näherung: Zum einen sind die am zweiten Zähler eingehenden Impulse nicht mehr rein zufällig, weil sie einen zeitlichen Mindestabstand von $\tau_{p}$ zueinander besitzen und zum anderen ist die effektive Totzeit des nicht-paralysierbaren Zählers immer kleiner als $\tau_{p}-\tau_{n}$. Obwohl diese Beschreibung eine Verbesserung darstellt, bleibt die Abhängigkeit der Totzeiten $\tau_{n}$ und $\tau_{p}$ von den Randbedingungen bestehen.

Als erste haben Wicks und Blau in [37] auf die Bedeutung der gesamten angebotenen 
Rate von Quanten ohne jegliches Impulshöhenfenster auf die Totzeitverluste hingewiesen und das Modell von Sorensen diesbezüglich erweitert. $\dot{N}$ stellt im weiteren nun die gesamte angebotene Rate dar. Wenn $\alpha \dot{N}$ den Anteil derjenigen Quanten im Impulshöhenfenster darstellt, so ist (4.4) folgendermaßen zu modifizieren:

$$
\dot{n} \approx \frac{\alpha \dot{N} e^{-\dot{N} \tau_{p}}}{1+\alpha \dot{N} e^{-\dot{N} \tau_{p}}\left(\tau_{n}-\tau_{p}\right)}
$$

Wicks und Blau konnten mit Hilfe dieses Modells zeigen, daß die Totzeiten $\tau_{p}$ und $\tau_{n}$ als nahezu konstant angenommen werden können. In der Praxis ist das Sorensonsche Modell wie auch seine Erweiterung wenig zum Einsatz gekommen, welches an dem zusätzlichen experimentellen wie rechnerischen Aufwand liegt, zwei Totzeiten zu bestimmen.

Zusammenfassend kann man vor allem zwei Punkte aus diesen Betrachtungen festhalten. Zum einen ist für die Totzeitverluste der Gammakamera die Gesamtrate der szintillierenden Gammaquanten entscheidend und zum anderen sollte ein Modell mit einer oder mehreren, jedoch konstanten Totzeiten auskommen. Dieses ist der Anlaß, um nach einem geeigneteren Modell zu suchen [11]. Im nächsten Abschnitt wird ein Modell vorgestellt, welches vor allem auf dem Phänomen der Signalüberlagerung (Pile-up) beruht. Es vereint beide oben genannten Eigenschaften.

\subsection{Das Modell der Signalüberlagerungsverluste}

\subsubsection{Die Signalüberlagerung (engl:Pile-up)}

Unter einer Signalüberlagerung oder einfach Überlagerung (Pile-up) versteht man das Auftreten eines oder mehrerer angebotener Ereignisse während der Verarbeitung eines vorangehenden initialen Ereignisses [9]. Bestimmt man z.B. die Energie eines Gammaquants durch Integration des Gesamtsignals aller Photomultiplier, so ist als untere Grenze für die Dauer der Verarbeitung die Szintillationsdauer im Kristall anzusehen. So beträgt für einen $\mathrm{NaI}(\mathrm{Tl})$-Kristall die Zeit, die ein Signal braucht, um auf ein Zehntel zurückzufallen, $0.9 \mu \mathrm{s}$ [7]. In der Abb. 4.3 ist ein Beispiel für eine Signalüberlagerung, die sich aus zwei Ereignissen zusammensetzt, gegeben.

Tritt eine Signalüberlagerung auf, so hat dies im wesentlichen drei Konsequenzen:

1. Nur das erste angebotene Ereignis wird „gezählt“. Schon an dieser Stelle kommt es zu Zählverlusten. 
Abbildung 4.3:

Beispiel für eine Signalüberlagerung, gemessen an dem Ausgang eines Photomultipliers. Die Quelle befand sich zentral vor diesem Photomultiplier. Die beiden Ereignisse liegen in etwa $0.28 \mu$ sec auseinander.

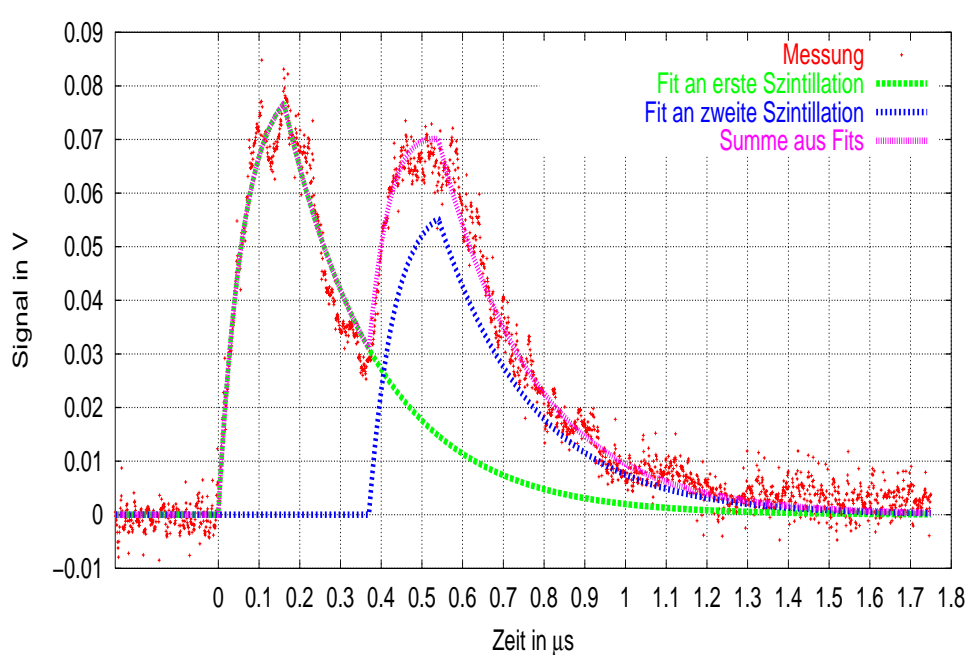

2. Falsche Ortszuweisung. Ebenso wie im Falle der Impulshöhenbestimmung ermittelt die Gammakamera in diesem Fall einen Ort, der einem gewichteten Mittel aus den der an der Signalüberlagerung beteiligten Ereignissen entspricht. Die Größe der Gewichte ist abhängig von den beteiligten Energien und den zeitlichen Abständen zum initialen Ereignis.

3. Falsche Bestimmung der Impulshöhe. Die Impulshöhe proportional zum Integral über die Summe der Signale aller Photomultiplier. Im Falle der Signalüberlagerung wird das erste Ereignis mit einem zusätzlichen Anteil in der Impulshöhe detektiert. Wie groß dieser Anteil ist, hängt einerseits von der Energie der folgenden Ereignisse und andererseits von deren zeitlichem Abstand zum initialen Ereignis ab.

Eine Signalüberlagerung beeinträchtigt alle Meßgrößen der szintigraphischen Abbildung und vermindert somit die Qualität u.U. erheblich. Punkt 2 in der obigen Auflistung führt in einem Szintigramm zu sog. Geisterbildern in dem Aktivität an Orten angezeigt wird, wo vielleicht gar keine ist. Punkt 3 führt dazu, daß bei Verwendung eines Impulshöhenfensters Ereignisse, deren Impulshöhe sich ohne Signalüberlagerung innerhalb des Fensters bewegt hätten, das Fenster verlassen und somit verworfen werden müssen. Andererseits werden z.B. gestreute Ereignisse, deren Impulshöhe ohne Signalüberlagerung unterhalb des Fensters gelegen hätte, zum Bildaufbau benutzt und verschlechtern die Bildqualität.

Um den schädlichen Einfluß der Signalüberlagerung zu vermindern, ist es notwendig, alle an der Überlagerung beteiligten Ereignisse, also auch das initiale Ereignis, zu erkennen und zu verwerfen [39, 38, 22]. Ein solcher Mechanismus wird als Überlagerungsverwerfung 
(engl:pile-up rejection) bezeichnet. Im Falle der vorliegenden Picker CX 250 Kamera ist ein derartiger Mechanismus über eine Kurvenerkennung im Summensignal (siehe Abb. 4.3) verwirklicht.

\subsubsection{Das neue Modell}

Im folgenden wird ein Modell der Zählverluste vorgestellt, welches auf der Vorstellung beruht, daß ausschließlich alle Ereignisverluste ihre Ursache in der Verwerfung der Signalüberlagerungen haben. Durch diese Modellvorstellung entsteht zu den vorher diskutierten Modellen der wesentliche Unterschied, daß ein angebotenes Ereignis niemals allein, sondern immer nur gemeinsam mit mindestens einem anderen angebotenen Ereignis verworfen wird. Insgesamt sind es drei Bedingungen, auf denen dieses Modell beruht:

1. Die Ereignisse unterliegen selbst einem Poisson Prozeß, d.h. die Verteilung der Intervallängen zweier aufeinander folgender Ereignisse ist durch die Exponentialverteilung gegeben. Ist die Rate der Ereignisse $\lambda$, so ist die Dichtefunktion der Intervallverteilung durch

$$
d p(t)=\lambda e^{-\lambda t} d t
$$

gegeben.

2. Der Mechanismus zur Erkennung einer Signalüberlagerung funktioniert perfekt. Es gibt neben den Verlusten, die durch Überlagerung entstehen, keine weiteren Zählverluste. Alle an der Signalüberlagerung beteiligten Ereignisse werden verworfen.

3. Eine Signalüberlagerung hat immer eine feste Zeitdauer $\tau$, beginnend mit dem initialen, an der Überlagerung beteiligten Ereignis. Diese Zeitdauer $\tau$ wird als Totzeit des Systems betrachtet. Nach $\tau$ ist die Gammakamera wieder zur Registrierung folgender Ereignisse bereit.

Der Punkt 3 ähnelt zunächst der Totzeitbedingung eines nicht-paralysierbaren Zählers, weil angebotene Ereignisse während einer Signalverarbeitung keine weitere Totzeit anstoßen. Es sei schon an dieser Stelle darauf hingewiesen, daß der Zähler unseres Modells trotzdem paralysierbar ist.

Ausgangspunkt der Betrachtungen bildet die Verteilung der Längen der beobachteten Intervalle. Eine beobachtete Intervallänge kann entweder einer tatsächlichen entsprechen (Abb. 4.4 a), oder aber zusammengesetzt sein aus mehreren tatsächlichen Intervallängen 
(Abb. 4.4 b-d), wobei die innerhalb des beobachteten Intervalls gelegenen Intervalle durch Signalüberlagerung maskiert sind. Der Zusammenhang zwischen der Dichtefunktion der

a

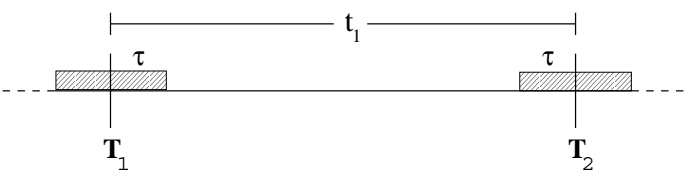

c

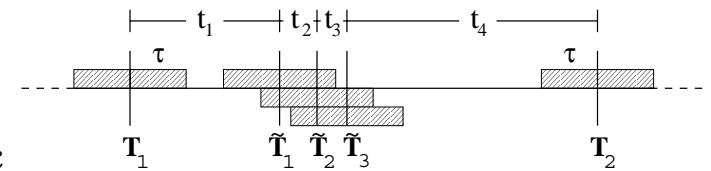

b

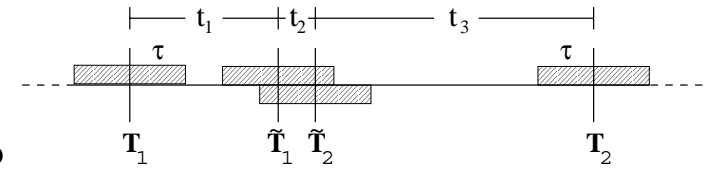

d

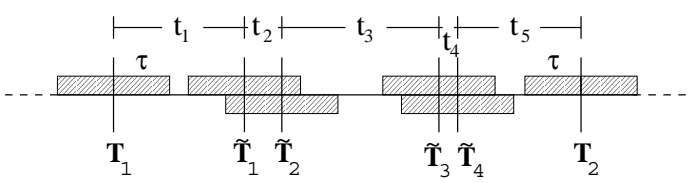

Abbildung 4.4: Intervalle mit bis zu vier verworfenen Ereignissen.

Intervalle der beobachteten Ereignisse und der angebotenen Ereignisse soll dadurch hergestellt werden, daß die Wahrscheinlichkeit, ein Intervall der Länge $t$ zu beobachten, entwickelt wird nach der Anzahl der verworfenen Ereignisse innerhalb dieses Intervalls. Dabei bedeutet ein Glied $d p_{n}(t)$ der Entwicklung die Wahrscheinlichkeit, das ein beobachtetes Intervall, dessen Länge zwischen $t$ und $t+d t$ liegt, $n$ durch Signalüberlagerung verworfene angebotene Ereignisse beinhaltet.

$$
d p(t)=\left\{\begin{array}{ll}
0 & t \leq \tau \\
d p_{0}(t)+d p_{1}(t)+d p_{2}(t)+d p_{3}(t)+\cdots & t>\tau
\end{array} .\right.
$$

Intervallängen $<\tau$ können nach den Bedingungen des Modells nicht beobachtet werden. Die $d p_{i}(t)$ stellen alle Möglichkeiten zur Bildung einer beobachtbaren Intervallänge dar, es gilt also $\int \sum_{i=0}^{\infty} d p_{i}=1$. Somit handelt es sich bei $d p(t)$ um eine normierte Wahrscheinlichkeitsdichte.

Der Term $d p_{0}$ entspricht einer tatsächlichen Intervallänge ohne verworfene Ereignisse. Es hat keine Signalüberlagerung gegeben, dieser Fall entspricht Abb. 4.4 a. Die Dichtefunktion ist

$$
d p_{0}(t)=\lambda e^{-\lambda t} d t, \quad \tau<t
$$

Für alle beobachteten Intervallängen, die kleiner als $2 \tau$ sind gilt, daß sie nur angebotenen Intervallängen entsprechen. Eine Signalüberlagerung bzw. selbst ein einzelnes angebotenes Ereignis innerhalb eines solchen beobachteten Intervalls führte automatisch dazu, daß mindestens eines der das beobachtete Intervall markierende Ereignis ebenfalls durch 
Signalüberlagerung verworfen werden müßte und das Intervall mithin nicht beobachtet würde.

Da es innerhalb des Modells kein einzelnes verworfenes Ereignis geben kann, gibt es auch keinen Term $d p_{1}(t)$, der nicht identisch verschwindet:

$$
d p_{1}(t) \equiv 0
$$

Das nächste Glied der Entwicklung, $d p_{2}(t)$, ist in Abb. 4.4 b skizziert: Zwischen den beobachteten Ereignissen zu den Zeitpunkten $T_{1}$ und $T_{2}$ hat es eine Signalüberlagerung, bestehend aus zwei Ereignissen, gegeben. Das beobachtete Intervall besteht demnach aus drei aufeinanderfolgenden tatsächlichen Intervallen. Die Länge dieser Intervalle ist nicht beliebig; die Intervalle $t_{1}$ und $t_{3}$ müssen größer, das Intervall $t_{2}$ muß dagegen kleiner als $\tau$ sein und natürlich gilt $t=t_{1}+t_{2}+t_{3}$. Die Wahrscheinlichkeit für das Auftreten dreier, stochastisch unabhängiger Intervallängen ist das Produkt ihrer Einzelwahrscheinlichkeiten,

$$
\lambda e^{-\lambda t_{1}} \lambda e^{-\lambda t_{2}} \lambda e^{-\lambda t_{3}} d t_{1} d t_{2} d t_{3}=\lambda^{3} e^{-\lambda\left(t_{1}+t_{2}+t_{3}\right)} d t_{1} d t_{2} d t_{3}
$$

und unter Verwendung von $t_{3}=t-t_{1}-t_{2}$ wird daraus $\lambda^{3} e^{-\lambda t} d t_{2} d t_{1} d t$. Das Intervall $t_{2}$ kann irgendwo zwischen $T_{1}+\tau$ und $T_{2}-\tau$ liegen. Die Gesamtwahrscheinlichkeit $d p_{2}(t)$ ergibt sich aus der Summe

$$
d p_{2}(t)=\int_{\tau}^{t-\tau}\left(\int_{0}^{\min \left(\tau, t-t_{1}-\tau\right)} d p\left(t_{2}\right) d t_{2}\right) d p\left(t_{1}\right) d t_{1} d t
$$

über alle diese Möglichkeiten. Die Minimumfunktion für die obere Grenze der Integration über $d t_{2}$ berücksichtigt, daß sich das Intervall $t_{2}$ nicht immer bis $\tau$ erstrecken kann, und zwar immer dann nicht, wenn einerseits die beobachtete Pausenlänge $t<3 \tau$ ist oder andererseits die Signalüberlagerung nahe an $T_{2}$ liegt, genauer wenn $T_{2}-\tilde{T}_{2}<2 \tau$ ist. Betrachtet man diese Fälle separat, so kann man die Minimumfunktion auflösen und erhält für $2 \tau<t<3 \tau$

$$
d p_{2}(t)=\lambda^{3} e^{-\lambda t} \int_{\tau}^{t-\tau} \int_{0}^{t-t_{1}-\tau} d t_{2} d t_{1} d t=\lambda^{3} e^{-\lambda t} \frac{1}{2}(t-2 \tau)^{2} d t
$$

und für $3 \tau<t$

$$
\begin{aligned}
d p_{2}(t) & =\lambda^{3} e^{-\lambda t}\left(\int_{\tau}^{t-2 \tau} \int_{0}^{\tau} d t_{2} d t_{1}+\int_{t-2 \tau}^{t-\tau} \int_{0}^{t-t_{1}-\tau} d t_{2} d t_{1}\right) d t \\
& =\lambda^{3} e^{-\lambda t} \frac{1}{2} \tau(2 t-5 \tau) d t .
\end{aligned}
$$


Die Funktion $d p_{2}(t)$ ist an der Stelle $t=2 \tau$ und $t=3 \tau$ stetig, wie leicht durch Einsetzen geprüft werden kann.

Die Wahrscheinlichkeiten $d p_{i}$ mit $i \geq 3$ lassen sich prinzipiell ebenso berechnen, allerdings nimmt die Komplexität der zu beachtenden Randbedingungen sehr schnell zu. Der Fall c in Abb. 4.4 mit $i=3$ umfaßt beispielsweise schon vier Intervalle, deren Bedingungen

$$
\text { 1. } t_{1}>\tau, \text { 2. } t_{2}+t_{3} \leq \tau, \text { 3. } t_{4}>\tau
$$

lauten und mit Hilfe der Minimumfunktion umgesetzt werden können,

$$
d p_{3}(t)=\lambda^{4} e^{-\lambda t} \int_{\tau}^{t-\tau} \int_{0}^{\min \left(\tau, t-t_{1}-\tau\right)} \int_{0}^{\min \left(\tau-t_{2}, t-t_{1}-t_{2}-\tau\right)} d t_{3} d t_{2} d t_{1} d t .
$$

Da drei maskierte Ereignisse nur eine einzige Signalüberlagerung darstellen können, kann die Minimumfunktion auf gleiche Weise aufgelöst werden, wie für Glg. (4.11). Es folgt für $2 \tau<t \leq 3 \tau$

$$
\begin{aligned}
d p_{3}(t) & =\lambda^{4} e^{-\lambda t} \int_{\tau}^{t-\tau} \int_{0}^{t-t_{1}-\tau} \int_{0}^{t-t_{1}-t_{2}-\tau} d t_{3} d t_{2} d t_{1} d t \\
& =\frac{1}{6} \lambda^{4} e^{-\lambda t}(t-2 \tau)^{3} d t
\end{aligned}
$$

und für $3 \tau<t$

$$
\begin{aligned}
d p_{3}(t) & =\lambda^{4} e^{-\lambda t}\left(\int_{\tau}^{t-2 \tau} \int_{0}^{\tau} \int_{0}^{\tau-t_{2}} d t_{3} d t_{2} d t_{1}+\int_{t-2 \tau}^{t-\tau} \int_{0}^{t-t_{1}-\tau} \int_{0}^{t-t_{1}-t_{2}-\tau} d t_{3} d t_{2} d t_{1}\right) d t \\
& =\frac{1}{6} \lambda^{4} e^{-\lambda t} \tau^{2}(3 t-8 \tau) d t .
\end{aligned}
$$

Allgemein kann für die Wahrscheinlichkeit einer einzelnen Signalüberlagerung, die aus $n>2$ Ereignissen besteht, aus dem Vorangegangenen

$$
d p_{(n)}(t)= \begin{cases}0 & t \leq 2 \tau \\ \frac{1}{n !} \lambda^{n+1}(t-2 \tau)^{n} e^{-\lambda t} d t & 2 \tau<t \leq 3 \tau \\ \frac{1}{n !} \lambda^{n+1} \tau^{n-1}(n t-(3 n-1) \tau) e^{-\lambda t} d t & 3 \tau<t\end{cases}
$$

geschlossen werden. Die Notation $d p_{(n)}(t)$ wird im nun folgenden Absatz erklärt.

Für den Fall von vier verworfenen Ereignissen sind die zu treffenden Fallunterscheidungen noch komplizierter. Neben der Möglichkeit, daß alle verworfenen Ereignisse eine einzelne Signalüberlagerung bilden, gibt es auch noch den Fall zweier getrennter,aus zwei 
Ereignissen bestehenden Überlagerungen, wie in Abb. 4.4 d dargestellt. Natürlich kann dieser Fall nur eintreten für eine beobachtete Pausenlänge von $t>3 \tau$. Um diese beiden Typen zu unterscheiden, wird folgende Notation eingeführt: Jede Zahl in einem von runden Klammern umgebenen Index zeigt eine Signalüberlagerung an, wobei die Zahl selbst die Anzahl der diese Überlagerung konstituierenden Ereignisse angibt. Für den Fall von vier verworfenen Ereignissen ergibt sich also

$$
d p_{4}(t)=d p_{(4)}(t)+d p_{(2,2)}(t)
$$

und für fünf oder sechs verworfenen Ereignisse muß man

$$
\begin{aligned}
& d p_{5}(t)=d p_{(5)}(t)+\underbrace{d p_{(2,3)}(t)+d p_{(3,2)}(t)}_{=2 d p_{(2,3)}(t)} \\
& d p_{6}(t)=d p_{(6)}(t)+2 d p_{(2,4)}(t)+d p_{(3,3)}(t),
\end{aligned}
$$

schreiben.

Bei der Berechnung von $d p_{(2,2)}(t)$ muß beachtet werden, daß die Intervallängen $t_{1}, t_{2}+t_{3}$ und $t_{5}$ größer, $t_{2}$ und $t_{4}$ dagegen kleiner als $\tau$ sein müssen:

$$
\begin{aligned}
d p_{(2,2)}(t) & =\lambda^{4} e^{-\lambda t} \int_{\tau}^{t-2 \tau} \int_{0}^{A} \int_{\tau-t_{2}}^{t-t_{1}-t_{2}-\tau} \int_{0}^{B} d t_{4} d t_{3} d t_{2} d t_{1} d t \\
A & =\min \left(\tau, t-t_{1}-\tau\right) \\
B & =\min \left(\tau, t-t_{1}-t_{2}-t_{3}-\tau\right) .
\end{aligned}
$$

Die Auflösung der Bedingungen für die Integrationsgrenzen führt zu einer ganzen Reihe von Fallunterscheidungen. Aus diesem Grund wird auf die Darstellung des exakten Ergebnisses für $d p_{(2,2)}(t)$, welches z.B. mit Hilfe eines Computer-Algebra Systems erhalten werden kann, verzichtet und statt dessen eine Abschätzung angegeben. Der Ausdruck der Abschätzung kann erhalten werden, indem die Minimumfunktionen in den Intervallgrenzen nicht ausgewertet, sondern immer zu $\tau$ gesetzt werden:

$$
d p_{4}(t) \leq \frac{1}{2} \lambda^{5} \tau^{2}(t-3 \tau)^{2} e^{-\lambda t}
$$

Um das Gewicht der Beiträge der einzelnen Ereignisse, die durch $d p_{i}(t)$ beschrieben werden, einschätzen zu können, müssen die Wahrscheinlichkeiten $P_{i}=\int^{\infty} p_{i}(t) d t$ berechnet werden:

$$
P_{0}=\int_{\tau}^{\infty} d p_{0}(t)=e^{-\lambda \tau}
$$




$$
\begin{aligned}
& P_{2}=\int_{2 \tau}^{\infty} d p_{2}(t)=e^{-2 \lambda \tau}\left(1-e^{-\lambda \tau}\right) \\
& P_{3}=\int_{2 \tau}^{\infty} d p_{3}(t)=e^{-2 \lambda \tau}\left(1-(1+\lambda \tau) e^{-\lambda \tau}\right) \\
& P_{4}=\int_{3 \tau}^{\infty} d p_{4}(t) \leq \frac{1}{2} \lambda^{2} \tau^{2} e^{-3 \lambda \tau}
\end{aligned}
$$

Um einen Eindruck der Größenordungen, um die es sich für die $P_{i}$ handelt, zu erhalten, sind in Tabelle 4.1 die Werte bis $i=4$ aufgetragen und zwar für die Situation einer angebotenen Rate von $10^{4}$ cps und Totzeiten von 1, 1.5, 3 und $10 \mu$ sec. Die sechste Spalte

\begin{tabular}{c||c|c|c|c|c}
$\lambda=10^{4} \mathrm{cps}$ & $P_{0}$ & $P_{2}$ & $P_{3}$ & $P_{4}$ & $\sum_{i>3}^{\infty} P_{i}$ \\
\hline \hline$\tau=1 \mu \mathrm{s}$ & 0.9901 & 0.0098 & $4.868 \cdot 10^{-5}$ & $\leq 4.852 \cdot 10^{-5}$ & $1.483 \cdot 10^{-4}$ \\
\hline$\tau=1.5 \mu \mathrm{s}$ & 0.9851 & 0.0144 & $1.081 \cdot 10^{-4}$ & $\leq 1.075 \cdot 10^{-4}$ & $3.319 \cdot 10^{-4}$ \\
\hline$\tau=3 \mu \mathrm{s}$ & 0.9704 & 0.0278 & $4.154 \cdot 10^{-4}$ & $\leq 4.154 \cdot 10^{-4}$ & $1.306 \cdot 10^{-3}$ \\
\hline$\tau=10 \mu \mathrm{s}$ & 0.9048 & 0.0779 & $3.828 \cdot 10^{-3}$ & $\leq 3.831 \cdot 10^{-3}$ & $1.342 \cdot 10^{-2}$
\end{tabular}

Tabelle 4.1: Wahrscheinlichkeiten der Ereignisse $P_{1}$ bis $P_{4}$ bei unterschiedlichen Totzeiten einer Gammakamera. Die angebotene Rate beträgt immer $10^{4} \mathrm{cps}$. In der sechsten Spalte ist die Wahrscheinlichkeit aufgetragen, daß ein Intervall nicht durch die ersten drei Typen beschrieben wird. Zu beachten ist, daß die Anzahl der im Mittel verworfenen Ereignisse aus diesen Wahrscheinlichkeiten noch zu berechnen ist.

in der Tabelle 4.1 stellt die Summe der Wahrscheinlichkeiten der $P_{i}, i>3$ dar. Dies ist eine Aussage darüber, wie groß die Wahrscheinlichkeit ist, daß ein beliebiges gemessenes Intervall durch eine dieser Ereignistypen beschrieben wird. Im nächsten Abschnitt wird mit Hilfe der $d p_{i}(t)$ ein Schätzer für die angebotene Rate konstruiert, wobei aufgrund der komplizierten Form der $d p_{i}$ nur endlich viele berücksichtigt werden können. Insofern stellt die sechste Spalte in Tabelle 4.1 einen groben Anhaltspunkt dafür dar, wie gut der in dieser Weise konstruierte Schätzer sein kann.

\subsection{Der neue Schätzer}

Eine gebräuchliche Methode zur Schätzung von Parametern von Verteilungen ist die Maximum-Likelihood-Methode. Die Anwendung dieser Methode in dem hier behandelten Fall stößt auf Schwierigkeiten. Einerseits kann keine geschlossen Form für die $d p_{i}(t)$ in 
der Entwicklung von $d p(t)$ angegeben werden und andererseits sind auch die berechneten $d p_{i}(t)$ nur stückweise definiert.

Trotzdem sollen die Ergebnisse der Maximum-Likelihood-Methode für die ungestörten Verteilungen (Exponential- und Poissonverteilung) als Leitfaden verwendet werden. Der Maximum-Likelihood Schätzer für die Intervallverteilung (Exponentialverteilung) von $N$ Intervallen ist durch

$$
\hat{\lambda}=\frac{N}{\sum_{i=1}^{N} t_{i}}
$$

gegeben und stellt das Reziproke der mittleren Intervallänge $\bar{t}$ dar. Dabei muß beachtet werden, daß dieser Schätzer für eine vorgegebene Anzahl $N$ von beobachteten Intervallen gilt. Die Meßvorschrift im Rahmen der Szintigraphie lautet oft anders, hier wird meist eine feste Meßdauer $t_{M}$ vorgegeben. In diesem Fall liegt eine multivariate Verteilung vor, abhängig von der Anzahl während der beobachteten Ereignisse $N$ und der beobachteten Intervallängen $t_{i}$,

$$
d p\left(N, t_{1}, \ldots, t_{N}\right)=\lambda^{N} e^{-\lambda t_{M}} d t_{1} \ldots d t_{2} .
$$

Der Maximum-Likelihood-Schätzer für diese Verteilung ist

$$
\hat{\lambda}=\frac{N}{t_{M}}
$$

Dies stimmt mit dem Maximum-Likelihood Schätzer für eine Poisson-Verteilung überein. Die Verwendung des Schätzers aus Glg. (4.25) im Falle fester, vorgegebener Meßdauer führt zu einer leichten Überschätzung des Parameters $\lambda$. Zusätzlich hat der Schätzer aus Glg. (4.27) den Vorteil, daß die Kenntnis der einzelnen $t_{i}$ nicht notwendig ist, die einfache Zählung innerhalb von $t_{M}$ ist ausreichend.

Die beiden diskutieren Schätzer (4.25) und (4.27) wären für eine ideale Gammakamera mit Totzeit $\tau=0$ die Schätzer der Wahl, jenachdem, ob man eine vorgegebene Anzahl von Quanten oder eine vorgegebene Meßdauer lang mißt. Für eine reale Gammakamera mit Totzeit $\tau \neq 0$ sind beide nicht mehr anwendbar. Um möglichst nahe an der MaximumLikelihood-Methode zu bleiben, schätzt man die mittlere beobachtete Intervallänge $\bar{t}$ wie durch Glg. (4.25) vorgegeben,

$$
\bar{t}=\frac{1}{N} \sum_{i=1}^{N} t_{i},
$$

ab. Es ist zu betonen, daß die $t_{i}$ nicht mehr der Exponentialverteilung gehorchen. Mit Hilfe der in Abschnitt 4.3.2 gefundenen Dichtefunktion $d p(t)$ für die Verteilung der beobachteten 
Intervallängen ist es jedoch möglich, die mittlere beobachtete Intervallänge $\bar{t}$ als Funktion des Parameters $\lambda$ der zugrunde liegenden Exponentialverteilung zu berechnen:

$$
\begin{aligned}
\bar{t}(\lambda) & =\int_{\tau}^{\infty} t d p(t)=\int_{\tau}^{\infty} \sum_{i=0}^{\infty} t d p_{i}(t) \\
& \approx \int_{\tau}^{\infty} \sum_{i=0}^{4} t d p_{i}(t) \\
& =\frac{1}{\lambda}\left(1+2 \lambda \tau+2(\lambda \tau)^{2}-\frac{23}{3}(\lambda \tau)^{3}+\frac{85}{12}(\lambda \tau)^{4}+\mathcal{O}(\lambda \tau)^{5}\right)
\end{aligned}
$$

Die Verbindung wird hergestellt, indem Glg. (4.30) in (Glg. 4.28) eingesetzt wird. Gelöst wird die resultierende Glg. durch Entwicklung des Parameters $\lambda$ bis zur zweiten Ordnung in $\tau, \lambda=a+b \tau+c \tau^{2}$. Man erhält

$$
\hat{\lambda}=\frac{N}{\sum_{i} t_{i}}\left(1+\frac{2 N}{\sum_{i} t_{i}} \tau+\frac{6 N^{2}}{\left(\sum_{i} t_{i}\right)^{2}} \tau^{2}+\mathcal{O}\left(\tau^{3}\right)\right) .
$$

Leider ist es aufgrund der komplizierten Form der Dichtefunktion nicht leicht, einen Schätzer für die Vorgabe einer festen Meßzeit zu finden und so soll hier die Annahme gemacht werden, daß die Verwendung der Näherung $\sum_{i} t_{i} \approx t_{M}$ zulässig ist. Somit erhält der neue Schätzer die Form

$$
\hat{\lambda}=\frac{N}{t_{m}}\left(1+\frac{2 N}{t_{m}} \tau+\frac{6 N^{2}}{t_{m}^{2}} \tau^{2}\right) .
$$

Der Faktor

$$
\mu=1+\frac{2 N}{t_{m}} \tau+\frac{6 N^{2}}{t_{m}^{2}} \tau^{2}
$$

ist unabhängig von der Auswahl einer Untermenge der beobachteten Ereignisse. Auch die Raten innerhalb eines Impulshöhenfensters oder eines ausgewählten Bereichs des Detektionskristalls müssen mit dem Faktor (4.33) multipliziert werden. Beobachtet man z.B. innerhalb eines Impulshöhenfensters $n$ Ereignisse, so ist die Anzahl der tatsächlich einfallenden Ereignisse innerhalb des Fensters über

$$
\hat{n}=n\left(1+\frac{2 N}{t_{m}} \tau+\frac{6 N^{2}}{t_{m}^{2}} \tau^{2}\right)
$$

abzuschätzen. Nachwievor bedeutet $N$ die gesamte Anzahl von Quanten, die die Gammakamera detektiert hat. 


\subsection{Messungen und Simulationen}

Die tatsächlichen Zählraten experimentell zu bestimmen, ist nicht möglich. So ist neben der Computersimulation, die im Abschnitt 4.5.1 dargelegt wird, die Kamera selbst das eingesetzt Instrument, das oben vorgestellte Modell zu überprüfen.

Eine Möglichkeit, die Gültigkeit des Überlagerungsmodells zu testen trotz fehlender Kenntnis der angebotenen Ereignisse, stellt die relative zeitliche Veränderung von Zählraten, z.B. mit Hilfe von Abklingkurven, dar. Zwei unterschiedliche Meßreihen sind durchgeführt worden und werden in den beiden Abschnitten 4.5.2 und 4.5.3 vorgestellt.

\subsubsection{Computersimulation}

Der Schätzer (4.34) stellt nur eine Näherung dar; anhand einer Computersimulation der Signalüberlagerung kann getestet werden, bis zu welchen Werten von $\lambda \tau$ die Anwendung des Schätzers sinnvoll ist ${ }^{1}$.

Abbildung 4.5:

Simulation eines Zählers mit Signalüberlagerungsverhalten und einer Totzeit $\tau=1.5 \mu$ sec. $\hat{\lambda}$ bzw. $N / t_{M}$ stellen den Mittelwert über jeweils 20 Schätzungen dar.

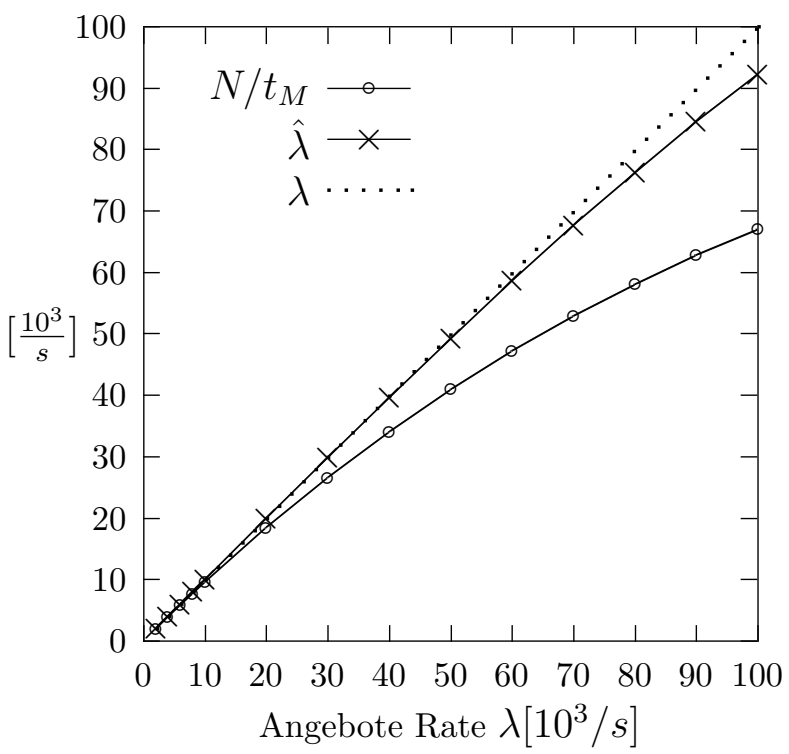

In der Abbildung 4.5 ist das Verhalten sowohl des Schätzers $N / t_{M}$ als auch des neuen Schätzers für verschiedene angebotene Zählraten aufgetragen. Simuliert wurde dabei eine Meßdauer von 60 Sekunden für jeden Wert der angebotenen Zählrate und einer Totzeit von $1.5 \mu$ sec. Jede „Messung“ wird zwanzigmal wiederholt, und der Mittelwert der jeweiligen

${ }^{1}$ Das Verhalten des neuen Schätzers hängt nur vom Produkt $\lambda \tau$ ab, für gleiche Werte von $\lambda \tau$ ergeben sich gleiche Zählratenverluste, auch bei unterschiedlichen Totzeiten $\tau$ 
Abbildung 4.6:

Abhängigkeit der Schätzung innerhalb einer Untermenge von der Gesamtrate. 60 Sekunden Meßdauer und 100 Meßwiederholungen. Auf der x-Achse ist die Gesamtrate aufgetragen.

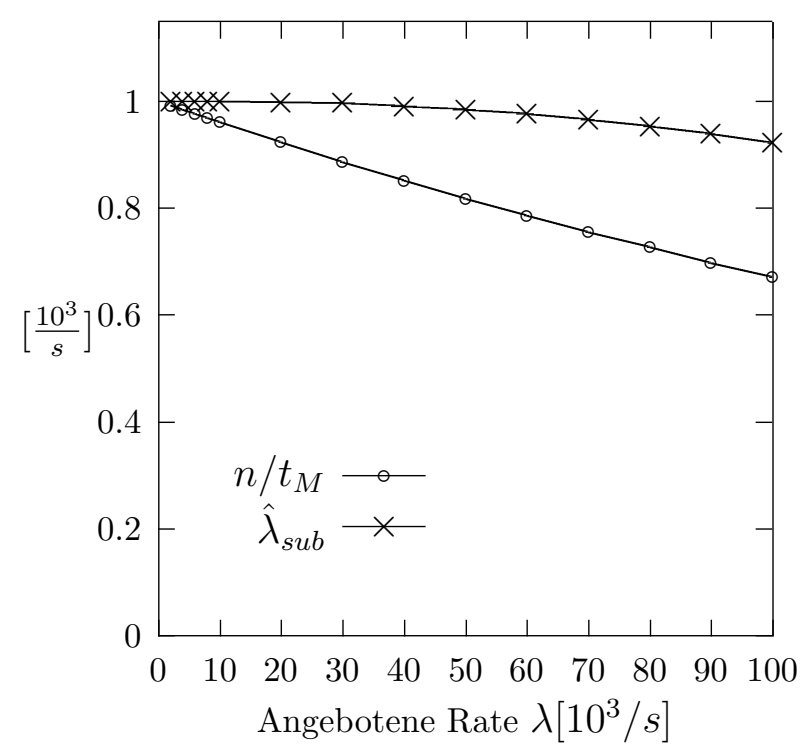

Schätzungen ist aufgetragen. Während der Schätzer $N / t_{M}$ nur bis zu Zählraten von 20.000 cps $(\lambda \tau=0.03)$ anwendbar ist, ist der neue Schätzer noch bis zu Zählraten von 50.000 $\operatorname{cps}(\lambda \tau=0.075)$ sehr gut.

Das Verhalten der Schätzer bzgl. einer Untermenge von Gammaquanten - definiert z.B. durch ein Impulshöhenfenster - in Abhängigkeit von der Gesamtrate kann ebenfalls simuliert werden. Angenommen wurden eine angebotene Rate von 1000 cps in der Untermenge, eine Meßdauer von 60 Sekunden und 100 Meßwiederholungen. Die Abbildung 4.6 zeigt die hohe Abhängigkeit des Maximum-Likelihood Schätzers $n / t_{M}$ von der Gesamtrate, wohingegen der neue Schätzer für eine konstant gute Schätzung über fast den Gesamten simulierten Bereich sorgt (Bis zu 40.000 cps ist die Abweichung geringer als $1 \%)$.

\subsubsection{Abklingkurve von ${ }^{99 m} \mathrm{Tc}$}

Wie eingangs dieses Kapitels erwähnt, kann die relative Änderung der Zählraten zur Überprüfung des Überlagerungsmodells benutzt werden, z.B. über die Bestimmung der Abklingkurve von ${ }^{99 m} \mathrm{Tc}$, beginnend in einem relativ hohen Zählratenbereich, ca $2 \cdot 10^{5}$ cps. Durch die Ratenverluste zu Beginn des Abklingens ist der Verlauf der Abklingkurve nach dem alten Maximum-Likelihood Schätzers flacher als der theoretisch zu erwartende; am Ende des Abklingens sind die Ratenverluste vernachlässigbar. Der neue Schätzer sollte den theoretisch zu erwartenden Abfall der Rate besser wiedergeben. Um den Erfolg zu 

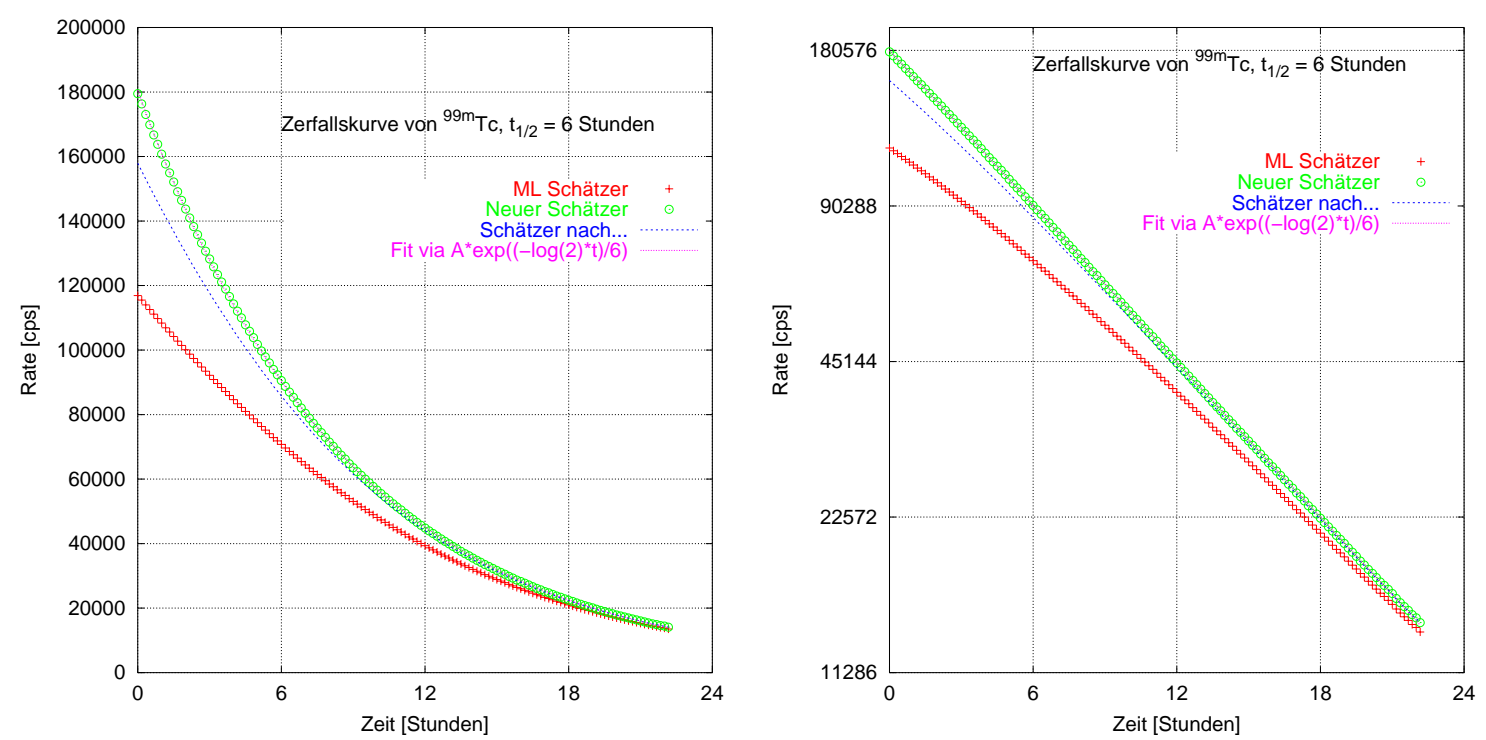

Abbildung 4.7: Vergleich zwischen Maximum-Likelihood Schätzer $n / t$ (rot) und neuem Schätzer (grün). Die blaue Kurve zeigt einen Fit an die Kurve des neuen Schätzers mit der Funktion $f(x)=A \cdot e^{-\frac{\ln (2)}{6.03} t}$, mit $A$ als einzigem Fitparameter. Der linke Plot zeigt lineare, der rechte halblogarithmische Auftragung.

unterstreichen, wird eine Funktion $f(t)=A \exp -\frac{\ln (2)}{t_{1 / 2}} \cdot t$ mit festem $t_{1 / 2}=6.03 h$ über den Parameter $A$ gefittet werden. In Abb.4.7 ist das Ergebnis einer Messung der Abklingkurve aufgetragen. Gemessen wurde alle 10 Minuten jeweils eine Minute lang. Die rote Kurve den Verlauf der Rate [cps], wie sie durch den Maximum-Likelihood Schätzer wiedergegeben wird, der sich aus dem Quotienten der innerhalb der 60 Sekunden gemessenen Anzahl von Gammaquanten und der Meßdauer ergibt.

\subsection{3 ${ }^{57}$ Co Punktquelle neben einer initial hochaktiven ${ }^{99 m}$ Tc Punktquelle}

In Anlehnung an die Computersimulation einer Untermenge von Gammaquanten soll hier der Versuch der gleichzeitigen Bestimmung der Raten zweier unterschiedlicher Punktstrahler mit stark unterschiedlichen Halbwertszeiten. Als Strahler werden ${ }^{99 m}$ Tc und ${ }^{57} \mathrm{Co}$ verwendet. Zur Identifikation der Gammaquanten kann neben der unterschiedlichen Zerfallsenergie die Lokalisation auf dem Gammakamerakopf selbst herangezogen werden. Laut Modell sind auch die Einzelraten der jeweiligen Strahler abhängig von der Gesamtrate, so daß auch eine relativ schwache ${ }^{57}$ Co Quelle $(\approx 1400[c p s])$ hohen relativen Ratenverlusten 
Abbildung 4.8:

Abklingkurve von ${ }^{57} \mathrm{Co}$ in $\mathrm{Ge}-$ genwart einer ${ }^{99 m} \mathrm{Tc}$-Quelle. In der unteren Abbildung ist der schnelle Abfall des ${ }^{99 m}$ Tc aufgetragen. Zunächst unterliegt das Abzählen der ${ }^{57}$ Co Quanten Verlusten durch Signalüberlagerung.
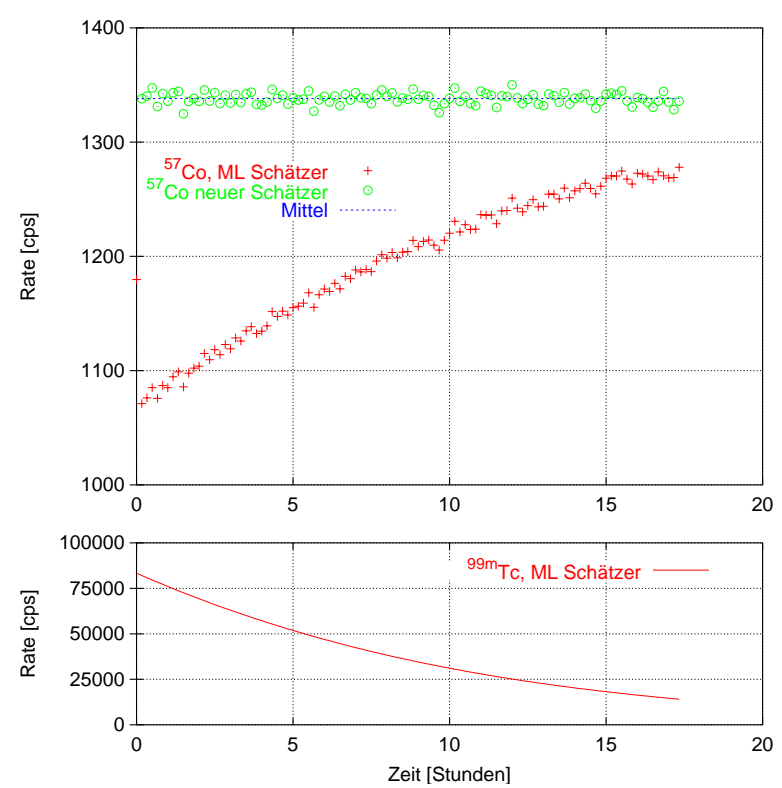

unterliegen muß, wie das zunächst mit einer hohen Ausgangsrate verwendete ${ }^{99 m} \mathrm{Tc}$. Aufgrund der wesentlich längeren Halbwertszeit des ${ }^{57}$ Co sollte in dem Maße, wie die Rate des ${ }^{99 m}$ Tc gegen Null geht, die Rate des ${ }^{57}$ Co sich asymptotisch der eigenen Abklingkurve nähern. Diese Abklingkurve, die sich in dem Zeitraum der Messung näherungsweise als eine Gerade darstellt, sollte sich ebenfalls durch die neue Schätzung zu den unterschiedlichen Zeitpunkten ergeben. Ein Meßergebnis mit Hilfe einer ${ }^{57}$ Co Punktquelle ist in Abb.4.8 dargestellt. 


\section{Schlußbemerkungen und Ausblick}

Im ersten Teil der vorliegenden Arbeit ist ein neues Verfahren zur Korrektion der Abbildung mit einer Gammakamera entwickelt worden, daß auf einer großen Zahl sequentiell aufgenommener Bilder eines nahezu monoenergetischen Feinnadelstrahls aus $\gamma$-Quanten beruht, der die Detektorfläche senkrecht und an wohldefinierten Orten durchquert. Man kann davon ausgehen, daß die Szintillationsorte der überwiegende Anzahl von $\gamma$-Quanten, die eine Szintillation im Kristall vorrufen, in dem von dem Feinnadelstrahl vorgegebenen Volumen liegen. Insofern ist die Antwort der Gammakamera auf diese Szintillationen spezifisch für die lokalen Abbildungseigenschaften die mit diesem Detektorareal verbunden sind.

Der Feinnadelstrahl wurde mit einer für diese Aufgabe erstellten und optimierten Feinnadelstrahlquelle erzeugt. Die Orte, an denen die Feinnadelstrahlquelle sequentiell positioniert wurde, um die jeweilige Messung durchzuführen, sind in einem regelmäßigen Raster angeordnet und werden als Rasterpunkte bezeichnet. Jede Messung an einem Rasterpunkt wurde einzeln analysiert, indem aus den Meßwerten, die zu jedem einzelnen $\gamma$-Quant gehören, charakteristische Größen wie z.B. der Mittelwert gebildet wurden. Diese charakteristischen Größen wurden dazu verwendet, um eine der lokalen Abbildungseigenschaften der individuellen Kamera angepaßte Diskretisierung des Szintigramms in Bildelemente zu definieren. Die Mittelwerte der Ortskoordinaten stellen die Mittelpunkte derjenigen Fläche dar, die zu einem Bildelement des Szintigramms gehört. Diese Fläche ist nicht mit der (quadratischen) Fläche des Bildelements des Szintigramms zu verwechseln, sondern ist die Fläche eines Polygons innerhalb der Fläche der möglichen Ortskoordinaten, die einem $\gamma$-Quant als Meßwert zugeordnet werden können. Diese Fläche zeichnet sich dadurch aus, daß die in ihr gelegenen Punkte den geringsten euklidischen Abstand zu dem zugeordneten Mittelwert aufweisen.

Neben der lokalisierenden Funktion ordnet die Gammakamera jedem detektierten Quant eine Impulshöhe zu, die bei idealen Bedingungen als Maß für die Energie des Quants ange- 
sehen werden kann. Durch die genaue Kenntnis der Position des von dem Feinnadelstrahl durchstrahlten Volumens, konnten die Eigenschaften der Impulshöhenzuweisung hoch aufgelöst betrachtet werden. Es stellte sich heraus, daß es dadurch möglich ist, diejenigen Impulshöhen, die zu Quanten gehören, die einer oder mehrerer Streuungen unterliegen, herauszufiltern, da diese mit einem verschobenen Ort detektiert wurden. So ist es in bisher unerreichter Weise möglich, die mittlere Impulshöhe derjenigen Quanten zu bestimmen, deren Wechselwirkung mit dem Kristall der Photoeffekt war. Da in der Szintigraphie zur Verwerfung compton-gestreuter Quanten ein Impulshöhenfenster eingesetzt wird, ist die Lage des Photopeaks von entscheidender Bedeutung für die Bildqualität.

Da jede Messung an einem Rasterpunkt nur eine Stichprobe aus der Meßwertverteilung darstellt, ist der Mindenstumfang einer Stichprobe pro Rasterpunkt bestimmt worden um den Fehler der Schätzung der Mittelwerte der Messungen nach oben abschätzen zu können.

Die entwickelte Korrektionsvorschrift ist mit Erfolg bei der Erstellung von Szintigrammen von Testmustern eingesetzt worden. Dabei ist eine Gammakamera zum Einsatz gekommen, deren Zustand weit vom Sollzustand entfernt liegt. Die erreichte Linearität der Abbildung darf dennoch als hervorragend bezeichnet werden. Die Korrektion der Impulshöhendetektion war ebenso erfolgreich, so daß das übliche $\pm 10 \%$-Impulshöhenfenster um den Photopeak herum ohne erkennbaren Nachteil eingesetzt werden konnte, obwohl dies bei dem vorliegenden Zustand der Gammakamera alles andere als selbstverständlich ist.

Die lokal aufgelöste Analyse der Meßwerte, die zu den Rasterpunkten gehören, ließe noch verfeinerte Korrektionsvorschriften zu, als die hier entwickelten, die nur mit den Mittelwerten der Stichproben arbeiten. Die Einbeziehung höherer statistischer Momente der Stichproben könnte insbesondere bei der Impulshöhe zu weiteren Verbesserungen führen. Die dargestellte Realisation der Feinnadelstrahlquelle ist ebenfalls nicht die einzig mögliche. Eine Konzeption mit einem Festköper als Strahlungsquelle statt einer Lösung, ist nicht nur sicherer im Umgang, sondern bietet z.B. mit Gadolinium einen Strahler ähnlicher Energie, jedoch weitaus längerer Halbwertszeit. Darüberhinaus kann dieser Strahler mit einer erheblich höheren spezifischen Aktivität hergestellt werden, so daß die langen Meßzeiten zur Aufnahme des Rasters zu einer akzeptablen Zeit zusammenschrumpfen.

Im zweiten Teil wurde die Statistik der Zählung von $\gamma$-Quanten mit einer Gammakamera unter besonderer Berücksichtigung der konkreten Detektionsmechanismen untersucht. Gab es bisher zwei Modelle zum Verhalten der Gammakamera bei hohen und niedrigen 
Zählraten die auch noch abhängig von den jeweiligen Raten und etwaigen angelegten Ortsund Impulshöhenfenstern waren, so konnte ein Modell gefunden werden, daß prinzipiell alle Zählratenbereiche beschreibt. Der Kernpunkt des Modells ist die Berücksichtigung des Signalüberlagerungseffekts, der die Verwerfung aller an der Signalüberlagerung beteiligten Quanten nötig macht. Ausgangspunkt der Betrachtungen stellt die Berechnung der Wahrscheinlichkeit dar, ein Zeitintervall $\delta$ zwischen erfolgreich (überlagerungsfrei) detektierten und damit zur Zählung gelangten Quanten zu beobachten. Die Berechnung der Wahrscheinlichkeit gelang durch eine Reihenentwicklung, wobei die Ordnung der Reihenglieder durch die Anzahl der in dem Zeitintervall liegenden verworfenen Gammaquanten bestimmt ist. Mit Hilfe dieser Wahrscheinlichkeitsverteilung wurde ein neuer Schätzer entwickelt, der aus der Anzahl der gezählten $\gamma$-Quanten auf die Anzahl der ohne Signalüberlagerung gezählten Quanten schließt. Dieser Schätzer ist mit Hilfe von Computersimulationen und Tests an der realen Gammakamera getestet worden.

Der neue Schätzer kann vor allem in Situationen mit Gewinn eingesetzt werden, wo quantitative Auswertungen hoher Qualität gewünscht sind, wie z.B. in kardiologischen Fragestellungen. 
Anhang 



\section{A Zur Simulation}

\section{A.1 Bestimmung der Brechungswinkel}

Das Zentrum der Szintillation habe die Eindringtiefe $z_{e}$ im Kristall. Bei gegebenem Abstand $r$ des Schnittpunkts des Strahls vom Lot des Szintillationszentrums auf die Ebene der Photokathoden sollen die Brechungswinkel $\alpha$ und $\beta$ zwischen dem Lichtleiter der Dicke $d_{L}$ und dem Kristall der Dicke $d_{K}$ bestimmt werden. $r^{\prime}$ sei der Abstand des Lotes des Szintillationszentrums vom Ort der Brechung, siehe auch Abb A.1.

Abbildung A.1:

Zur Brechung

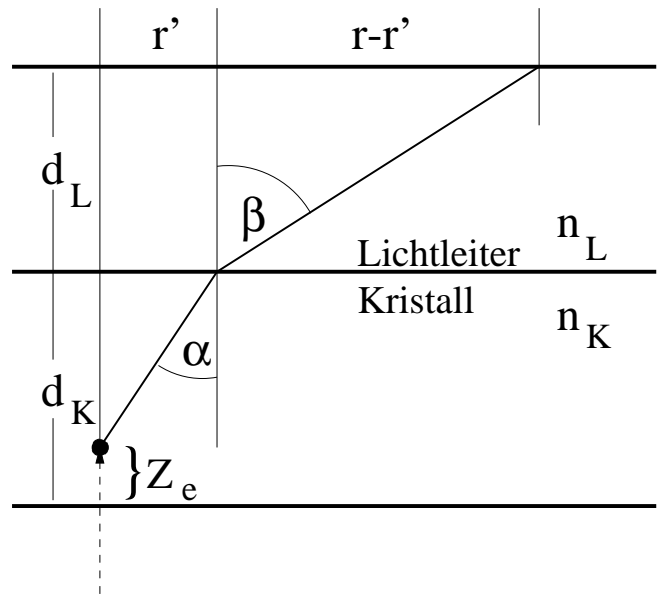

Zunächst ergeben sich die Brechungswinkel aus den Beziehungen

$$
\begin{aligned}
\sin \alpha & =\frac{r^{\prime}}{\sqrt{r^{\prime 2}+\left(d_{K}-z_{e}\right)^{2}}}, \\
\sin \beta & =\frac{r-r^{\prime}}{\sqrt{\left(r-r^{\prime}\right)^{2}+d_{L}^{2}}} .
\end{aligned}
$$


Das Brechungsgesetz

$$
n_{K} \sin \alpha=n_{L} \sin \beta
$$

ergibt mit A.1 die numerisch nach $r^{\prime}$ zu lösende Gleichung

$$
\frac{n_{K} r^{\prime}}{\sqrt{r^{\prime 2}+\left(d_{K}-z_{e}\right)^{2}}}-\frac{n_{L}\left(r-r^{\prime}\right)}{\sqrt{\left(r-r^{\prime}\right)^{2}+d_{L}^{2}}}=0 .
$$

Nach Berechnung von $r^{\prime}$ sind alle weiteren Größen bestimmt.

\section{A.2 Berücksichtigung von Reflektionen}

Das Szintillationslicht kann durch Reflektion an der Trennfläche zwischen Szintillationskristall und Lichtleiter einerseits und durch Spiegelung an der verspiegelten Seite des Kristalls andererseits mehrfach hin- und hergeworfen werden (siehe Abb. 2.8). Diese Beiträge zur Intensität des Szintillationslichts auf der Photokathode der Photomultiplier können berücksichtigt werden, indem jeder Spiegelung ein virtuelles Szintillationszentrum zugewiesen wird. Die virtuellen Szintillationszentren liegen entlang der Senkrechten des Kristalls die durch das reelle Szintillationszentrum führt. Demnach ist die Berechnung der reflektierten Beiträge analog zur Berechnung des direkten Anteils unter zu Hilfenahme eines virtuell fortgesetzten Kristalls. Lediglich die Lage der virtuellen Zentren ist zu berechnen.

Die gespiegelten Anteile spalten sich in zwei Stränge auf. Der erste Strang beginnt mit der Spiegelung an der verspiegelten Seite des Kristalls, der zweite Strang nimmt seinen Anfang mit der Reflektion an der Trennfläche zwischen Lichtleiter und Kristall. Jedes virtuelle Szintillationszentrum läßt sich über die Anzahl der Spiegelungen und Reflektionen eindeutig charakterisieren, also durch ein Zahlenpaar $\left(n_{s}, n_{r}\right)$. Die erste Zahl bedeutet die Anzahl der beteiligten Spiegelungen an der Aluminiumfolie, die zweite Zahl kennzeichnet die Anzahl der beteiligten Reflektionen an der Trennfläche Kristall/Lichtleiter.

Für den ersten Strang treten die folgenden Zahlenpaare auf: $(1,0),(2,1),(3,2)$ usw., beim zweiten sind es die Paare $(1,1),(2,2),(3,3)$ usw.. Der Abstand $A_{\left(n_{s}, n_{r}\right)}$ eines mit Hilfe dieser Paare gekennzeichneten virtuellen Szintillationszentrums $V S Z_{\left(n_{s}, n_{r}\right)}$ zur Trennfläche Kristall/Lichtleiter ergibt sich zu

$$
A_{\left(n_{s}, n_{r}\right)}=\left(n_{r}+2\right) d_{K}+(-1)^{\left(n_{r}+n_{s}+1\right)} z_{e}
$$


Die Länge des Lichtweges durch den Kristall ergibt sich nach Berechnung des Einfallswinkels $\alpha$ gemäß Abschnitt A.1 - dort ist der Abstand $d_{K}-z_{e}$ entsprechend durch $A_{\left(n_{s}, n_{r}\right)}$ $\mathrm{zu}$ ersetzen - zu

$$
L W_{\left(n_{s}, n_{r}\right)}^{K}=\frac{A_{\left(n_{s}, n_{r}\right)}}{\cos \alpha(r)}
$$

Die Länge des Lichtweges durch den Lichtleiter ist stets durch

$$
L W^{L}=\frac{d_{L}}{\cos \beta(r)}
$$

gegeben.

Die Intensität des Szintillationslichts auf dem Flächenelement $d F$ ist direkt proportional zum Eintrittswinkel $\beta$ und umgekehrt proportional zum Quadrat des Abstandes zum (virtuellen) Szintillationszentrums. Darüber hinaus ist die Güte $\eta_{S}$ der Verspiegelung und die Größe des Anteils $\eta_{R}(\alpha)$ bei Reflektion nach Fresnelscher Brechung zu berücksichtigen. Die Schwächung kann mittels Lambert-Beerschem Gesetz berechnet werden. Insgesamt ergibt sich somit

$$
d I(r)=\frac{\cos \beta(r)}{\left(L W^{(K)}+L W^{(L)}\right)^{2}} \eta_{S}^{n_{S}} \eta_{R}^{n_{R}} \exp \left(-\lambda_{K} L W^{(K)}-\lambda_{L} L W^{(L)}\right) d F
$$

Die folgende numerische Integration ergibt die Gesamtintensität des Szintillationslichtes. 


\section{Literaturverzeichnis}

[1] Hine G.J. Adams, R and Zimmerman C.D. Deadtime measurements in scintillation cameras under scatter conditions simulating quantitative nuclear cardiography. J.Nucl.Med., 19:pp.538, (1978).

[2] R. et al. Adams. Deadtime of scintillation camera system-definitions, measurement and applications. Med.Phys., 1((4)), (1974).

[3] P. Andreo. Monte carlo techniques in medical radiation physics. Phys.Med.Biol., 36(7):861-920, (1991).

[4] H. O. Anger. Scintillation camera. Rev.Sci.Instrum., 29:27-33, (1958).

[5] J.E. et al. Arnold. The influence of true counting rate and the photopeak fraction of detected events on anger camera deadtime. J.Nucl.Med., 15((6)):pp.412, (1974).

[6] R.G. Baker and D.H. Davis. Phys.Med.Biol., 12:51, (1967).

[7] J.B. Birks. The Theory and Practice of Scintillation Counting. Pergamon Press, first edition, (1964).

[8] I. et al. Buvat. Comparative assesment of nine scatter correction methods based on spectral analysis unsing monte carlo simulations. J.Nucl.Med., 36(8):1476-1488, (1995).

[9] Larsson I. Ceberg, C. and S. Strand. A new method for quantification of image distortion due to pile-up in scintillation cameras. Eur.J.Nucl.Med., 18:pp.959, (1991).

[10] W.K. Eby, F.S. und Jentschke. Fluorescence response on nai(tl) to nuclear radiations. Phys.Rev., 96, (1954). 
[11] Striker T. Engeland, U. and H. Luig. Count-rate statistics of the gamma camera. Phys.Med.Biol, 43:2939, (1998).

[12] D. Engelkemeir. Nonlinear response of nai(tl) to photons. Rev.Sci.Instr., 27:589, (1956).

[13] R.D. Evans. The atomic nucleus. McGraw Hill, (1955).

[14] G. S. Freedman, T. Kinsella, and A. Dwyer. A correction method for high-count-rate quantitative radionuclide angiography. Radiology, 104:713-715, (1972).

[15] J. Hohnerkamp. Stochastic Dynamical Systems: concepts, numerical methods, data analysis., volume 1, chapter 15, pages 489-508. VCH Publishers, (1994).

[16] A. Hughes and P.F. Sharp. Factors affecting gamma-camera non-uniformity. Phys.Med.Biol., 33(2):259-269, (1988).

[17] T.K. Johnson, C. Nelson, and D.L. Kirch. A new method for the correction of gamma camera nonuniformity due to spatial distortion. Phys. Med. Biol., 41:21792188, (1996).

[18] R. Jost. Bemerkungen zur mathematischen theorie der zähler. Helvetica Physica Acta, 20:173, (1947).

[19] S. Kirkpatrick. Simulated annealing. Journal of Statistical Physics, 34:975-986, (1984).

[20] Zasadny K.R. Ackerman R.J. Koral, K.F. J.Nucl.Med., 37:pp.78, (1996).

[21] R.C. Lange, G.S. Freedman, and S. Treves. Analysis of maximum frame rates for gamma-ray camera-computer systems. Phys.Med.Biol., 17(5):624-629, (1972).

[22] T.K. et al. Lewellen. Evaluation of a clinical scintillation camera with pulse tail extrapolation electronics. J.Nucl.Med., 30:pp.1554, (1989).

[23] Strand S.E. Ljungberg, M. A monte carlo program for the simulation of scintillation camera characteristics. Comput.Meth.Prog.Biomed., 29:257-272, (1989).

[24] K. Lüssenhop. Methodische untersuchung zur verbesserung der nuklearmedizinischszintigraphischen abbildung: Monte-carlo-simulationsrechung einer feinnadelstrahlquelle. Master's thesis, Universität Göttingen, (1997). 
[25] J.R. Mallard and M.J. Myers. Performance of a gamma camera. Phys.Med.Biol., 8:163-180, (1963).

[26] G. et al. Normand. Computer simulation of the light collection process in scintillation gamma-ray cameras. In Ediss C. McQuarie, S.A. and L.I. Wiebe, editors, Advances in Szintillation Counting, pages 176-189. University of Alberta,Canada.

[27] G. et al. Normand. Influence of optical and geometrical parameters on scintillation detection in gamma camera heads. In Ediss C. McQuarie, S.A. and L.I. Wiebe, editors, Advances in Szintillation Counting, pages 190-207. University of Alberta,Canada.

[28] T.N. Padikal, A.B. Ashare, and J.G. Kereiakes. Field flood uniformity correction: Benefits or pitfalls? J. Nucl. Med., 17(7):653-656, (1976).

[29] J.A. Sorenson. Deadtime characteristics of anger camera. J.Nucl.Med., 16(4):pp.284, (1975).

[30] S.S. Spector, V.A. Brookeman, C.D. Kylstra, and N.J. Diaz. Analysis and correction of spatial distortions produced by the gamma camera. J. Nucl. Med., 13(5):307-312, (1972).

[31] J. B. Svedberg. Image quality of a gamma camera system. Phys.Med.Biol., 13(4):597610, (1968).

[32] J. B. Svedberg. On the intrinsic resolution of a gamma camera system. Phys.Med.Biol., 17(4):514-524, (1972).

[33] M Tenhunen, J. Pyykkönen, M. Tenhunen-Eskelinen, K. Jaatinen, and J.T. Kuikka. Components of the flood-field uniformity index in a gamma camera. Phys. Med. Biol., 41:1209-1216, (1996).

[34] Hušák V. Ullmann, V. and L. Dubroka. Dead-time correction in dynamic radionuclide studies by computer. Eur.J.Nucl.Med., 3:pp.197, (1978).

[35] W. van Sciver. Alkali halide scintillators. IRE Trans.Nucl.Sci, NS-3(4):39, (1956).

[36] R. Wicks and M. Blau. Effect of spatial distortion on anger camera field-uniformity correction: Concise communication. J.Nucl.Med., 20:252-254, (1979). 
[37] R. Wicks and Blau.M. The effect of window fraction on the deadtime of anger cameras: Concise communication. J.Nucl.Med., 18(7):pp.732, (1977).

[38] Li H. Wong, W.H. and J. Uribe. A high count rate position decoding and energy measuring method for nuclear cameras using logic detectors. IEEE Transactions on Nuclear Science, 45(3):pp.1122, (1998).

[39] W.H. Wong and H. Li. A scintillation detector signal processing technique with active pileup prevention for extending scintillation count rates. IEEE Transactions on Nuclear Science, 45(3):pp.838, (1998).

[40] K.C. et al. Young. Reproducibility and action levels for gamma camera uniformity. Nucl.Med.Commun., 11:95-101, (1990).

[41] Koral K.F. Zasadny, K.R. and Swailem F.M. Dead time of an anger camera in dual-energy-window-acquisition mode. Med.Phys., $20((4))$, (1993). 


\section{Lebenslauf}

Daten Anschrift: Uwe Engeland

v.Ossietzky Straße 12

37083 Göttingen.

Familienstand: ledig.

Staatsangehörigkeit: deutsch.

12.November 1967

Geboren als Sohn des Goldschmieds Günther Engeland und Friedel Engeland, geb. Schröder auf der Insel Föhr.

$1974-1978$

Einschulung in die Grundschule Wyk.

Sommer 1978

Eintritt in das staatliche Gymnasium der Insel Föhr.

Mai 1987

Abitur am Gymnasium Insel Föhr.

$1987-1995$

Sommer 1990

$1993-1995$

Februar 1995

$1995-1996$

Herbst 1995

Studium der Physik und Chemie, Universität Göttingen.

Vordiplom in Physik.

Diplomarbeit am Institut für theoretische Physik der Universität Göttingen. Thema der Diplomarbeit: Quantenmechanik in beschleunigten Bezugssystemen.

Abschluß Diplom-Hauptprüfung mit Note „Sehr gut“.

Einjährige Mitarbeit am MPI für biophysikalische Chemie in Nikolausberg, Göttingen. Die Arbeitsgruppe wird von Prof.Dr.Erwin Neher geleitet. Arbeitsgebiet: Computersimulation von intrazellulären Transportphänomenen.

Start einer Zusammenarbeit mit Prof.Dr.Suitbert Ertel am Institut für Psychologie der Universität Göttingen. Entwicklung einer neuen statistischen Methode der Faktorenanalyse mit Namen Varimin.

Ab Juli $1996 \quad$ Eintritt in die Forschungsgruppe medizinische Physik in der Nuklearmedizin Göttingen bei Prof.Dr.Dr.Heribert Luig. Beginn der Dissertation. Thema: Neue statistische Methoden zur Korrektur der Abbildungsfehler einer Gammakamera

September $1999 \quad$ Verleihung des Philips-Preises für medizinische Physik durch die Deutsche Gesellschaft für medizinische Physik (DGMP) anläßlicher der Jahrestagung der DGMP 1999 in Passau für einen Auszug aus der oben genannten Dissertation. Titel: „Statistik der Zählung mit einer Gammakamera.“

Januar $2001 \quad$ Promotion zum Dr.rer.nat. am Fachbereich für Physik der Universität Göttingen. 


\section{Danksagung}

Diese Arbeit wurde in der Forschungsgruppe Medizinische Physik, Zentrum Radiologie, Abteilung Nuklearmedizin des Universitätsklinikums Göttingen angefertigt. Dem Leiter dieser Abteilung, Professor Dr.Dr.H.Luig danke ich für die Ermöglichung dieser Arbeit und deren kontinuierliche Förderung.

Ich möchte mich herzlich bei Dr.Timothy Striker für die fruchtbare und schöne Zusammenarbeit bedanken, ohne die diese Arbeit nicht möglich gewesen wäre. Ebenso viel Dank schulde ich Jörg Bertram für seinen hohen persönlichen Einsatz beim Messen, Korrekturlesen, und einfach für sein Freundschaft.

Ein großes Dankeschön geht natürlich an Dr.Gernot Ebel, nicht nur für Mahnung und Ansporn, sondern auch für den Beistand in schwierigen Tagen und die Arbeit während der heißen Phase.

Natürlich geht auch ein Dank an die noch nicht genannten Mitglieder der Arbeitsgruppe medizinische Physik, Herrn Dipl.Phys Karlheinz Schmidt und Dr.Carten Engelmann.

Herrn Dr.Nils Schramm habe ich nicht nur kostbares Wolfram zu verdanken, sondern auch einen guten Schuß Mut nach der Tagung in Aachen.

Meiner Freundin Nina Pustowka danke ich für die Geduld, die sie im Zusammenhang mit dieser Arbeit nicht nur einmal aufbringen mußte.

Meiner Mutter Friedel Engeland danke ich herzlich für die Ermöglichung des Weges, den ich bis hier gegangen bin. Und natürlich für die viele Mühe mit mir während meiner Schulzeit. 